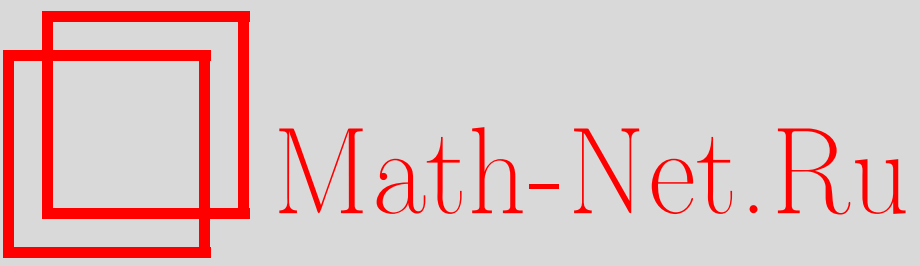

Н. Д. Копачевский, Е. В. Сёмкина, О малых движениях гидросистем, содержащих вязкоупругую жидкость, Итоги науки и техн. Сер. Соврем. мат. и ее прил. Темат. обз., 2019, том 172, 48-90

DOI: https://doi.org/10.36535/0233-6723-2019-172-48-90

Использование Общероссийского математического портала Math-Net.Ru подразумевает, что вы прочитали и согласны с пользовательским соглашением

http: //www.mathnet.ru/rus/agreement

Параметры загрузки:

IP: 34.229 .108 .108

26 апреля 2023 г., 14:02:00 


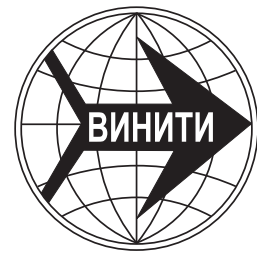

ИТОГИ НАУКИ И ТЕХНИКИ.

Современная математика и ее приложения.

Тематические обзоры.

Том 172 (2019). С. 48-90

DOI: $10.36535 / 0233-6723-2019-172-48-90$

УДК 517.958

\title{
О МАЛЫХ ДВИЖЕНИЯХ ГИДРОСИСТЕМ, СОДЕРЖАЩИХ ВЯЗКОУПРУГУЮ ЖИДКОСТЬ
}

\author{
(c) 2019 г. $\quad$ Н. Д. КОПАЧЕВСКИЙ, Е. В. СЕМКИНА
}

\begin{abstract}
АннотАция. Работа посвящена изучению двух задач, описывающих малые движения частично диссипативных гидросистем. Первая проблема касается малых движений гидросистемы, состоящей из вязкоупругой жидкости и баротропного газа, находящегося над жидкостью, вторая малых движений гидросистемы «вязкоупругая жидкость-идеальная жидкость-идеальная жидкость», заполняющих неподвижный сосуд. При помощи операторного подхода, разработанного в предыдущих работах авторов, обе задачи приведены к задаче Коши для дифференциально-операторного уравнения в некотором гильбертовом пространстве и доказана теорема о корректной разрешимости проблемы на произвольном конечном отрезке времени.
\end{abstract}

Ключевые слова: гидродинамическая система, идеальная жидкость, вязкоупругая жидкость, баротропный газ, ортопроектор, задача Коши.

\section{ON SMALL MOTIONS OF HYDRAULIC SYSTEMS CONTAINING A VISCOELASTIC FLUID}

\author{
(c) 2019 N. D. KOPACHEVSKY, E. V. SYOMKINA
}

\begin{abstract}
This paper is devoted to the study of two problems describing small motions of partially dissipative hydraulic systems. The first problem concerns small motions of a hydraulic system consisting of a viscoelastic fluid and a barotropic gas located above the fluid; the second concerns small motions of the hydraulic system "viscoelastic fluid-ideal fluid-ideal fluid" filling a static vessel. Using the operator approach developed in previous works of the authors, both problems are reduced to the Cauchy problem for a differential-operator equation in a Hilbert space and a theorem on the solvability of the problem on an arbitrary finite time interval is proved.
\end{abstract}

Keywords and phrases: hydrodynamic system, ideal fluid, viscoelastic fluid, barotropic gas, orthoprojector, Cauchy problem.

AMS Subject Classification: 76D05, 35Q30, 39A14, 39B42

Введение. Одними из первых работ, связанных с применением методов функционального анализа к исследованию проблемы малых движений и нормальных колебаний вязкоупругой жидкости в частично заполненном сосуде, являются работы А. И. Милославского (см. $[16-18,25,26])$. В них для обобщенной модели Олдройта $(m \geqslant 1)$ применен операторный подход, развивающий построения, проведенные ранее С. Г. Крейном и его учениками (см. $[3,13,14])$ применительно к задаче о малых колебаниях вязкой жидкости в частично заполненном сосуде. Исследования А. И. Милославского отражены, в частности, в монографии [23, гл. 8]. Случай полного заполнения сосуда вязкоупругой жидкостью рассмотрен в [2], а также в [23, п. 7.1]. Наконец, вариант, когда нижняя жидкость - обычная вязкая, а верхняя - идеальная сжимаемая жидкость, т.е. газ, изучен Б. М. Вронским и Н. Д. Копачевским (см. [4, 5,24$])$.

Работа выполнена при поддержке Российского научного фонда (проект № 16-11-10125. 
Данная работа посвящена исследованию операторными методами начально-краевых задач о малых движениях составных гидросистем, содержащих вязкоупругую жидкость. Так, в разделе 1 предполагается, что жидкость занимает в состоянии покоя нижнюю часть сосуда, а газ находится над ней. В процессе малых колебаний такой гидросистемы учитывается действие гравитационного поля с постоянным ускорением, а также малого поля внешних сил, наложенных на это поле.

Изложим кратко содержание раздела 1. В п. 1.1 дается математическая постановка начально-краевой задачи о малых движениях гидросистемы. Затем выводится закон баланса полной энергии гидросистемы для классического решения задачи. Это дает возможность в п. 1.2 осуществить выбор функциональных гильбертовых пространств и их подпространств, в которых естественно проводить исследование задачи. Далее в п. 1.3 проводится применение метода ортогонального проектирования на выбранные подпространства и изучение на его основе свойств операторов вспомогательных краевых задач, действующих в выбранных подпространствах. Затем в п. 1.4 осуществляется преобразование операторными методами уравнений движения вязкоупругой жидкости, а также кинематического условия на границе раздела «жидкость-газ» и уравнения движения газа. В п. 1.5 исходная начально-краевая задача переформулируется в векторно-матричной форме в виде задачи Коши для интегро-дифференциального уравнения первого порядка в ортогональной сумме пространств. При этом изучаются свойства возникших операторных матриц, являющихся операторными коэффициентами уравнения. Далее в п. 1.6 с использованием формы модели Олдройта вязкоупругой жидкости проводится дальнейшее видоизменение задачи и ее переформулировка в виде задачи Коши для дифференциально-операторного уравнения в расширенном гильбертовом пространстве. Выясняется, что основной оператор задачи (операторная матрица) является аккретивным и может быть расширен путем его замыкания до максимального аккретивного оператора. Это дает возможность в п. 1.7 использовать теорию сжимающих полугрупп операторов, действующих в гильбертовом пространстве, и на этой основе доказать теорему существования сильного (по времени) решения задачи Коши для интегро-дифференциального уравнения первого порядка, к исследованию которого была приведена исходная начально-краевая задача. В п. 1.8, являющимся последним в разделе, дана постановка задачи о нормальных (собственных) колебаниях гидросистемы и сформулирована соответствующая спектральная задача для оператор-функции (операторного пучка), обобщающей как известный пучок С. Г. Крейна (вязкая жидкость в частично заполненном сосуде), так и операторный пучок, отвечающий проблеме нормальных колебаний вязкоупругой жидкости в полностью заполненном сосуде.

По этой же схеме проводится изложение в разделе 2 , где изучается задача о малых движениях системы из трех тяжелых несмешивающихся однородных жидкостей, полностью заполняющих неподвижный сосуд. При этом нижняя жидкость считается вязкоупругой, а остальные две идеальными. Отметим, что случай, когда сосуд полностью заполнен системой из двух жидкостей, одна из которых является вязкоупругой, а другая идеальной, рассмотрен в [11], а вариант, когда неподвижный сосуд заполнен системой из двух вязкоупругих жидкостей, изучен в [9].

\section{1. О МАЛЫХ ДВИЖЕНИЯХ ГИДРОСИСТЕМЫ «ВЯЗКОУПРУГАЯ ЖИДКОСТЬ-БАРОТРОПНЫЙ ГАЗ»}

1.1. Математическая постановка задачи. Будем считать, что неподвижный сосуд $\Omega \subset \mathbb{R}^{3}$ заполнен двумя средами: вязкоупругой жидкостью (обобщенная модель Олдройта, см. [16, 20]) и баротропным газом. В этой модели связь между тензором вязких напряжений и удвоенным тензором скоростей деформаций в вязкоупругой жидкости описывается не простейшим законом Гука, а линейным дифференциальным соотношением, где фигурируют производные порядка $m \geqslant 1$ по времени как тензора вязких напряжений, так и тензора скоростей деформаций. Эта гидросистема находится в поле сил тяжести, и в состоянии покоя граница Г раздела жидкости и газа горизонтальна, т.е. расположена перпендикулярно действию гравитационного поля. При этом жидкость занимает область $\Omega_{1}$, ограниченную твердой стенкой $S_{1}$ и поверхностью $\Gamma$, а газ 


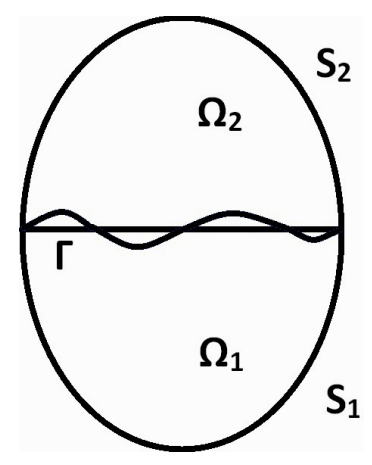

Рис. 1

находится выше жидкости и ограничен твердой стенкой $S_{2}$ и снизу равновесной поверхностью $\Gamma$ (см. рис. 1).

Выберем декартову систему координат $O x_{1} x_{2} x_{3}$ таким образом, чтобы ее начало было на $\Gamma$, а ось $O x_{3}$ была направлена вертикально вверх. Тогда ускорение силы тяжести есть $\boldsymbol{g}=-g \boldsymbol{e}_{3}$, $g>0$.

В состоянии покоя давление в жидкости $P_{1,0}(x)$ изменяется по закону Архимеда:

$$
P_{1,0}=P_{1,0}(0)-\rho_{1} g x_{3},
$$

где $\rho_{1}=$ const $>0$ - плотность жидкости, а $P_{1,0}(0)$ - давление на границе $Г$. Далее, газ называют баротропным, если его давление $P_{2}(t, x)$ связано с полем плотности $\tilde{\rho}_{2}$ соотношением

$$
P_{2}(t, x)=a^{2} \tilde{\rho}_{2}(t, x), \quad x=\left(x_{1}, x_{2}, x_{3}\right) \in \Omega_{2},
$$

$a^{2}$ - квадрат скорости звука. В состоянии покоя имеем связь

$$
P_{2,0}(x)=a^{2} \rho_{2,0}(x), \quad \nabla P_{2,0}=-g \rho_{2,0}(x) \boldsymbol{e}_{3},
$$

откуда следует, что

$$
\begin{gathered}
\rho_{2,0}=\rho_{2,0}\left(x_{3}\right)=\rho_{2,0}(0) \exp \left(-\frac{g x_{3}}{a^{2}}\right), \quad \rho_{2,0}(0)>0 \\
P_{2,0}=P_{2,0}\left(x_{3}\right)=P_{2,0}(0)+a^{2} \rho_{2,0}(0) \exp \left(-\frac{g x_{3}}{a^{2}}\right), \quad P_{2,0}=P_{1,0}(0) .
\end{gathered}
$$

Рассмотрим малые движения гидросистемы, близкие к состоянию покоя. В качестве искомых функций, описывающих движение системы, будем рассматривать: для жидкости - малое поле скоростей $\boldsymbol{u}(t, x), x \in \Omega_{1}$, и функцию вертикального отклонения границы раздела жидкости и газа $x_{3}=\zeta(t, \hat{x}), \hat{x}=\left(x_{1}, x_{2}\right) \in \Gamma$; для газа соответственно считаем искомыми отклонение $p_{2}(t, x)$ давления от равновесного $(1.1 .2),(1.1 .3)$, поле скоростей в газе $\boldsymbol{u}_{2}(t, x)$, а также отклонение $\rho_{2}(t, x)$ поля плотности от равновесного поля (1.1.3).

Заметим еще, что жидкость считаем вязкоупругой, подчиненной обобщенной модели Олдройта, когда напряжения в ней определяется не полем $\boldsymbol{u}_{1}(t, x)$, а полем

$$
\boldsymbol{v}_{1}(t, x)=\boldsymbol{u}_{1}(t, x)+\sum_{k=1}^{m} \alpha_{k} \int_{0}^{t} e^{-\beta_{k}(t-s)} \boldsymbol{u}_{1}(s, x) d s=: I_{01}(t) \boldsymbol{u}_{1},
$$

где $\alpha_{k} \geqslant 0, \beta_{k}>0, k=\overline{1, m}$ (см. [16,20]), - коэффициенты вязкоупругости. При $\alpha_{k}=0, k=\overline{1, m}$, получаем, в частности, модель обычной вязкой несжимаемой жидкости, а при $k=1$-модель Олдройта.

Выпишем теперь полную постановку начально-краевой задачи о малых движениях рассматриваемой гидросистемы.

Линеаризованные уравнения Навье-Стокса имеют вид

$$
\rho_{1} \frac{\partial \boldsymbol{u}_{1}}{\partial t}-\mu_{1} \Delta \boldsymbol{v}_{1}+\nabla p_{1}=\rho_{1} \boldsymbol{f}_{1}(t, x), \quad \operatorname{div} \boldsymbol{u}_{1}=0 \quad \text { в } \Omega_{1},
$$


где $\mu_{1}>0$ - коэффициент динамической вязкости, а $\boldsymbol{f}_{1}(t, x)$ - малое поле внешних сил, наложенное на гравитационное поле $\boldsymbol{g}=-\boldsymbol{g} \boldsymbol{e}_{3}$. Линеаризованные уравнения движения газа и уравнение неразрывности таковы:

$$
\begin{gathered}
\rho_{2,0} \frac{\partial \boldsymbol{u}_{2}}{\partial t}+\nabla p_{2}+\rho_{2} g \boldsymbol{e}_{3}=\rho_{2,0} \boldsymbol{f}_{2}, \quad p_{2}=a^{2} \rho_{2}, \\
\frac{\partial \rho_{2}}{\partial t}+\operatorname{div}\left(\rho_{2,0} \boldsymbol{u}_{2}\right)=0 \quad \text { в } \Omega_{2},
\end{gathered}
$$

где $\boldsymbol{f}_{2}(t, x)$ - заданное малое поле внешних сил в газе.

Выпишем теперь краевые условия в исследуемой проблеме. Для вязкой жидкости - это условия прилипания на твердой стенке:

$$
\boldsymbol{u}_{1}=\mathbf{0} \quad \text { на } S_{1}
$$

а для газа - условие непротекания:

$$
\boldsymbol{u}_{2} \cdot \boldsymbol{n}=0 \quad \text { на } S_{2},
$$

где $\boldsymbol{n}$ - внешняя нормаль к $S_{2}$. Далее, на границе раздела Г должны выполняться кинематические условия

а также динамические условия

$$
\boldsymbol{u}_{1} \cdot \boldsymbol{e}_{3}=\boldsymbol{u}_{2} \cdot \boldsymbol{e}_{3}=\frac{\partial \zeta}{\partial t}
$$

$$
\begin{gathered}
\mu_{1} \tau_{j 3}\left(\boldsymbol{v}_{1}\right)=0, \quad j=1,2, \quad \tau_{j k}\left(\boldsymbol{v}_{1}\right):=\frac{\partial v_{1, j}}{\partial x_{k}}+\frac{\partial v_{1, k}}{\partial x_{j}}, \\
{\left[-p_{1}+\mu_{1} \tau_{33}\left(\boldsymbol{v}_{1}\right)\right]-\left[-p_{2}\right]=-g\left(\rho_{1}-\rho_{2,0}(0)\right) \zeta .}
\end{gathered}
$$

Кроме того, должно выполняться линеаризованное условие сохранения объема жидкости:

$$
\int_{\Gamma} \zeta d \Gamma=0 .
$$

Наконец, в начальный момент времени $t=0$ следует задать начальные условия для полей скоростей, а также для отклонения границы раздела и поля давлений в газе:

$$
\begin{gathered}
\boldsymbol{u}_{k}(0, x)=\boldsymbol{u}_{k}^{0}(x), \quad x \in \Omega_{k}, \quad k=1,2, \\
p_{2}(0, x)=p_{2}^{0}(x), \quad x \in \Omega_{2}, \quad \zeta\left(0, x_{1}, x_{2}\right), \quad\left(x_{1}, x_{2}\right) \in \Gamma .
\end{gathered}
$$

Таким образом, начально-краевая задача о малых совместных движениях вязкоупругой жидкости и баротропного газа, заполняющих неподвижный сосуд $\Omega$, состоит в нахождении искомых функций из уравнений движения (1.1.5)-(1.1.7), краевых условий (1.1.8), (1.1.9), кинематических условий (1.1.10), динамических условий (1.1.11), условий сохранения объема (1.1.12) и начальных условий (1.1.13).

Будем считать, что задача (1.1.5)-(1.1.13) имеет классическое решение, т.е. все слагаемые в уравнениях, краевых и начальных условиях являются непрерывными функциями своих переменных. Для вывода закона баланса полной энергии гидросистемы воспользуемся следующими тождествами.

Первое тождество следует из (1.1.1)-(1.1.3):

$$
\nabla p_{2}+\rho_{2} g \boldsymbol{e}_{3}=a^{2} \rho_{2,0} \nabla\left(\rho_{2,0}^{-1} \rho_{2}\right)=\rho_{2,0} \nabla\left(\rho_{2,0}^{-1} p_{2}\right) .
$$

Второе тождество - это формула Грина для соленоидальных векторных полей, удовлетворяющих условию прилипания на твердой стенке:

$$
\begin{gathered}
\int_{\Omega_{1}} \boldsymbol{u}_{1} \cdot\left(-\mu_{1} \Delta \boldsymbol{v}_{1}+\nabla p_{1}\right) d \Omega_{1}=\mu_{1} E_{1}\left(\boldsymbol{u}_{1}, \boldsymbol{v}_{1}\right)-\int_{\Gamma}\left(\sum_{j, k=1}^{3} u_{1 j} \cos \left(\widehat{\boldsymbol{n}, \boldsymbol{e}_{k}}\right)\left[\tau_{j k}\left(\boldsymbol{v}_{1}\right)-p_{1} \delta_{j k}\right]\right) d \Gamma, \\
\operatorname{div} \boldsymbol{u}_{1}=\operatorname{div} \boldsymbol{v}_{1}=0 \quad \text { в } \Omega_{1}, \quad \boldsymbol{u}_{1}, \boldsymbol{v}_{1}=\mathbf{0} \quad \text { на } S_{1} ;
\end{gathered}
$$


здесь

$$
E_{1}\left(\boldsymbol{u}_{1}, \boldsymbol{u}_{1}\right):=\frac{1}{2} \int_{\Omega_{1}} \sum_{j, k=1}^{3}\left|\tau_{j k}\left(\boldsymbol{u}_{1}\right)\right|^{2} d \Omega_{1},
$$

а $E_{1}\left(\boldsymbol{u}_{1}, \boldsymbol{v}_{1}\right)$ - соответствующее полуторалинейное выражение. Величина $\mu_{1} E_{1}\left(\boldsymbol{u}_{1}, \boldsymbol{v}_{1}\right)$ равна скорости диссипации энергии в вязкоупругой жидкости, занимающей область $\Omega_{1}$.

Из уравнения (1.1.5) получаем соотношение

$$
\rho_{1} \int_{\Omega_{1}} \boldsymbol{u}_{1} \cdot \frac{\partial \boldsymbol{u}_{1}}{\partial t} d \Omega_{1}+\int_{\Omega_{1}} \boldsymbol{u}_{1} \cdot\left(-\mu_{1} \Delta \boldsymbol{v}_{1}+\nabla p_{1}\right) d \Omega_{1}=\rho_{1} \int_{\Omega_{1}} \boldsymbol{u}_{1} \cdot \boldsymbol{f}_{1} d \Omega_{1} .
$$

Из (1.1.6) с учетом (1.1.14) имеем

$$
\int_{\Omega_{2}} \rho_{2,0} \boldsymbol{u}_{2} \cdot \frac{\partial \boldsymbol{u}_{2}}{\partial t} d \Omega_{2}+\int_{\Omega_{2}} \boldsymbol{u}_{2} \cdot\left(\rho_{2,0} \nabla\left(\rho_{2,0}^{-1} p_{2}\right)\right) d \Omega_{2}=\int_{\Omega_{2}} \rho_{2,0} \boldsymbol{u}_{2} \cdot \boldsymbol{f}_{2} d \Omega_{2} .
$$

Второе слагаемое слева в (1.1.16) с учетом (1.1.15) и граничных условий (1.1.10), (1.1.11), а также уравнения (1.1.7), преобразуется следующим образом:

$$
\begin{gathered}
\int_{\Omega_{1}} \boldsymbol{u}_{1} \cdot\left(-\mu_{1} \Delta \boldsymbol{v}_{1}+\nabla p_{1}\right) d \Omega_{1}=\mu_{1} E_{1}\left(\boldsymbol{u}_{1}, \boldsymbol{v}_{1}\right)-\int_{\Gamma}\left(\sum_{j, k=1}^{3} u_{1 j} \cos \left(\widehat{\boldsymbol{n}, \boldsymbol{e}_{k}}\right)\left[\tau_{j k}\left(\boldsymbol{v}_{1}\right)-p_{1} \delta_{j k}\right]\right) d \Gamma= \\
=\mu_{1} E_{1}\left(\boldsymbol{u}_{1}, \boldsymbol{v}_{1}\right)-\int_{\Gamma} u_{1,3} p_{2} d \Gamma+g\left(\rho_{1}-\rho_{2,0}(0)\right) \int_{\Gamma}\left(\boldsymbol{u}_{1} \cdot \boldsymbol{e}_{3}\right) \zeta d \Gamma= \\
=\mu_{1} E_{1}\left(\boldsymbol{u}_{1}, \boldsymbol{v}_{1}\right)-\int_{\Gamma} u_{1,3} p_{2} d \Gamma+g\left(\rho_{1}-\rho_{2,0}(0)\right) \int_{\Gamma}\left(\boldsymbol{u}_{1} \cdot \boldsymbol{e}_{3}\right) \zeta d \Gamma= \\
=\mu_{1} E_{1}\left(\boldsymbol{u}_{1}, \boldsymbol{v}_{1}\right)-g\left(\rho_{1}-\rho_{2,0}(0)\right) \int_{\Gamma} \frac{\partial \zeta}{\partial t} \zeta d \Gamma+\int_{\Gamma}\left(\boldsymbol{u}_{1} \cdot \boldsymbol{n}\right) p_{2} d \Gamma= \\
=\mu_{1} E_{1}\left(\boldsymbol{u}_{1}, \boldsymbol{v}_{1}\right)-\frac{1}{2} g\left(\rho_{1}-\rho_{2,0}(0)\right) \frac{d}{d t} \int_{\Gamma}|\zeta|^{2} d \Gamma+\int_{\Gamma}\left(\boldsymbol{u}_{1} \cdot \boldsymbol{e}_{3}\right) p_{2} d \Gamma .
\end{gathered}
$$

Второе слагаемое слева в (1.1.17) преобразуется с учетом (1.1.7), (1.1.10) и связи $p_{2}=a^{2} \rho_{2}$ так:

$$
\begin{aligned}
& \int_{\Omega_{2}} \boldsymbol{u}_{2} \cdot\left(\rho_{2,0} \nabla\left(\rho_{2,0}^{-1} p_{2}\right)\right) d \Omega_{2}=\int_{\Omega_{2}} \operatorname{div}\left(p_{2} \boldsymbol{u}_{2}\right) d \Omega_{2}-\int_{\Omega_{2}} \rho_{2,0}^{-1} p_{2} \operatorname{div}\left(\rho_{2,0} \boldsymbol{u}_{2}\right) d \Omega_{2}= \\
& =-\int_{\Gamma} p_{2} \boldsymbol{u}_{2} \cdot \boldsymbol{e}_{3} d \Gamma+a^{-2} \int_{\Omega_{2}} \rho_{2,0}^{-1} p_{2} \frac{\partial p_{2}}{\partial t} d \Omega_{2}=-\int_{\Gamma} p_{2} \boldsymbol{u}_{2} \cdot \boldsymbol{e}_{1} d \Gamma+a^{-2} \frac{d}{d t} \int_{\Omega_{2}} \rho_{2,0}^{-1}\left|p_{2}\right|^{2} d \Omega_{2} .
\end{aligned}
$$

Складывая левые и правые части (1.1.16) и (1.1.17) и учитывая (1.1.18), (1.1.19), приходим к тождеству

$$
\begin{gathered}
\frac{1}{2} \frac{d}{d t}\left\{\rho_{1} \int_{\Omega_{1}}\left|\boldsymbol{u}_{1}\right|^{2} d \Omega_{1}+\int_{\Omega_{2}} \rho_{2,0}(x)\left|\boldsymbol{u}_{2}\right|^{2} d \Omega_{2}\right\}+ \\
+\frac{1}{2} \frac{d}{d t}\left\{a^{-2} \int_{\Omega_{2}} \rho_{2,0}^{-1}(x)\left|p_{2}\right|^{2} d \Omega_{2}+g\left(\rho_{1}-\rho_{2,0}(0)\right) \int_{\Gamma}|\zeta|^{2} d \Gamma\right\}= \\
\quad=-\mu_{1} E_{1}\left(\boldsymbol{u}_{1}, \boldsymbol{v}_{1}\right)+\rho_{1} \int_{\Omega_{1}} \boldsymbol{u}_{1} \cdot \boldsymbol{f}_{1} d \Omega_{1}+\int_{\Omega_{2}} \rho_{2,0} \boldsymbol{u}_{2} \cdot \boldsymbol{f}_{2} d \Omega_{2},
\end{gathered}
$$


которое является законом баланса полной энергии исследуемой гидросистемы, записанным в дифференциальной форме. Здесь слева в фигурных скобках стоит удвоенная кинетическая энергия (первое слагаемое), отвечающая полю скоростей в жидкости и газе, а второе слагаемое-это потенциальная энергия, отвечающая отклонению $\zeta$ границы раздела Г и изменению плотности в баротропном газе $\left(p_{2}=a^{2} \rho_{2}\right)$ в процессе малых колебаний. Справа в (1.1.20) стоит мощность сил, действующих на систему: это внутренние вязкоупругие силы (первое слагаемое) и внешние силы в областях $\Omega_{1}, \Omega_{2}$ (остальные слагаемые).

1.2. Выбор функциональных пространств. Из формулы (1.1.20) следует, что для описания движения гидросистемы следует привлечь к рассмотрению такие функциональные гильбертовы пространства, для которых поля скоростей и давлений должны приводить в любой момент времени к конечной кинетической и потенциальной энергиям системы, а также к конечной скорости диссипации энергии.

Перейдем к подробному рассмотрению этого вопроса и введем соответствующие пространства и их подпространства.

1.2.1. Прежде всего введем (комплексное) гильбертово пространство $\boldsymbol{L}_{2}\left(\Omega_{1}\right)$ векторных полей $\left\{\boldsymbol{u}_{1}(x)\right\}, x \in \Omega_{1}$, с квадратом нормы

$$
\left\|\boldsymbol{u}_{1}\right\|_{\boldsymbol{L}_{2}\left(\Omega_{1}\right)}^{2}:=\int_{\Omega_{1}}\left|\boldsymbol{u}_{1}(x)\right|^{2} d \Omega_{1}
$$

и соответствующим скалярным произведением. Как известно (см. [10,22]), пространство $\boldsymbol{L}_{2}\left(\Omega_{1}\right)$ имеет ортогональное разложение

$$
\begin{gathered}
\boldsymbol{L}_{2}\left(\Omega_{1}\right)=\boldsymbol{J}_{0}\left(\Omega_{1}\right) \oplus \boldsymbol{G}_{h, S_{1}}\left(\Omega_{1}\right) \oplus \boldsymbol{G}_{0, \Gamma}\left(\Omega_{1}\right), \\
\boldsymbol{J}_{0}\left(\Omega_{1}\right):=\left\{\boldsymbol{w}_{1} \in \boldsymbol{L}_{2}\left(\Omega_{2}\right): \operatorname{div} \boldsymbol{w}_{1}=0 \text { в } \Omega_{1}, \boldsymbol{w}_{1} \cdot \boldsymbol{n}_{1}=0 \text { на } \partial \Omega_{1}\right\}, \\
\boldsymbol{G}_{h, S_{1}}\left(\Omega_{1}\right):=\left\{\boldsymbol{v}_{1}=\nabla \Phi_{1} \in \boldsymbol{L}_{2}\left(\Omega_{1}\right): \Delta \Phi_{1}=0 \text { в } \Omega_{1}, \frac{\partial \Phi_{1}}{\partial n_{1}}=0 \text { на } S_{1}, \int_{\Gamma} \Phi_{1} d \Gamma=0\right\}, \\
\boldsymbol{G}_{0, \Gamma}\left(\Omega_{1}\right):=\left\{\nabla \varphi_{1} \in \boldsymbol{L}_{2}\left(\Omega_{1}\right): \varphi_{1}=0 \text { на } \Gamma\right\} .
\end{gathered}
$$

Здесь $\boldsymbol{n}_{1}$ - внешняя нормаль к $\partial \Omega_{1}, \boldsymbol{n}_{1}=\boldsymbol{e}_{3}$ на $\Gamma$, а операции $\operatorname{div}$ и $\partial / \partial n_{1}$ понимают в смысле обобщенных функций (распределений).

Будем считать, что части $S_{1}$ и $Г$ границы $\partial \Omega_{1}$ области $\Omega_{1}$ являются липшицевыми, причем контур $\partial S_{1}=\partial \Gamma$ также липшицев (см. $\left.[1,19]\right)$. Тогда для функций из

$$
H_{\Gamma}^{1}\left(\Omega_{1}\right):=\left\{\Phi(x) \in H^{1}\left(\Omega_{1}\right): \int_{\Gamma} \Phi d \Gamma=0\right\}, \quad\|\Phi\|_{H_{\Gamma}^{1}\left(\Omega_{1}\right)}^{2}:=\int_{\Omega_{1}}|\nabla \Phi|^{2} d \Omega_{1},
$$

имеет место обобщенная формула Грина для оператора Лапласа (см. [8]):

$$
(\Psi, \Phi)_{H_{\Gamma}^{1}\left(\Omega_{1}\right)}:=\int_{\Omega_{1}} \nabla \Psi \cdot \nabla \Phi d \Omega_{1}=\langle\Psi,-\Delta \Phi\rangle_{L_{2}\left(\Omega_{1}\right)}+\left\langle\gamma \Psi, \frac{\partial \Phi}{\partial n_{1}}\right\rangle_{L_{2, \Gamma}} \quad \forall \Psi, \Phi \in H_{\Gamma}^{1}\left(\Omega_{1}\right) .
$$

Здесь $L_{2, \Gamma}:=L_{2}(\Gamma) \ominus\left\{1_{\Gamma}\right\}-$ подпространство пространства $L_{2}(\Gamma)$, ортогональное единичной функции $1_{\Gamma}:=\left.1\right|_{\Gamma}$,

$$
\begin{gathered}
-\Delta \Phi \in\left(H_{\Gamma}^{1}\left(\Omega_{1}\right)\right)^{*}, \quad H_{\Gamma}^{1}\left(\Omega_{1}\right) \hookrightarrow L_{2}\left(\Omega_{1}\right) \hookrightarrow\left(H_{\Gamma}^{1}\left(\Omega_{1}\right)\right)^{*}, \\
\frac{\partial \Phi}{\partial n_{1}} \in\left(H_{\Gamma}^{1 / 2}\right)^{*}, \quad H_{\Gamma}^{1 / 2}:=H^{1 / 2}(\Gamma) \cap L_{2, \Gamma} \hookrightarrow L_{2, \Gamma} \hookrightarrow\left(H_{\Gamma}^{1 / 2}\right)^{*}, \quad \boldsymbol{n}_{1}=e_{3},
\end{gathered}
$$

$\gamma: \mathcal{L}\left(H_{\Gamma}\left(\Omega_{1}\right) ; H_{\Gamma}^{1 / 2}\right), \gamma \Psi:=\left.\Psi\right|_{\Gamma}$ - оператор следа на Г. Угловыми скобками в (1.2.5) обозначаются значения соответствующих функционалов, отвечающих оснащениям в (1.2.6). 
Отметим, что в силу постановки задачи (см. (1.1.5), (1.1.8), (1.1.10)) поле скоростей $\boldsymbol{u}_{1}(t, x)$ должно принадлежать при любом $t \geqslant 0$ подпространству $\boldsymbol{J}_{0}\left(\Omega_{1}\right) \oplus \boldsymbol{G}_{h, S_{1}}\left(\Omega_{1}\right)$, а поле градиентов давлений - подпространству $\boldsymbol{G}_{h, S_{1}}\left(\Omega_{1}\right) \oplus \boldsymbol{G}_{0, \Gamma}\left(\Omega_{1}\right)$.

1.2.2. Введем теперь пространство $\boldsymbol{L}_{2}\left(\Omega_{2} ; \rho_{2,0}\right)$ с квадратом нормы

$$
\left\|\boldsymbol{u}_{2}\right\|_{\boldsymbol{L}_{2}\left(\Omega_{2} ; \rho_{2,0}\right)}^{2}:=\int_{\Omega_{2}} \rho_{2,0}(x)\left|\boldsymbol{u}_{2}\right|^{2} d \Omega_{2},
$$

отвечающее полю скоростей в газе. Его ортогональное разложение, которое далее понадобится, таково:

$$
\boldsymbol{L}_{2}\left(\Omega_{2} ; \rho_{2,0}\right)=\boldsymbol{J}_{0}\left(\Omega_{2} ; \rho_{2,0}\right) \oplus \boldsymbol{G}\left(\Omega_{2} ; \rho_{2,0}\right),
$$

где

$$
\begin{aligned}
& \boldsymbol{J}_{0}\left(\Omega_{2} ; \rho_{2,0}\right):=\left\{\boldsymbol{v} \in \boldsymbol{L}_{2}\left(\Omega_{2} ; \rho_{2,0}\right): \operatorname{div}\left(\rho_{2,0} \boldsymbol{v}\right)=0 \text { в } \Omega_{2}, \rho_{2,0} \boldsymbol{v} \cdot \boldsymbol{n}_{2}=0 \text { на } \partial \Omega_{2}\right\}, \\
& \boldsymbol{G}\left(\Omega_{2} ; \rho_{2,0}\right):=\left\{\nabla \varphi_{2} \in \boldsymbol{L}_{2}\left(\Omega_{2} ; \rho_{2,0}\right): \int_{\Omega_{2}} \rho_{2,0} \varphi_{2} d \Omega_{2}=0\right\}=\boldsymbol{G}_{0, \Gamma}\left(\Omega_{2} ; \rho_{2,0}\right) \oplus \boldsymbol{G}_{h, S_{2}}\left(\Omega_{2} ; \rho_{2,0}\right), \\
& \boldsymbol{G}_{0, \Gamma}\left(\Omega_{2} ; \rho_{2,0}\right):=\left\{\nabla \psi_{2} \in \boldsymbol{L}_{2}\left(\Omega_{2} ; \rho_{2,0}\right): \psi_{2}=0 \text { на } \Gamma\right\}, \\
& \boldsymbol{G}_{h, S_{2}}\left(\Omega_{2} ; \rho_{2,0}\right):=\left\{\nabla \Phi_{2} \in \boldsymbol{L}_{2}\left(\Omega_{2} ; \rho_{2,0}\right): \operatorname{div}\left(\rho_{2,0} \nabla \Phi_{2}\right)=0 \text { в } \Omega_{2}, \rho_{2,0} \frac{\partial \Phi_{2}}{\partial n_{2}}=0 \text { на } S_{2}\right\} .
\end{aligned}
$$

Из (1.1.6), (1.1.7), (1.1.9) следует, что поле скоростей $\boldsymbol{u}_{2}(t, x), x \in \Omega_{2}$, для газа следует считать $($ при любом $t \geqslant 0)$ элементом подпространства $\boldsymbol{J}_{0}\left(\Omega_{2} ; \rho_{2,0}\right) \oplus \boldsymbol{G}_{h, S_{2}}\left(\Omega_{2} ; \rho_{2,0}\right)$.

1.2.3. Для поля давлений $p_{2}(t, x)$ в газе введем, опираясь на (1.1.20), гильбертово пространство

$$
L_{2}\left(\Omega_{2} ; \rho_{2,0}^{-1}\right):=\left\{p_{2}:\left\|p_{2}\right\|_{L_{2}\left(\Omega_{2} ; \rho_{2,0}^{-1}\right)}^{2}:=\int_{\Omega_{2}} \rho_{2,0}^{-1}(x)\left|p_{2}\right|^{2} d \Omega_{2}<\infty\right\}
$$

с нормой, эквивалентной норме $L_{2}\left(\Omega_{2}\right)$.

1.2.4. Далее, для конечности потенциальной энергии системы, отвечающей отклонению $\zeta$ движущейся границы раздела между жидкостью и газом, введем уже встретившееся выше (см. (1.2.6)) подпространство

$$
L_{2, \Gamma}:=\left\{\zeta \in L_{2}(\Gamma):\|\zeta\|_{L_{2, \Gamma}}^{2}:=\int_{\Gamma}|\zeta|^{2} d \Gamma<\infty, \int_{\Gamma} \zeta d \Gamma=0\right\}
$$

пространства $L_{2}(\Gamma)$.

1.2.5. Наконец, для конечности скорости диссипации энергии в вязкоупругой жидкости (снова см. (1.1.20)) введем пространство вектор-функций $\boldsymbol{H}^{1}\left(\Omega_{1}\right)$ и его подпространство

$$
\boldsymbol{J}_{0, S_{1}}^{1}\left(\Omega_{1}\right):=\left\{\boldsymbol{u}_{1} \in \boldsymbol{H}^{1}\left(\Omega_{1}\right): E_{1}\left(\boldsymbol{u}_{1} ; \boldsymbol{u}_{1}\right)<\infty, \boldsymbol{u}_{1}=\mathbf{0} \text { на } S_{1}, \operatorname{div} \boldsymbol{u}_{1}=0 \text { в } \Omega_{1}\right\} .
$$

Отметим, что норма в $\boldsymbol{J}_{0, S_{1}}^{1}\left(\Omega_{1}\right)$ эквивалентна стандартной норме пространства $\boldsymbol{H}^{1}\left(\Omega_{1}\right)$ при условии прилипания $\boldsymbol{u}_{1}=\mathbf{0}$ на $S_{1}$, так как в этом случае имеет место неравенство Корна (см. [13])

$$
\left\|\boldsymbol{u}_{1}\right\|_{\boldsymbol{J}_{0, S_{1}}^{1}\left(\Omega_{1}\right)}^{2}:=E_{1}\left(\boldsymbol{u}_{1}, \boldsymbol{u}_{1}\right) \geqslant c\left\|\boldsymbol{u}_{1}\right\|_{\boldsymbol{H}^{1}\left(\Omega_{1}\right)}^{2}:=c \sum_{k=1}^{3}\left\|u_{1, k}\right\|_{H^{1}\left(\Omega_{1}\right)}^{2}, \quad c>0 .
$$

Таким образом, далее в исследуемой проблеме искомые векторные и скалярные поля будем считать функциями переменной $t$ со значениями в соответствующих введенных выше пространствах и подпространствах. 


\section{3. Применение метода ортогонального проектирования для уравнений движения} газа. Введем согласно разложениям (1.2.7)-(1.2.9), ортопроекторы

$$
P_{0,2}: \boldsymbol{L}_{2}\left(\Omega_{2} ; \rho_{2,0}\right) \rightarrow \boldsymbol{J}_{0}\left(\Omega_{2} ; \rho_{2,0}\right), \quad P_{G, 2}: \boldsymbol{L}_{2}\left(\Omega_{2} ; \rho_{2,0}\right) \rightarrow \boldsymbol{G}\left(\Omega_{2} ; \rho_{2,0}\right)
$$

и перепишем уравнения движения газа (1.1.6), (1.1.7) с учетом (1.1.14) в виде

$$
\frac{\partial u_{2}}{\partial t}+\nabla\left(\rho_{2,0}^{-1} p_{2}\right)=\boldsymbol{f}_{2}, \quad a^{-2} \frac{\partial p_{2}}{\partial t}+\operatorname{div}\left(\rho_{2,0} \boldsymbol{u}_{2}\right)=0 .
$$

Будем теперь считать, что поле скоростей в газе имеет вид

$$
\boldsymbol{u}_{2}=\boldsymbol{w}_{2}+\nabla \Phi_{2}, \quad \boldsymbol{w}_{2} \in \boldsymbol{J}_{0}\left(\Omega_{2} ; \rho_{2,0}\right), \quad \nabla \Phi_{2} \in \boldsymbol{G}\left(\Omega_{2} ; \rho_{2,0}\right)
$$

(см. п. 1.2.2). Применяя ортопроекторы $P_{0,2}$ и $P_{G, 2}$ к левой и правой частям первого уравнения (1.3.1), получим два соотношения

$$
\frac{\partial \boldsymbol{w}_{2}}{\partial t}=P_{0,2} \boldsymbol{f}_{2}, \quad \frac{\partial}{\partial t} \nabla \Phi_{2}+\nabla\left(\rho_{2,0}^{-1} p_{2}\right)=P_{G, 2} \boldsymbol{f}_{2}=: \nabla F_{2} .
$$

Далее, в силу (1.2.8) второе уравнение (1.3.1) приобретает вид

$$
a^{-2} \frac{\partial p_{2}}{\partial t}+\operatorname{div}\left(\rho_{2,0} \nabla \Phi_{2}\right)=0
$$

Заметим теперь, что первое уравнение (1.3.2) тривиально разрешимо, если задано начальное условие $\boldsymbol{w}_{2}(0, x)=P_{0,2} \boldsymbol{u}_{2}^{0}(x)$. Поэтому в дальнейшем будем рассматривать связи для $p_{2}$ и $\Phi_{2}$, исходя из второго соотношения (1.3.2), уравнения (1.3.3) и с учетом краевых и начальных условий. В частности, из (1.1.9), определения (1.2.8) подпространства $\boldsymbol{J}_{0}\left(\Omega_{2} ; \rho_{2,0}\right)$ и из кинематического условия (1.1.10) имеем

$$
\frac{\partial \Phi_{2}}{\partial n}=-\frac{\partial \Phi_{2}}{\partial e_{3}}=-\boldsymbol{u}_{1} \cdot \boldsymbol{e}_{3}=-\frac{\partial \zeta}{\partial t} \quad \text { на } \Gamma, \quad \frac{\partial \Phi_{2}}{\partial n}=0 \quad \text { на } S_{2} .
$$

Отсюда получаем краевую задачу, описывающую связь функций $\Phi_{2}$ и $p_{2}$ :

$$
\begin{gathered}
-\Delta_{0} \Phi_{2}:=-\rho_{2,0}^{-1} \operatorname{div}\left(\rho_{2,0} \nabla \Phi_{2}\right)=a^{-2} \rho_{2,0}^{-1} \frac{\partial p_{2}}{\partial t}=: f \quad \text { в } \Omega_{2}, \\
\rho_{2,0} \frac{\partial \Phi_{2}}{\partial n}=0 \quad \text { на } S_{2}, \quad \rho_{2,0} \frac{\partial \Phi_{2}}{\partial n}=-\rho_{2,0}(0) \boldsymbol{u}_{1} \cdot \boldsymbol{e}_{3}=: \varphi \quad \text { на } \Gamma .
\end{gathered}
$$

Будем исследовать задачу (1.3.4), используя теорию обобщенных и слабых решений краевых задач в негладких (липшицевых) областях. Заметим, что однородная задача (1.3.4), т.е. отвечающая случаю $f=0, \varphi=0$, имеет нетривиальное решение $\Phi_{2}=c_{2}=$ const. Поэтому решение задачи (1.3.4) можно представить в виде

$$
\Phi_{2}=\Phi_{21}+\Phi_{22}+c_{2},
$$

где $\Phi_{21}$ и $\Phi_{22}$ - решения задач при однородном краевом условии и однородном уравнении соответственно:

$$
\begin{gathered}
-\Delta_{0} \Phi_{21}=f \quad \text { в } \Omega_{2}, \quad \rho_{2,0} \frac{\partial \Phi_{2}}{\partial n}=0 \quad \text { на } \partial \Omega_{2}, \quad \int_{\Omega_{2}} \rho_{2,0} \Phi_{21} d \Omega_{2}=0, \\
-\Delta_{0} \Phi_{22}=0 \quad \text { в } \Omega_{2}, \quad \rho_{2,0} \frac{\partial \Phi_{2}}{\partial n}=0 \quad \text { на } S_{2}, \quad \rho_{2,0} \frac{\partial \Phi_{2}}{\partial n}=\varphi \quad \text { на } \Gamma, \quad \int_{\Gamma} \Phi_{22} d \Gamma=0,
\end{gathered}
$$

a $c_{2}=$ const.

При изучении этих задач понадобится обобщенная формула Грина, аналогичная формуле (1.2.5), применительно к оператору $\Delta_{0}$ из (1.3.4). Отметим сначала, что необходимым условием разрешимости задачи (1.3.5) является условие

$$
\left(f, 1_{\Omega_{2}}\right)_{L_{2}\left(\Omega_{2} ; \rho_{2,0}\right)}:=\int_{\Omega_{2}} \rho_{2,0} f d \Omega_{2}=0 .
$$




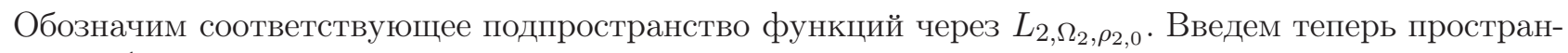
ство $H^{1}\left(\Omega_{2} ; \rho_{2,0}\right)$ с квадратом нормы

$$
\|\Phi\|_{H^{1}\left(\Omega_{2} ; \rho_{2,0}\right)}^{2}:=\int_{\Omega_{2}} \rho_{2,0}|\nabla \Phi|^{2} d \Omega_{2}+\left|\int_{\Omega_{2}} \rho_{2,0} \Phi d \Omega_{2}\right|^{2},
$$

эквивалентной норме $H^{1}\left(\Omega_{2}\right)$, и его подпространство $H_{\Omega_{2}}^{1}\left(\Omega_{2} ; \rho_{2,0}\right)$, состоящее из элементов, которые удовлетворяют условию (1.3.7), т.е.

$$
\int_{\Omega_{2}} \rho_{2,0} \Phi d \Omega_{2}=0
$$

Нетрудно видеть, что $\left(H_{\Omega_{2}}^{1}\left(\Omega_{2} ; \rho_{2,0}\right) ; L_{2, \Omega_{2}, \rho_{2,0}}\right)$ - гильбертова пара пространств, причем пространство $H_{\Omega_{2}}^{1}\left(\Omega_{2} ; \rho_{2,0}\right)$ компактно вложено в $L_{2, \Omega_{2}, \rho_{2,0}}$. Для элементов $\Psi_{2}$, $\Phi_{2}$ из $H_{\Omega_{2}}^{1}\left(\Omega_{2} ; \rho_{2,0}\right)$ имеет место обобщенная формула Грина

$$
\begin{aligned}
\left(\Psi_{2}, \Phi_{2}\right)_{H_{\Omega_{2}, \rho_{2}, 0}^{1}} & =\int_{\Omega_{2}} \rho_{2,0} \nabla \Psi_{2} \cdot \nabla \Phi_{2} d \Omega_{2}= \\
& =\left\langle\Psi_{2},-\Delta_{0} \Phi_{2}\right\rangle_{L_{2, \Omega_{2}, \rho_{2}, 0}}+\left\langle\gamma_{S_{2}} \Psi_{2}, \rho_{2,0} \frac{\partial \Phi_{2}}{\partial n}\right\rangle_{L_{2}\left(S_{2}\right)}+\left\langle\gamma_{\Gamma} \Phi_{2}, \rho_{2,0} \frac{\partial \Phi_{2}}{\partial n}\right\rangle_{L_{2}(\Gamma)},
\end{aligned}
$$

где $\gamma_{S_{2}}$ и $\gamma_{\Gamma}$-операторы следа на $S_{2}$ и $\Gamma$, а угловыми скобками обозначены значения соответствующих функционалов.

Уточним некоторые факты, связанные с формулой (1.3.9) (см. [8]). Она справедлива для тех элементов из $H_{\Omega_{2}}^{1}\left(\Omega_{2} ; \rho_{2,0}\right)$, которые обладают следующими свойствами: для этих элементов производные по нормали на $S_{2}$ и $\Gamma$ обладают тем свойством, что они продолжимы нулем с $S_{2}$ и $\Gamma$ соответственно на оставшуюся часть границы $\partial \Omega_{2}$ в классе функций из $H^{-1 / 2}\left(\partial \Omega_{2}\right)$. Соответствующую совокупность элементов из $H_{\Omega_{2}}^{1}\left(\Omega_{2} ; \rho_{2,0}\right)$ обозначим через $\check{H}_{\Omega_{2}}^{1}$, так что формула (1.3.9) справедлива для элементов из $\check{H}_{\Omega_{2}}^{1}$. При этом класс таких продолжимых нулем производных по нормали обозначается через $\tilde{H}^{-1 / 2}\left(S_{2}\right)$ и $\tilde{H}^{-1 / 2}(\Gamma)$ соответственно, причем эти подпространства являются сопряженными к $H^{1 / 2}\left(S_{2}\right)$ и $H^{1 / 2}(\Gamma)$ (см. $\left.[8,19,27]\right)$.

Опираясь на формулу Грина (1.3.9), определим слабое решение задачи (1.3.5) как элемент $\Phi_{21}$ из $H_{\Omega_{2}}^{1}\left(\Omega_{2} ; \rho_{2,0}\right)$, для которого выполнено тождество

$$
\left(\Psi_{2}, \Phi_{21}\right)_{H_{\Omega_{2}}^{1}\left(\Omega_{2}, \rho_{2,0}\right)}=\left\langle\Psi_{2}, f\right\rangle_{L_{2, \Omega_{2}, \rho_{2,0}}} \quad \forall \Psi_{2} \in H_{\Omega_{2}}^{1}\left(\Omega_{2} ; \rho_{2,0}\right) .
$$

Из теории пар гильбертовых пространств следует, что задача (1.3.5) имеет слабое решение тогда и только тогда, когда

$$
f \in\left(H_{\Omega_{2}}^{1}\left(\Omega_{2} ; \rho_{2,0}\right)\right)^{*} \supset L_{2, \Omega_{2}, \rho_{2,0}} .
$$

При $f \in L_{2, \Omega_{2}, \rho_{2,0}}$ эта задача имеет обобщенное решение, определяемое тождеством

$$
\left(\Psi_{2}, \Phi_{21}\right)_{H_{\Omega_{2}}^{1}\left(\Omega_{2} ; \rho_{2,0}\right)}=\left(\Psi_{2}, f\right)_{L_{2, \Omega_{2}, \rho_{2,0}}} \quad \forall \Psi_{2} \in H_{\Omega_{2}}^{1}\left(\Omega_{2} ; \rho_{2,0}\right) .
$$

Оба решения задач $(1.3 .10),(1.3 .11)$ выражаются одной формулой:

$$
\Phi_{21}=A_{2}^{-1} f
$$

где $A_{2}$-оператор гильбертовой пары $\left(H_{\Omega_{2}}^{1}\left(\Omega_{2} ; \rho_{2,0}\right) ; L_{2, \Omega_{2}, \rho_{2,0}}\right) ;$ при этом $\Phi_{21} \in \mathcal{D}\left(A_{2}^{1 / 2}\right)=$ $H_{\Omega_{2}}^{1}\left(\Omega_{2} ; \rho_{2,0}\right)$ для слабого решения и $\Phi_{21} \in \mathcal{D}\left(A_{2}\right) \subset \mathcal{D}\left(A_{2}^{1 / 2}\right)$ для обобщенного решения.

Таким образом, при $f \in L_{2, \Omega_{2}, \rho_{2,0}}$ задача (1.3.5) равносильна соотношению

где

$$
A_{2} \Phi_{21}=f=a^{-2} \rho_{2,0}^{-1} \frac{\partial p_{2}}{\partial t}
$$

$$
0 \ll A_{2}=A_{2}^{*}: \mathcal{D}\left(A_{2}\right) \rightarrow L_{2, \Omega_{2}, \rho_{2,0}}, \quad A_{2}^{-1} \in \mathfrak{S}_{\infty}\left(L_{2, \Omega_{2}, \rho_{2,0}}\right) .
$$


Перейдем теперь к рассмотрению задачи (1.3.6). Опираясь на формулу Грина (1.3.9), заметим сначала, что необходимым условием разрешимости этой задачи является условие

$$
\left\langle 1_{\Gamma}, \varphi\right\rangle_{L_{2}(\Gamma)}=0 .
$$

Если $\varphi \in L_{2}(\Gamma)$, то получаем условие

$$
\int_{\Gamma} \varphi d \Gamma=0
$$

т.е. условие ортогональности в $L_{2}(\Gamma)$ заданной функции $\varphi$ к единичной функции $1_{\Gamma}$, определенной на $Г$. В связи с этим введем в $H^{1}\left(\Omega_{2} ; \rho_{2,0}\right)$ норму

$$
\|\Phi\|_{H^{1}\left(\Omega_{2} ; \rho_{2,0}\right)}^{2}:=\int_{\Omega_{2}} \rho_{2,0}|\nabla \Phi|^{2} d \Omega_{2}+\left|\int_{\Gamma} \Phi d \Gamma\right|^{2},
$$

эквивалентную стандартной норме пространства $H^{1}\left(\Omega_{2}\right)$, и подпространство $H_{\Gamma}^{1}\left(\Omega_{2}, \rho_{2,0}\right)$ функций, для которых

$$
\int_{\Gamma} \Phi d \Gamma=0
$$

Снова опираясь на формулу Грина (1.3.9), определим слабое решение задачи (1.3.6) как элемент из $\Phi_{22}$ из $H_{\Gamma}^{1}\left(\Omega_{2} ; \rho_{2,0}\right)$, для которого выполнено тождество

$$
\left(\Psi_{2}, \Phi_{22}\right)_{H_{\Omega_{2}}^{1}\left(\Omega_{2}, \rho_{2,0}\right)}=\left\langle\gamma_{2} \Psi_{2}, \varphi\right\rangle_{L_{2}(\Gamma)}, \quad \forall \Psi_{2} \in H_{\Gamma}^{1}\left(\Omega_{2} ; \rho_{2,0}\right),
$$

где $\gamma_{2} \Psi_{2}:=\left.\Psi\right|_{\Gamma}$. Из (1.3.6) следует, что для слабого решения функция $\varphi$ должна быть продолжима нулем в классе $H^{-1 / 2}\left(\partial \Omega_{2}\right)$, т.е. необходимым и достаточным условия разрешимости задачи (1.3.6) является условие

$$
\varphi \in \tilde{H}_{\Gamma}^{-1 / 2}=\left(H_{\Gamma}^{1 / 2}\right)^{*}, \quad H_{\Gamma}^{1 / 2}:=H^{1 / 2}(\Gamma) \cap L_{2, \Gamma} .
$$

Тогда эта задача имеет единственное слабое решение

$$
\Phi_{22}=: V \varphi=V\left(-\rho_{2,0}(0) \boldsymbol{u}_{1} \cdot \boldsymbol{e}_{3}\right)=V\left(-\rho_{2,0}(0) \frac{\partial \zeta}{\partial t}\right), \quad V \in \mathcal{L}\left(\tilde{H}_{\Gamma}^{-1 / 2} ; H_{\Gamma, h}^{1}\left(\Omega_{2} ; \rho_{2,0}\right)\right),
$$

где

$$
\begin{gathered}
H_{\Gamma, h}^{1}\left(\Omega_{2} ; \rho_{2,0}\right)=\left\{\Phi_{22} \in H_{\Gamma}^{1}\left(\Omega_{2} ; \rho_{2,0}\right):-\Delta_{0} \Phi_{22}=0 \text { в } \Omega_{2},\right. \\
\left.\rho_{2,0} \frac{\partial \Phi_{22}}{\partial n}=0 \text { на } S_{2}, \int_{\Gamma} \rho_{2,0}(0) \Phi_{22} d \Gamma=0\right\} \subset H_{\Gamma}^{1}\left(\Omega_{2} ; \rho_{2,0}\right) .
\end{gathered}
$$

Заметим теперь, что для области $\Omega_{2}$ с липшицевой границей $\partial \Omega_{2}$, разбитой на липшицевы куски $S_{2}$ и $\Gamma$, имеет место теорема Гальярдо (см. [21]), согласно которой оператор следа $\gamma_{2}$ обладает свойством

$$
\gamma_{2} \in \mathcal{L}\left(H_{\Gamma}^{1}\left(\Omega_{2} ; \rho_{2,0}\right) ; H_{\Gamma}^{1 / 2}\right) .
$$

Отсюда получаем следующее утверждение.

Лемма 1.1. Oператоры

$$
V: \tilde{H}_{\Gamma}^{-1 / 2} \rightarrow H_{\Gamma, h}^{1}\left(\Omega_{2} ; \rho_{2,0}\right) \subset H_{\Gamma}^{1}\left(\Omega_{2} ; \rho_{2,0}\right), \quad \gamma_{2}: H_{\Gamma}^{1}\left(\Omega_{2} ; \rho_{2,0}\right) \rightarrow \tilde{H}_{\Gamma}^{-1 / 2}
$$

являются взаимно сопряюенными (ограниченными) операторами: $V=\gamma_{2}^{*}$. 
Доказательство следует из тождества (1.3.14) и определения оператора $V$ :

$$
\left(\Psi_{2}, V \varphi\right)_{H_{\Gamma}^{1}\left(\Omega_{2} ; \rho_{2,0}\right)}=\left\langle\gamma_{2} \Psi_{2}, \varphi\right\rangle_{L_{2, \Gamma}} .
$$

Итогом рассмотрения задачи (1.3.4) и вспомогательных задач (1.3.5), (1.3.6) является такой вывод: задача (1.3.4) имеет решение в пространстве $H^{1}\left(\Omega_{2} ; \rho_{2,0}\right)$, которое выражается формулой

$$
\left.\Phi_{2}\right|_{\Omega_{2}}=\Phi_{21}+\Phi_{22}+c_{2}=A_{2}^{-1}\left(a^{-2} \rho_{2,0}^{-1} \frac{\partial p_{2}}{\partial t}\right)+V\left(-\rho_{2,0}(0) \frac{\partial \zeta}{\partial t}\right)+c_{2},
$$

где $c_{2}$ - произвольная функция $t$.

1.4. Преобразования операторными методами уравнений движения жидкости. Преобразуем теперь уравнения движения жидкости (1.1.5) в области $\Omega_{1}$ с учетом граничных условий (1.1.8), (1.1.11). Опираясь на ортогональные разложения (1.2.1)-(1.2.4), будем считать при каждом $t$ поле $\boldsymbol{u}_{1}(t, x)$ элементом подпространства $\boldsymbol{J}_{0, S_{1}}^{1}\left(\Omega_{1}\right) \subset \boldsymbol{J}_{0, S_{1}}\left(\Omega_{1}\right)$, а $\nabla p_{1}$ - элементом подпространства $\boldsymbol{G}\left(\Omega_{1}\right)=\boldsymbol{G}_{0, \Gamma}\left(\Omega_{1}\right) \oplus \boldsymbol{G}_{h, S_{1}}\left(\Omega_{1}\right)$. Введем далее ортопроектор

$$
P_{0, S_{1}}: \boldsymbol{L}_{2}\left(\Omega_{1}\right) \rightarrow \boldsymbol{J}_{0, S_{1}}\left(\Omega_{1}\right)
$$

и подействуем им на обе части уравнения (1.1.5). Будем иметь соотношения

$$
\begin{gathered}
\rho_{1} \frac{\partial \boldsymbol{u}_{1}}{\partial t}-\mu_{1} P_{0, S_{1}} \Delta \boldsymbol{v}_{1}+\nabla \tilde{p}_{1}=\rho_{1} P_{0, S_{1}} \boldsymbol{f}_{1}, \quad \nabla \tilde{p}_{1}:=P_{0, S_{1}} \nabla p_{1}, \\
\Delta \tilde{p}_{1}=0 \quad \text { в } \Omega_{1}, \quad \frac{\partial \tilde{p}_{1}}{\partial n}=0 \quad \text { на } S_{1}, \quad \int_{\Gamma} \tilde{p}_{1} d \Gamma=0, \\
\boldsymbol{v}_{1}=\mathbf{0} \quad \text { на } S_{1}, \quad \mu_{1} \tau_{j 3}\left(\boldsymbol{v}_{1}\right)=0, \quad j=1,2, \quad \text { на } \Gamma, \\
{\left[-\tilde{p}_{1} \mu_{1} \tau_{33}\left(\boldsymbol{v}_{1}\right)\right]-\left[-p_{2}\right]=-g\left(\rho_{1}-\rho_{2,0}(0)\right) \zeta \quad \text { на } \Gamma .}
\end{gathered}
$$

Здесь уже учтено, что в силу (1.2.4) имеем $p_{1}=\tilde{p}_{1}$ на $\Gamma$.

Представим теперь поле $\nabla \tilde{p}_{1}$ в виде

$$
\nabla \tilde{p}_{1}=\nabla p_{11}+\nabla p_{12}, \quad \nabla p_{1 l} \in \boldsymbol{G}_{h, S_{1}}\left(\Omega_{1}\right), \quad l=1,2,
$$

и рассмотрим две вспомогательные краевые задачи, сумма решений которых дает решение задачи (1.4.1)-(1.4.3).

Первая вспомогательная задача (ее называют задачей С. Г. Крейна, см. [13, 14]):

$$
\begin{array}{r}
-\mu_{1} P_{0, S_{1}} \Delta \boldsymbol{v}_{1}+\nabla p_{11}=\boldsymbol{F}_{1}:=-\nabla p_{12}-\rho_{1} \frac{\partial \boldsymbol{u}_{1}}{\partial t}+\rho_{1} P_{0, S_{1}} \boldsymbol{f}_{1}, \\
\operatorname{div} \boldsymbol{v}_{1}=0 \quad \text { в } \Omega_{1}, \quad \boldsymbol{v}_{1}=\mathbf{0} \quad \text { на } S_{1}, \\
\mu_{1} \tau_{j 3}\left(\boldsymbol{v}_{1}\right)=0 \quad \text { на } \Gamma, \quad j=1,2 ;
\end{array}
$$

Вторая вспомогательная задача (задача Зарембы):

$$
\Delta p_{12}=0 \quad \text { в } \Omega_{1}, \quad \frac{\partial p_{12}}{\partial n}=0 \quad \text { на } S_{1}, \quad p_{12}=p_{2}+g\left(\rho_{1}-\rho_{2,0}(0)\right) \zeta=: \eta \quad \text { на } \Gamma .
$$

Обсудим сначала слабые решениях задачи (1.4.5). Заметим предварительно, что в силу условий

$$
\int_{\Gamma} p_{12} d \Gamma=0, \quad \int_{\Gamma} \zeta d \Gamma=0
$$

возникает требование нормировки для давления в газе:

$$
\int_{\Gamma} p_{2} d \Gamma=0 .
$$

Тогда задача Зарембы (1.4.5) имеет при $\eta \in H_{\Gamma}^{1 / 2}$ единственное решение $p_{12}$, для которого

$$
\nabla p_{12}=: Q \eta=Q\left(\left.p_{2}\right|_{\Gamma}+g\left(\rho_{1}-\rho_{2,0}(0)\right) \zeta\right), \quad Q \in \mathcal{L}\left(H_{\Gamma}^{1 / 2} ; \boldsymbol{G}_{h, S_{1}}\left(\Omega_{1}\right)\right) .
$$


Чтобы получить окончательное выражение для $\nabla p_{12}$, найдем значение $\left.p_{2}\right|_{\Gamma}$ с учетом нормировки (1.4.6). С этой целью воспользуемся следствием из второй формулы (1.3.2), которое называют интегралом Коши-Лагранжа:

$$
\frac{\partial \Phi_{2}}{\partial t}+\rho_{2,0}^{-1} p_{2}=F_{2}+c(t) \quad \text { в } \Omega_{2},
$$

где $c(t)$ - произвольная функция $t$. Отсюда получаем, что

$$
\left.p_{2}\right|_{\Gamma}=\rho_{2,0}(0) \gamma_{2}\left(-\frac{\partial \Phi_{2}}{\partial t}+F_{2}\right)+\rho_{2,0}(0) c(t),
$$

откуда с учетом (1.3.18) приходим к формуле

$$
\left.p_{2}\right|_{\Gamma}=\rho_{2,0}(0) \gamma_{2}\left\{-\frac{\partial}{\partial t} \Phi_{21}-\rho_{2,0}(0) V \gamma_{n, 1} \boldsymbol{u}_{1}\right\}+\rho_{2,0}(0)\left(\gamma_{2} F_{2}+c(t)\right) .
$$

Наконец, учитывая интегральное условие (1.4.6), окончательно имеем

$$
\left.p_{2}\right|_{\Gamma}=-\rho_{2,0}(0) \frac{\partial}{\partial t}\left\{P_{\Gamma} \gamma_{2} \nabla^{-1}\left(\nabla \Phi_{21}\right)-\rho_{2,0}(0) \gamma_{2} V \gamma_{n, 1} \boldsymbol{u}_{1}\right\}+\rho_{2,0}(0) P_{\Gamma} \gamma_{2} F_{2},
$$

где $P_{\Gamma}: L_{2}(\Gamma) \rightarrow L_{2, \Gamma}$ - ортопроектор.

Рассмотрим теперь первую вспомогательную задачу (1.4.4), считая $\boldsymbol{F}_{1}$ заданным элементом. Здесь понадобится обобщенная формула Грина для соленоидальных полей и векторного оператора Лапласа. Для гладких полей она имеет вид (1.1.15), а для полей из $\boldsymbol{J}_{0, S_{1}}^{1}\left(\Omega_{1}\right)$ эта формула приобретает вид (см. $[8,10])$

$$
\begin{aligned}
\mu_{1} E_{1}\left(\boldsymbol{u}_{1}, \boldsymbol{v}_{1}\right)=\left\langle\boldsymbol{u}_{1},-\mu_{1} P_{0, S_{1}} \Delta \boldsymbol{v}_{1}\right. & \left.+\nabla p_{1}\right\rangle_{\boldsymbol{L}_{2}\left(\Omega_{1}\right)}+ \\
& \left.+\sum_{j, k=1}^{3}\left\langle u_{1 j} \cos \widehat{\left(\boldsymbol{n}, \boldsymbol{e}_{3}\right.}\right),\left[\tau_{j k}\left(\boldsymbol{v}_{1}\right)-p_{1} \delta_{j k}\right]\right\rangle_{L_{2}(\Gamma)} .
\end{aligned}
$$

Используя формулу (1.4.9), определим слабое решение задачи (1.4.4) как элемент $\boldsymbol{v}_{1} \in \boldsymbol{J}_{0, S_{1}}^{1}\left(\Omega_{1}\right)$, для которого выполнено тождество

$$
\mu_{1} E_{1}\left(\boldsymbol{\eta}_{1}, \boldsymbol{v}_{1}\right)=\left\langle\boldsymbol{\eta}_{1}, \boldsymbol{F}_{1}\right\rangle_{\boldsymbol{L}_{2}\left(\Omega_{1}\right)} \forall \boldsymbol{\eta}_{1} \in \boldsymbol{J}_{0, S_{1}}^{1}\left(\Omega_{1}\right) .
$$

Заметим теперь, что в силу неравенства Корна (1.2.12) пространство $J_{0, S_{1}}^{1}\left(\Omega_{1}\right)$ компактно вложено в $J_{0, S_{1}}\left(\Omega_{1}\right)$. Поэтому эти пространства образуют гильбертову пару $\left(J_{0, S_{1}}^{1}\left(\Omega_{1}\right) ; J_{0, S_{1}}\left(\Omega_{1}\right)\right)$, причем оператор этой гильбертовой пары

$$
A_{1}: \mathcal{D}\left(A_{1}\right) \subset \boldsymbol{J}_{0, S_{1}}\left(\Omega_{1}\right) \rightarrow \boldsymbol{J}_{0, S_{1}}\left(\Omega_{1}\right)
$$

неограничен, положительно определен и имеет компактный обратный оператор $A_{1}^{-1}$. При этом

$$
E_{1}\left(\boldsymbol{\eta}_{1}, \boldsymbol{v}_{1}\right)=\left(\boldsymbol{\eta}_{1}, \boldsymbol{v}_{1}\right)_{\boldsymbol{J}_{0, S_{1}}^{1}\left(\Omega_{1}\right)}=\left(A^{1 / 2} \boldsymbol{\eta}_{1}, A_{1}^{1 / 2} \boldsymbol{v}_{1}\right)_{\boldsymbol{J}_{0, S_{1}}\left(\Omega_{1}\right)}=\left\langle\boldsymbol{\eta}_{1}, A_{1} \boldsymbol{v}_{1}\right\rangle_{\boldsymbol{J}_{0, S_{1}}\left(\Omega_{1}\right)},
$$

для всех $\boldsymbol{\eta}_{1}, \boldsymbol{v}_{1} \in \boldsymbol{J}_{0, S_{1}}^{1}\left(\Omega_{1}\right)$.

Эти рассуждения показывают, что задача (1.4.4) имеет слабое решение $\boldsymbol{v}_{1} \in \boldsymbol{J}_{0, S_{1}}^{1}\left(\Omega_{1}\right)$ тогда и только тогда, когда выполнено условие

$$
\boldsymbol{F}_{1} \in\left(\boldsymbol{J}_{0, S_{1}}^{1}\left(\Omega_{1}\right)\right)^{*}
$$

и это решение выражается формулой

$$
\boldsymbol{v}_{1}=\mu_{1}^{-1} A_{1}^{-1} \boldsymbol{F}_{1} .
$$

Если $\boldsymbol{F}_{1} \in \boldsymbol{J}_{0, S_{1}}\left(\Omega_{1}\right) \subset\left(\boldsymbol{J}_{0, S_{1}}^{1}\left(\Omega_{1}\right)\right)^{*}$, то формула (1.4.12) дает обобщенное решение задачи (1.4.4), и при этом $\boldsymbol{v}_{1} \in \mathcal{D}\left(A_{1}\right) \subset \mathcal{D}\left(A_{1}^{1 / 2}\right)=\boldsymbol{J}_{0, S_{1}}^{1}\left(\Omega_{1}\right)$.

Формула (1.4.12) позволяет с учетом определения $\boldsymbol{F}_{1}$ (см. (1.4.4)) выписать дифференциальнооператорную связь между искомыми функциями в исследуемой проблеме и данными задачи. Как уже упоминалось выше, все эти функции считаем функциями переменной $t$ со значениями 
в соответствующем гильбертовом пространстве; в соответствии с этим далее производные $\partial / \partial t$ заменим на $d / d t$.

Подставляя выражение для $\boldsymbol{F}_{1}$ в соотношение $\mu_{1} A_{1} \boldsymbol{v}_{1}=\boldsymbol{F}_{1}$, вытекающее из (1.4.11), и используя формулы (1.4.7) и (1.4.8) для $\nabla p_{12}$ и $\left.p_{2}\right|_{\Gamma}$, приходим к соотношению

$$
\begin{aligned}
\frac{d}{d t}\left\{\left[\rho_{1} \boldsymbol{u}_{1}+\left(\rho_{2,0}(0)\right)^{2}\left(Q \gamma_{2}\right)\right.\right. & \left.\left.\left(V \gamma_{n, 1}\right) \boldsymbol{u}_{1}\right]-\rho_{2,0}(0)\left(Q P_{\Gamma} \gamma_{2} \nabla^{-1}\right)\left(\nabla \Phi_{21}\right)\right\}+ \\
+\mu_{1} A_{1} \boldsymbol{v}_{1}+g\left(\rho_{1}-\rho_{2,0}(0)\right) Q \zeta & =\rho_{1} P_{0, S_{1}} \boldsymbol{f}_{1}-\rho_{2,0}(0) Q P_{\Gamma} \gamma_{2} F_{2},
\end{aligned}
$$

где

$$
\gamma_{n, 1} \boldsymbol{u}_{1}:=\left(\boldsymbol{u}_{1} \cdot \boldsymbol{n}\right)_{\Gamma}, \quad \boldsymbol{v}_{1}=\boldsymbol{u}_{1}(t, x)+\sum_{k=1}^{m} \alpha_{k} \int_{0}^{t} e^{-\beta_{k}(t-s)} \boldsymbol{u}_{1}(s, x) d s:=I_{01}(t) \boldsymbol{u}_{1} .
$$

Выпишем теперь дополнительно к (1.4.13) другие соотношения, связанные с изучаемой задачей. Перепишем кинематические условия (1.1.10) в эквивалентной форме:

$$
g\left(\rho_{1}-\rho_{2,0}(0)\right) \frac{d \zeta}{d t}-g\left(\rho_{1}-\rho_{2,0}(0)\right) \gamma_{n, 1} \boldsymbol{u}_{1}=0 ;
$$

приведем также выведенные ранее соответствующие связи (1.3.3), (1.3.12) для газа и учтем формулу (1.3.15). Будем иметь соотношения

$$
\begin{gathered}
\frac{d}{d t}\left(\nabla \Phi_{21}-\rho_{2,0}(0) \nabla\left(V \gamma_{n, 1} \boldsymbol{u}_{1}\right)\right)+\nabla\left(\rho_{2,0}(0)^{-1} p_{2}\right)=P_{G, 2} \boldsymbol{f}_{2}=: \nabla F_{2}, \\
a^{-2} \frac{d p_{2}}{d t}+\operatorname{div}\left(\rho_{2,0} \nabla \Phi_{21}\right)=0 .
\end{gathered}
$$

Итогом проведенных рассуждений является следующее утверждение. Исходная начально-краевая задача о малых движениях системы, состоящей из вязкоупругой жидкости и баротропного газа, заполняющих произвольный неподвижный сосуд, приводится, кроме тривиальной связи (1.3.2) (первое уравнение), к задаче Коши для системы дифференциально-операторных уравнений (1.4.13)-(1.4.16) с соответствующими начальными условиями. Искомыми функциями являются: поле скоростей $\boldsymbol{u}_{1}(t, x)$ со значениями в $\boldsymbol{J}_{0, S_{1}}\left(\Omega_{1}\right)$, вертикальное отклонение $\zeta\left(t, x_{1}, x_{2}\right)$, $\left(x_{1}, x_{2}\right) \in \Gamma$, границы раздела со значениями в $L_{2, \Gamma}$, потенциальная компонента $\nabla \Phi_{21}(t, x)$, описывающая одно из полей скоростей газа, со значениями в $\boldsymbol{G}\left(\Omega_{1} ; \rho_{2,0}\right)$, а также поле давлений в газе $p_{2}(t, x)$ со значениями в $L_{2}\left(\Omega_{1} ; \rho_{2,0}^{-1}\right)$.

1.5. Формулировка задачи в векторно-матричной форме. Свойства операторных коэффициентов задачи. Перепишем задачу (1.4.13)-(1.4.16) в векторно-матричном виде, введя в качестве искомого объекта вектор-столбец

$$
z(t):=\left(\boldsymbol{u}_{1} ; \zeta ; \nabla \Phi_{21} ; p_{2}\right)^{\tau}
$$

со значениями в пространстве

$$
\mathcal{H}:=\boldsymbol{J}_{0, S_{1}}\left(\Omega_{1}\right) \oplus L_{2, \Gamma} \oplus \boldsymbol{G}\left(\Omega_{2} ; \rho_{2,0}\right) \oplus L_{2}\left(\Omega_{2} ; \rho_{2,0}^{-1}\right) .
$$

Введем операторные матрицы

$$
C=\left(\begin{array}{cccc}
\rho_{1} I_{1}+\left(\rho_{2,0}\right)^{2}\left(Q \gamma_{2}\right)\left(V \gamma_{n, 1}\right) & 0 & -\rho_{2,0}(0) Q P_{\Gamma} \gamma_{2} \nabla^{-1} & 0 \\
0 & g\left(\rho_{1}-\rho_{2,0}(0)\right) I_{2} & 0 & 0 \\
-\rho_{2,0}(0) \nabla V \gamma_{n, 1} & 0 & I_{3} & 0 \\
0 & 0 & 0 & a^{-2} I_{4}
\end{array}\right),
$$

где $I_{k}$ - единичные операторы в пространствах (1.5.1),

$$
\begin{aligned}
& B=\left(\begin{array}{cccc}
\mu_{1} A_{1} I_{01}(t) & g\left(\rho_{1}-\rho_{2,0}(0)\right) Q & 0 & 0 \\
-g\left(\rho_{1}-\rho_{2,0}(0)\right) \gamma_{n, 1} & 0 & 0 & 0 \\
0 & 0 & 0 & B_{34} \\
0 & 0 & B_{43} & 0
\end{array}\right), \\
& B_{34} p_{2}:=\nabla\left(\rho_{2,0}^{-1} p_{2}\right), \quad B_{43}\left(\nabla \Phi_{21}\right):=\operatorname{div}\left(\rho_{2,0} \nabla \Phi_{21}\right),
\end{aligned}
$$


а также вектор-столбец заданных функций:

$$
f:=\left(\rho_{1} P_{0, S_{1}} \boldsymbol{f}_{1}-\rho_{2,0}(0) Q P_{\Gamma} \gamma_{2} F_{2} ; 0 ; \nabla F_{2} ; 0\right)^{\tau} .
$$

Тогда систему дифференциальных уравнений (1.4.13)-(1.4.16) вместе с начальными условиями можно коротко записать в виде задачи Коши для интегро-дифференциального уравнения первого порядка в пространстве $\mathcal{H}$ :

$$
C \frac{d z}{d t}+B z=f, \quad z(0)=z^{0} .
$$

Цель дальнейших рассмотрений - изучить свойства коэффициентов операторных матриц $C$ и $B$ и преобразовать задачу (1.5.3) таким образом, чтобы можно было доказать теорему существования ее решения на произвольном отрезке времени и на этой основе доказать теорему существования решения исходной начально-краевой задачи (1.1.5)-(1.1.13).

Напомним сначала, что согласно лемме 2.3 операторы

$$
V \in \mathcal{L}\left(\tilde{H}_{\Gamma}^{-1 / 2} ; H_{\Gamma, h}^{1}\left(\Omega_{2} ; \rho_{2,0}\right)\right), \quad \text { и } \quad \gamma_{2} \in \mathcal{L}\left(H_{\Gamma}^{1}\left(\Omega_{2} ; \rho_{2,0}\right) ; H_{\Gamma}^{1 / 2}\right)
$$

взаимно сопряжены. Докажем теперь аналогичное свойство для операторов $Q$ и $\gamma_{n, 1}$ (см. (1.4.7), (1.4.13), (1.4.14)).

Лемма 1.2. Операторь

$$
Q \in \mathcal{L}\left(H_{\Gamma}^{1 / 2} ; \boldsymbol{G}_{h, S_{1}}\left(\Omega_{1}\right)\right) \quad u \quad \gamma_{n, 1} \in \mathcal{L}\left(\boldsymbol{G}_{h, S_{1}}\left(\Omega_{1}\right) ; \tilde{H}_{\Gamma}^{-1 / 2}\right)
$$

взаимно сопряжены.

Доказательство. Пусть $\eta$ произвольный элемент из $H_{\Gamma}^{1 / 2}$, а $\boldsymbol{u}_{1}=\boldsymbol{w}_{1}+\nabla \Phi_{1}$ - гладкий элемент из $\boldsymbol{J}_{0, S_{1}}\left(\Omega_{1}\right)=\boldsymbol{J}_{0}\left(\Omega_{1}\right) \oplus \boldsymbol{G}_{h, S_{1}}\left(\Omega_{1}\right)$. Тогда, согласно (1.4.7), $Q \eta=\nabla p \in \boldsymbol{G}_{h, S_{1}}\left(\Omega_{1}\right)$. Опираясь на свойства элементов из $\boldsymbol{J}_{0}\left(\Omega_{1}\right)$, будем иметь

$$
\begin{aligned}
\left(Q \eta, \boldsymbol{u}_{1}\right)_{\boldsymbol{L}_{2}\left(\Omega_{1}\right)}=\int_{\Omega_{1}} \nabla p \cdot \overline{\boldsymbol{u}_{1}} t d \Omega_{1}=\int_{\Omega_{1}} \operatorname{div}\left(p \overline{\boldsymbol{u}_{1}}\right) d \Omega_{1}-\int_{\Omega_{1}} p \operatorname{div} \boldsymbol{u}_{1} d \Omega_{1}= \\
\quad=\int_{\Gamma} p\left(\boldsymbol{u}_{1} \cdot \boldsymbol{n}\right) d \Gamma=\left\langle\eta, \gamma_{n, 1} \boldsymbol{u}_{1}\right\rangle_{L_{2, \Gamma}} .
\end{aligned}
$$

Замыкая это тождество с гладких $\boldsymbol{u}_{1}$ на произвольные элементы из $\boldsymbol{J}_{0, S_{1}}\left(\Omega_{1}\right)$, приходим к выводу, что $\gamma_{n, 1} \in \mathcal{L}\left(\boldsymbol{G}_{h, S_{1}}\left(\Omega_{1}\right) ; \tilde{H}_{\Gamma}^{-1 / 2}\right)$ и $Q^{*}=\gamma_{n, 1}$.

Из лемм 1.1 и 1.2 вытекает следующее утверждение.

Лемма 1.3. Оператор $\left(Q \gamma_{2}\right)\left(V \gamma_{n, 1}\right)$ является ограниченным неотрицательным оператором, действующим в пространстве $\boldsymbol{J}_{0, S_{1}}\left(\Omega_{1}\right) \supset \boldsymbol{G}_{h, S_{1}}\left(\Omega_{1}\right)$.

Доказательство. В самом деле, на подпространстве $\boldsymbol{J}_{0}\left(\Omega_{1}\right)=\boldsymbol{J}_{0, S_{1}}\left(\Omega_{1}\right) \ominus \boldsymbol{G}_{h, S_{1}}\left(\Omega_{1}\right)$ этот оператор нулевой, а на $\boldsymbol{G}_{h, S_{1}}\left(\Omega_{1}\right)$ он положителен. Кроме того, в силу свойств $Q, \gamma_{2}, V$ и $\gamma_{n, 1}$ получаем, что

$$
Q \gamma_{2} V \gamma_{n, 1} \in \mathcal{L}\left(\boldsymbol{J}_{0, S_{1}}\left(\Omega_{1}\right), \boldsymbol{J}_{0, S_{1}}\left(\Omega_{1}\right)\right), \quad \mathcal{R}\left(Q \gamma_{2} V \gamma_{n, 1}\right) \subset \boldsymbol{G}_{h, S_{1}}\left(\Omega_{1}\right) \subset \boldsymbol{J}_{0, S_{1}}\left(\Omega_{1}\right)
$$

Лемма 1.4. Onераторы

$$
Q P_{\Gamma} \gamma_{2} \nabla^{-1}: \boldsymbol{G}\left(\Omega_{2} ; \rho_{2,0}\right) \rightarrow \boldsymbol{G}_{h, S_{1}}\left(\Omega_{1}\right) \quad \text { u } \quad \nabla V \gamma_{n, 1}: \boldsymbol{J}_{0, S_{1}}\left(\Omega_{1}\right) \rightarrow \boldsymbol{G}_{h, \Gamma}\left(\Omega_{2} ; \rho_{2,0}\right) \subset \boldsymbol{G}\left(\Omega_{2} ; \rho_{2,0}\right)
$$

взаимно сопря⿻сены и ограничены:

$$
Q P_{\Gamma} \gamma_{2} \nabla^{-1} \in \mathcal{L}\left(\boldsymbol{G}\left(\Omega_{2} ; \rho_{2,0}\right) ; \boldsymbol{G}_{h, S_{1}}\left(\Omega_{1}\right)\right), \quad \nabla V \gamma_{n, 1} \in \mathcal{L}\left(\boldsymbol{J}_{0, S_{1}}\left(\Omega_{1}\right) ; G_{h, \Gamma}\left(\Omega_{2} ; \rho_{2,0}\right)\right) .
$$


Доказательство. Проверим сначала, что эти операторы обладают свойствами (1.5.5). В самом деле, пусть $\nabla \Phi_{21}$ - элемент из $\boldsymbol{G}_{0}\left(\Omega_{2} ; \rho_{2,0}\right)$. Тогда $\left.\Phi_{21}\right|_{\Omega_{2}}=\nabla^{-1}\left(\nabla \Phi_{21}\right)$ - элемент из $H_{\Omega_{2}}^{1}\left(\Omega_{2} ; \rho_{2,0}\right)$, $\gamma_{2} \nabla^{-1}\left(\Delta \Phi_{21}\right) \in H^{1 / 2}(\Gamma)$ (теорема Гальярдо), $P_{\Gamma} \gamma_{2} \nabla^{-1}\left(\nabla \Phi_{21}\right) \in L_{2, \Gamma} \cap H^{1 / 2}(\Gamma)=H_{\Gamma}^{1 / 2}$, $Q P_{\Gamma} \gamma_{2} \nabla^{-1}\left(\nabla \Phi_{21}\right) \in \boldsymbol{G}_{h, S_{1}}\left(\Omega_{1}\right)$ (см. (1.4.7)), причем каждый из операторов является непрерывным из одного пространства в другое.

Аналогично можно проверить второе свойство (1.5.5). Действительно, если $\boldsymbol{u}_{1}$ - произвольный элемент из $\boldsymbol{J}_{0, S_{1}}\left(\Omega_{1}\right)$, то $\gamma_{n, 1} \boldsymbol{u}_{1} \in \tilde{H}_{\Gamma}^{-1 / 2}, V \gamma_{n, 1} \boldsymbol{u}_{1} \in H_{\Gamma, h}^{1}\left(\Omega_{2} ; \rho_{2,0}\right)$ (см. (1.3.15), (1.3.16)), $\nabla\left(V \gamma_{n, 1} \boldsymbol{u}_{1}\right) \in \boldsymbol{G}_{\Omega_{2}}\left(\Omega_{2} ; \rho_{2,0}\right)$ и является градиентом слабого решения задачи (1.3.5). Здесь снова все переходы осуществляются с помощью операторов, ограниченных из одного пространства в другое.

Докажем теперь взаимную сопряженность этих операторов. Пусть

$$
\boldsymbol{u}_{1}=\nabla \psi_{1} \in \boldsymbol{G}_{h, S_{1}}\left(\Omega_{1}\right) \subset \boldsymbol{J}_{0, S_{1}}\left(\Omega_{1}\right), \quad \nabla \Phi_{21} \in \boldsymbol{G}\left(\Omega_{2} ; \rho_{2,0}\right) .
$$

Тогда

$$
\int_{\Omega_{2}} \rho_{2,0} \nabla\left(V\left(-\rho_{2,0}(0)\right) \gamma_{n, 1} \boldsymbol{u}_{1}\right) \cdot \nabla \Phi_{21} d \Omega_{2}=\int_{\Omega_{2}} \rho_{2,0} \nabla \Phi_{22} \cdot \overline{\nabla \Phi_{21}} d \Omega_{2},
$$

где $\Phi_{22} \in H_{\Gamma, h}^{1}\left(\Omega_{2} ; \rho_{2,0}\right)$ - слабое решение задачи (1.3.6) при $\varphi=-\rho_{2,0}(0)\left(\boldsymbol{u}_{1} \cdot \boldsymbol{e}_{3}\right)_{\Gamma}=-\rho_{2,0}(0) \gamma_{n, 1} \boldsymbol{u}_{1}$ (см. (1.3.15)).

Далее, для другого оператора из (1.5.5) имеем для гладкого $\boldsymbol{u}_{1}=\nabla \psi_{1} \in \boldsymbol{G}_{h, S_{1}}\left(\Omega_{1}\right)$ :

$$
\begin{array}{r}
\int_{\Omega_{1}}\left[\left(-\rho_{2,0}(0)\right) Q P_{\Gamma} \gamma_{2} \nabla^{-1}\left(\nabla \Phi_{21}\right)\right] \cdot \overline{\nabla \psi_{1}} d \Omega_{1}=\int_{\Gamma}\left(P_{\Gamma} \gamma_{2} \Phi_{21}\right)\left(-\rho_{2,0}(0)\right) \gamma_{n, 1} \overline{\boldsymbol{u}_{1}} d \Gamma= \\
=\int_{\Gamma}\left(\gamma_{2} \Phi_{21}\right)\left(-\rho_{2,0}(0)\right) \gamma_{n, 1} \overline{\boldsymbol{u}_{1}} d \Gamma=\left(\Phi_{21}, V\left(-\rho_{2,0}(0)\right) \gamma_{n, 1} \overline{\boldsymbol{u}_{1}}\right)_{H_{\Gamma}^{1}\left(\Omega_{2} ; \rho_{2,0}\right)}= \\
=\int_{\Omega_{2}} \rho_{2,0} \nabla \Phi_{21} \cdot \overline{\nabla \Phi_{22}} d \Omega_{2} .
\end{array}
$$

Здесь при выводе были использованы следующие свойства: взаимная сопряженность $Q$ и $\gamma_{n, 1}$, свойства $P_{\Gamma}=P_{\Gamma}^{*}$ и $P_{\Gamma} \gamma_{n, 1} \boldsymbol{u}_{1}=\gamma_{n, 1} \boldsymbol{u}_{1}$, а также взаимная сопряженность $\gamma_{2}$ и $V$.

Переходя в (1.5.7) от гладких $\boldsymbol{u}_{1}=\nabla \psi_{1}$ к произвольным элементам из $\boldsymbol{G}_{h, S_{1}}\left(\Omega_{1}\right)$, убеждаемся, что тождество (1.5.7) справедливо и для произвольных $\nabla \psi_{1}$. Сравнивая тогда (1.5.6) и (1.5.7), получаем, что операторы (1.5.5) взаимно сопряжены.

Опираясь на доказанные свойства элементов операторной матрицы $C$ из (1.5.2), установим теперь общие свойства этой матрицы как оператора, действующего в пространстве $\mathcal{H}$ (см. (1.5.1)).

Лемма 1.5. Операторная матрица $C: \mathcal{H} \rightarrow \mathcal{H}$ является ограниченным положительно определенным оператором, ее квадратичная форма равна удвоенной полной энергии исследуемой гидромеханической системы и отвечает потенииальным движениям баротропного газа и произвольным движениям вязкоупругой жидкости.

Доказательство. Факт ограниченности операторной матрицы $C$ следует из лемм 2.3, 1.2-1.4 и ее вида (1.5.2). Вычислим квадратичную форму матрицы $C$, учитывая свойства операторных коэффициентов, формулы (1.5.6), (1.5.7), а также связь (1.3.15), т.е.

$$
\left.\Phi_{22}\right|_{\Omega_{2}}=V\left(-\rho_{2,0}(0) \gamma_{n, 1} \boldsymbol{u}_{1}\right),
$$

из которой следует соотношение

$$
\left(\rho_{2,0}(0)\right)^{2} \int_{\Omega_{1}}\left(Q \gamma_{2}\right)\left(V \gamma_{n, 1} \boldsymbol{u}_{1}\right) \cdot \overline{\boldsymbol{u}_{1}} d \Omega_{1}=\int_{\Omega_{2}} \rho_{2,0}\left|\nabla \Phi_{22}\right|^{2} d \Omega_{2} .
$$

Отсюда получаем формулу 


$$
\begin{aligned}
(C z, z)_{\mathcal{H}}=\rho_{1} \int_{\Omega_{1}}\left|\boldsymbol{u}_{1}\right|^{2} d \Omega_{1}+\int_{\Omega_{2}} \rho_{2,0} \mid \nabla \Phi_{21}+ & \left.\nabla \Phi_{22}\right|^{2} d \Omega_{2}+ \\
& +g\left(\rho_{1}-\rho_{2,0}(0)\right) \int_{\Gamma}|\zeta|^{2} d \Gamma+a^{-2} \int_{\Omega_{2}}\left|p_{2}\right|^{2} d \Omega_{2},
\end{aligned}
$$

в которой правая часть есть удвоенная полная энергия гидросистемы, отвечающая потенциальным движениям газа с полем скорости $\nabla \Phi_{2}=\nabla \Phi_{21}+\nabla \Phi_{22}$ (см. (1.1.20)).

Из (1.5.9) следует, что $C$ - самосопряженный неотрицательный оператор. Покажем, что он положительно определен. Из представления (1.5.8) и свойств операторов $V$ и $\gamma_{n, 1}$ (см. леммы 2.3 и 1.2) следует, что имеет место неравенство

$$
\left\|\Phi_{22}\right\|_{H_{\Omega_{2}}^{1}\left(\Omega_{2} ; \rho_{2,0}\right)}=\left(\left.\int_{\Omega_{2}} \rho_{2,0} \nabla \Phi_{22}\right|^{2} d \Omega_{2}\right)^{1 / 2} \leqslant c\left\|\boldsymbol{u}_{1}\right\|_{J_{0, S_{1}}\left(\Omega_{1}\right)}, \quad c>0 .
$$

Отсюда при $\alpha+\beta=1, \alpha, \beta>0$, получаем что

$$
\begin{aligned}
\rho_{1} \int_{\Omega_{1}}\left|\boldsymbol{u}_{1}\right|^{2} d \Omega_{1} & +\int_{\Omega_{2}} \rho_{2,0}\left|\nabla \Phi_{21}+\nabla \Phi_{22}\right|^{2} d \Omega_{2} \geqslant \rho_{1} \alpha\left\|\boldsymbol{u}_{1}\right\|_{\boldsymbol{J}_{0, S_{1}}\left(\Omega_{1}\right)}^{+} \\
& +\rho_{1} \beta c^{-2} \int_{\Omega_{2}} \rho_{2,0}\left|\nabla \Phi_{22}\right|^{2} d \Omega_{2}+\int_{\Omega_{2}} \rho_{2,0}\left|\nabla \Phi_{22}\right|^{2} d \Omega_{2}+\int_{\Omega_{2}}\left|\nabla \Phi_{21}\right|^{2} d \Omega_{2}- \\
& -\varepsilon \int_{\Omega_{2}} \rho_{2,0}\left|\nabla \Phi_{21}\right|^{2} d \Omega_{2}-\varepsilon^{-1} \int_{\Omega_{2}} \rho_{2,0}\left|\nabla \Phi_{22}\right|^{2} d \Omega_{2} \geqslant \rho_{1} \alpha\left\|\boldsymbol{u}_{1}\right\|_{\boldsymbol{J}_{0, S_{1}}\left(\Omega_{1}\right)^{+}}+ \\
& +\left(\rho_{1} \beta c^{-2}+1-\varepsilon^{-1}\right) \int_{\Omega_{2}} \rho_{2,0}\left|\nabla \Phi_{22}\right|^{2} d \Omega_{2}+(1-\varepsilon) \int_{\Omega_{2}}\left|\nabla \Phi_{21}\right|^{2} d \Omega_{2}, \quad \varepsilon>0 .
\end{aligned}
$$

Выбирая $\varepsilon$ так, чтобы было выполнено условие

$$
\left(1+\rho_{1} \beta c^{-2}\right)^{-1} \leqslant \varepsilon<1,
$$

приходим к выводу, что квадратичная форма в левой части (1.5.10) положительно определена в пространстве $\boldsymbol{J}_{0, S_{1}}\left(\Omega_{1}\right) \oplus \boldsymbol{G}\left(\Omega_{2} ; \rho_{2,0}\right)$. Отсюда и из (1.5.9) следует, что оператор $C$ положительно определен в $\mathcal{H}=\boldsymbol{J}_{0, S_{1}}\left(\Omega_{1}\right) \oplus L_{2, \Gamma} \oplus \boldsymbol{G}\left(\Omega_{2} ; \rho_{2,0}\right) \oplus L_{2}\left(\Omega_{2} ; \rho_{2,0}^{-1}\right)$.

1.6. Дальнейшие преобразования задачи. Изучив свойства оператора полной энергии $C$ из (1.5.3), продолжим рассмотрение этой задачи и напомним, что оператор $B$ из (1.5.3) содержит интегральный оператор Вольтерра $I_{01}(t)$ (см. (1.1.4)), отвечающий обобщенной модели Олдройта. Это позволяет перейти от (1.5.3) к дифференциальному уравнению в гильбертовом пространстве, введя новые искомые функции в изучаемой задаче.

Проделаем соответствующие преобразования для простоты при $m=2$ (для других $m \in \mathbb{N}$ преобразования аналогичные). Именно, введем функции

$$
\boldsymbol{w}_{k}(t, x):=\mu_{1}^{1 / 2} \alpha_{k} A_{1}^{1 / 2} \int_{0}^{t} e^{-\beta_{k}(t-s)} \boldsymbol{u}_{1}(s, x) d s, \quad \boldsymbol{w}_{k}(0, x)=\mathbf{0}, \quad k=1,2 .
$$

Отсюда имеем

$$
\frac{d \boldsymbol{w}_{k}}{d t}=\mu_{1}^{1 / 2} \alpha_{k}^{1 / 2} A_{1}^{1 / 2} \boldsymbol{u}_{1}-\beta_{k} \boldsymbol{w}_{k}, \quad k=1,2 .
$$

Введем также новый искомый вектор-столбец

$$
\tilde{z}(t):=\left(z_{1}^{\tau} ; z_{2}^{\tau}\right)^{\tau}, \quad z_{1}^{\tau}=\left(\boldsymbol{u}_{1} ; \boldsymbol{w}_{1} ; \boldsymbol{w}_{2} ; \zeta\right), \quad z_{2}^{\tau}=\left(\nabla \Phi_{21} ; p_{2}\right) .
$$


Тогда задача (1.5.3) перейдет с учетом (1.6.2) в задачу Коши для системы дифференциальных уравнений первого порядка следующего вида:

$$
\tilde{C} \frac{d \tilde{z}}{d t}+\tilde{B} \tilde{z}=\tilde{f}, \quad \tilde{z}(0)=\tilde{z}^{0}
$$

где

$$
\begin{gathered}
\tilde{C}:=\left(\begin{array}{cc}
\tilde{C}_{11} & \tilde{C}_{12} \\
\tilde{C}_{21} & \tilde{C}_{22}
\end{array}\right), \quad B:=\left(\begin{array}{cc}
B_{11} & 0 \\
0 & B_{22}
\end{array}\right), \\
\tilde{f}=\left(\rho_{1} P_{0, S_{1}} \boldsymbol{f}_{1}-\rho_{2,0}(0) Q P_{\Gamma} \gamma_{2} F_{2} ; \mathbf{0} ; \mathbf{0} ; 0 ; \nabla F_{2} ; 0\right)^{\tau}, \\
\tilde{C}_{11}:=\operatorname{diag}\left(\rho_{1} \tilde{I}_{1}+\left(\rho_{2,0}(0)\right)^{2}\left(Q \gamma_{2}\right)\left(V \gamma_{n, 1}\right) ; \tilde{I}_{2} ; \tilde{I}_{3} ; g\left(\rho_{1}-\rho_{2,0}(0)\right) \tilde{I}_{4}\right) ; \\
\tilde{C}_{22}:=\operatorname{diag}\left(\tilde{I}_{5} ; a^{-2} \tilde{I}_{6}\right) ;
\end{gathered}
$$

здесь $\tilde{I}_{k}$ - единичные операторы в пространствах элементов из (1.6.3). Далее,

$$
\begin{aligned}
\tilde{C}_{12} & :=\left(\begin{array}{ccc}
-\rho_{2,0}(0) Q P_{\Gamma} \gamma_{2} \nabla^{-1} & 0 \\
0 & 0 \\
0 & 0 \\
0 & 0
\end{array}\right), \quad \tilde{C}_{21}:=\left(\begin{array}{cccc}
-\rho_{2,0}(0) \nabla V \gamma_{n, 1} & 0 & 0 & 0 \\
0 & 0 & 0 & 0
\end{array}\right), \\
B_{11} & :=\left(\begin{array}{cccc}
\mu_{1} A_{1} & \mu_{1}^{1 / 2} \alpha_{1}^{1 / 2} A_{1}^{1 / 2} & \mu_{1}^{1 / 2} \alpha_{2}^{1 / 2} A_{1}^{1 / 2} & g\left(\rho_{1}-\rho_{2,0}(0)\right) Q \\
-\mu_{1}^{1 / 2} \alpha_{1}^{1 / 2} A_{1}^{1 / 2} & \beta_{1} \tilde{I}_{2} & 0 & 0 \\
-\mu_{1}^{1 / 2} \alpha_{2}^{1 / 2} A_{1}^{1 / 2} & 0 & \beta_{2} \tilde{I}_{3} & 0 \\
-g\left(\rho_{1}-\rho_{2,0}(0)\right) \gamma_{n, 1} & 0 & 0 & 0
\end{array}\right), \\
B_{22} & :=\left(\begin{array}{cc}
0 & B_{34} \\
B_{43} & 0
\end{array}\right) .
\end{aligned}
$$

Заметим, что искомые элементы $\tilde{z}(t)$ теперь считаются функциями переменной $t$ со значениями в пространстве

$$
\tilde{\mathcal{H}}=\left(\boldsymbol{J}_{0, S_{1}}\left(\Omega_{1}\right) \oplus \boldsymbol{J}_{0, S_{1}}\left(\Omega_{1}\right) \oplus \boldsymbol{J}_{0, S_{1}}\left(\Omega_{1}\right) \oplus L_{2, \Gamma}\right) \oplus\left(\boldsymbol{G}\left(\Omega_{2} ; \rho_{2,0}\right) \oplus L_{2}\left(\Omega_{2} ; \rho_{2,0}^{-1}\right)\right)=: \tilde{\mathcal{H}}_{1} \oplus \tilde{\mathcal{H}}_{2}
$$

(см. (1.5.1)).

Дальнейший план исследований - изучить свойства операторных матриц $\tilde{C}$ и $\tilde{B}$ в (1.6.4) и доказать теорему существования сильного по переменной $t$ решения этой задачи.

Отметим предварительно следующий факт.

Лемма 1.6. Оператор $\tilde{C}: \tilde{\mathcal{H}} \rightarrow \tilde{\mathcal{H}}$ является ограниченным положительно определенным оператором, действующим в $\tilde{\mathcal{H}}$.

Доказательство. Лемма 1.6 непосредственно следует из леммы 1.5 с учетом вида операторной матрицы $\tilde{C}_{11}$, в частности, с учетом добавления единичных элементов $\tilde{I}_{2}, \tilde{I}_{3}$ на диагонали.

Изучим теперь свойства операторных блоков $B_{11}$ и $B_{22}$ в операторной матрице $\tilde{B}$ (см. (1.6.6)).

Лемма 1.7. Операторная матрица $B_{11}$ заданная на области определения

$$
\mathcal{D}\left(B_{11}\right)=\mathcal{D}\left(A_{1}\right) \oplus \mathcal{D}\left(A_{1}\right) \oplus \mathcal{D}\left(A_{1}\right) \oplus H_{\Gamma}^{1 / 2},
$$

плотной в пространстве

$$
\tilde{\mathcal{H}}_{1}=\boldsymbol{J}_{0, S_{1}}\left(\Omega_{1}\right) \oplus \boldsymbol{J}_{0, S_{1}}\left(\Omega_{1}\right) \oplus \boldsymbol{J}_{0, S_{1}}\left(\Omega_{1}\right) \oplus L_{2, \Gamma},
$$

допускает факторизацию с симметричными крайними множителями:

$$
B_{11}=\operatorname{diag}\left(\mu_{1}^{1 / 2} A_{1}^{1 / 2} ; \tilde{I}_{2} ; \quad \tilde{I}_{3} ; \tilde{I}_{4}\right) \cdot E_{0} \cdot \operatorname{diag}\left(\mu_{1}^{1 / 2} A_{1}^{1 / 2} ; \quad \tilde{I}_{2} ; \quad \tilde{I}_{3} ; \tilde{I}_{4}\right),
$$


где

$$
E_{0}:=\left(\begin{array}{cccc}
\tilde{I}_{1} & \alpha_{1}^{1 / 2} \tilde{I}_{2} & \alpha_{2}^{1 / 2} \tilde{I}_{3} & g \mu_{1}^{-1 / 2}\left(\rho_{1}-\rho_{2,0}(0)\right) A_{1}^{-1 / 2} Q \\
-\alpha_{1}^{1 / 2} \tilde{I}_{1} & \beta_{1} \tilde{I}_{2} & 0 & 0 \\
-\alpha_{2}^{1 / 2} \tilde{I}_{1} & 0 & \beta_{2} \tilde{I}_{3} & 0 \\
-g \mu_{1}^{-1 / 2}\left(\rho_{1}-\rho_{2,0}(0)\right) \gamma_{n, 1} A_{1}^{-1 / 2} & 0 & 0 & 0
\end{array}\right) .
$$

Эта операторная матрица является аккретивным оператором:

$$
\operatorname{Re}\left(B_{11} z_{1}, z_{1}\right)_{\tilde{\mathcal{H}}_{1}} \geqslant 0 \quad \forall z_{1} \in \mathcal{D}\left(B_{11}\right) .
$$

При любом $b>0$ операторная матрица

$$
B_{11, b}:=B_{11}+b P_{4}, \quad P_{4}:=\operatorname{diag}\left(0 ; 0 ; 0 ; \tilde{I}_{4}\right)
$$

является равномерно аккретивным оператором:

$$
\operatorname{Re}\left(B_{11, b} z_{1}, z_{1}\right)_{\tilde{\mathcal{H}}_{1}} \geqslant c\left\|z_{1}\right\|_{\tilde{\mathcal{H}}_{1}}^{2}, \quad c>0, \quad \forall z_{1} \in \mathcal{D}\left(B_{11, b}\right)=\mathcal{D}\left(B_{11}\right) .
$$

Доказательство. Факторизация (1.6.7) матрицы $B_{11}$ проверяется непосредственно. Свойство аккретивности (1.6.9) следует из равенства

$$
\operatorname{Re}\left(B_{11} z_{1}, z_{1}\right)_{\tilde{\mathcal{H}}_{1}}=\mu_{1}\left\|A_{1}^{1 / 2} \boldsymbol{u}_{1}\right\|_{\boldsymbol{J}_{0, S_{1}}\left(\Omega_{1}\right)}^{2}+\sum_{k=1}^{2} \beta_{k}\left\|\boldsymbol{w}_{k}\right\|_{\boldsymbol{J}_{0, S_{1}}\left(\Omega_{1}\right)}^{2} .
$$

Отсюда получаем свойство равномерной аккретивности (1.6.11), если заметить, что

$$
\left\|A_{1}^{1 / 2} \boldsymbol{u}_{1}\right\|_{\boldsymbol{J}_{0, S_{1}}\left(\Omega_{1}\right)}^{2} \geqslant \lambda_{1}\left(A_{1}\right)\left\|\boldsymbol{u}_{1}\right\|_{\boldsymbol{J}_{0, S_{1}}\left(\Omega_{1}\right)}^{2}
$$

где $\lambda_{1}\left(A_{1}\right)>0$ - первое собственное значение оператора $A_{1}$. Тогда в (1.6.11) имеем

$$
c=\max \left\{\mu_{1} \lambda_{1}\left(A_{1}\right) ; \beta_{1}, \beta_{2} ; b\right\}>0 .
$$

Лемма 1.8. Оператор $\gamma_{n, 1} A_{1}^{-1 / 2}: \boldsymbol{J}_{0, S_{1}}\left(\Omega_{1}\right) \rightarrow L_{2, \Gamma}$ компактен. Оператор $A_{1}^{-1 / 2} Q: \mathcal{D}(Q) \rightarrow$ $\boldsymbol{J}_{0, S_{1}}\left(\Omega_{1}\right)$ и его замыкание обладают свойствами

$$
A_{1}^{-1 / 2} Q=\left.\left(\gamma_{n, 1} A_{1}^{-1 / 2}\right)^{*}\right|_{\mathcal{D}(Q)}, \quad \overline{A_{1}^{-1 / 2} Q}=\left(\gamma_{n, 1} A_{1}^{-1 / 2}\right)^{*} .
$$

Доказательство. Оператор $A_{1}^{-1 / 2}$ переводит $\boldsymbol{J}_{0, S_{1}}\left(\Omega_{1}\right)$ в $\boldsymbol{J}_{0, S_{1}}^{1}\left(\Omega_{1}\right) \subset \boldsymbol{H}^{1}\left(\Omega_{1}\right)$, а тогда (по теореме Гальярдо, см. [21]) $\gamma_{n, 1} A_{1}^{-1 / 2}$ ограниченно действует из $\boldsymbol{J}_{0, S_{1}}\left(\Omega_{1}\right)$ в $H_{\Gamma}^{1 / 2}=H^{1 / 2}(\Gamma) \cap L_{2, \Gamma}$. Так как $H_{\Gamma}^{1 / 2}$ компактно вложено в $L_{2, \Gamma}$, то $\gamma_{n, 1} A_{1}^{-1 / 2}$ - компактный оператор.

Далее, пусть $\boldsymbol{u}_{1}$ - произвольный элемент из $\boldsymbol{J}_{0, S_{1}}\left(\Omega_{1}\right)$ и $\zeta \in \mathcal{D}(Q) \subset L_{2, \Gamma}$. Тогда

$$
\left(\gamma_{n, 1} A_{1}^{-1 / 2} \boldsymbol{u}_{1}, \zeta\right)_{L_{2, \Gamma}}=\left(A_{1}^{-1 / 2} \boldsymbol{u}_{1}, Q \zeta\right)_{\boldsymbol{J}_{0, S_{1}}\left(\Omega_{1}\right)}=\left(\boldsymbol{u}_{1}, A_{1}^{-1 / 2} Q \zeta\right)_{\boldsymbol{J}_{0, S_{1}}\left(\Omega_{1}\right)}
$$

Отсюда следует, что $A_{1}^{-1 / 2} Q=\left.\left(\gamma_{n, 1} A_{1}^{-1 / 2}\right)^{*}\right|_{\mathcal{D}(Q)}$, и так как $\mathcal{D}(Q) \subset H_{\Gamma}^{1 / 2}$ плотно в $L_{2, \Gamma}$, то имеет место свойство (1.6.12), причем $\overline{A_{1}^{-1 / 2} Q}$ - компактный оператор.

Заметим теперь, что оператор $B_{11, b}$ из (1.6.10) также допускает факторизацию вида (1.6.7) с теми же крайними множителями и со средним множителем

$$
E_{b}:=\left(\begin{array}{cccc}
\tilde{I}_{1} & \alpha_{1}^{1 / 2} \tilde{I}_{2} & \alpha_{2}^{1 / 2} \tilde{I}_{3} & g \mu_{1}^{-1 / 2}\left(\rho_{1}-\rho_{2,0}(0)\right) A_{1}^{-1 / 2} Q \\
-\alpha_{1}^{1 / 2} \tilde{I}_{1} & \beta_{1} \tilde{I}_{2} & 0 & 0 \\
-\alpha_{2}^{1 / 2} \tilde{I}_{1} & 0 & \beta_{2} \tilde{I}_{3} & 0 \\
-g \mu_{1}^{-1 / 2}\left(\rho_{1}-\rho_{2,0}(0)\right) \gamma_{n, 1} A_{1}^{-1 / 2} & 0 & 0 & b \tilde{I}_{4}
\end{array}\right) .
$$

Из приведенных фактов вытекает следующее утверждение. 
Лемма 1.9. Оператор $B_{11, b}$ из (1.6.10) допускает расширение путем замыкания среднего множителя (1.6.13) на основе (1.6.12). При этом замыкание $\overline{B_{11, b}}$ имеет факторизацию

$$
\overline{B_{11, b}}=\operatorname{diag}\left(\mu_{1}^{1 / 2} A_{1}^{1 / 2} ; \tilde{I}_{2} ; \tilde{I}_{3} ; \tilde{I}_{4}\right) \cdot \overline{E_{b}} \cdot \operatorname{diag}\left(\mu_{1}^{1 / 2} A_{1}^{1 / 2} ; \tilde{I}_{2} ; \tilde{I}_{3} ; \tilde{I}_{4}\right),
$$

является максимальным равномерно аккретивным оператором в $\tilde{\mathcal{H}}_{1}$, заданным на области определения

$$
\begin{aligned}
\mathcal{D}\left(\overline{B_{11, b}}\right)=\left\{z_{1}=\right. & \left(\boldsymbol{u}_{1} ; \boldsymbol{w}_{1} ; \boldsymbol{w}_{2} ; \zeta\right)^{\tau}: \\
& \left.\mu_{1}^{1 / 2} A_{1}^{1 / 2} \boldsymbol{u}_{1}+\sum_{k=1}^{2} \alpha_{k}^{1 / 2} \boldsymbol{w}_{k}+g \mu_{1}^{-1 / 2}\left(\rho_{1}-\rho_{2,0}(0)\right)\left(\gamma_{n, 1} Q\right)^{*} \zeta \in \mathcal{D}\left(A_{1}^{1 / 2}\right)\right\}
\end{aligned}
$$

и действует по закону

$$
\overline{B_{11, b}} z_{1}=\left(\begin{array}{c}
\mu_{1}^{1 / 2} A_{1}^{1 / 2}\left(\mu_{1}^{1 / 2} A_{1}^{1 / 2} \boldsymbol{u}_{1}+\sum_{k=1}^{2} \alpha_{k}^{1 / 2} \boldsymbol{w}_{k}+g \mu_{1}^{-1 / 2}\left(\rho_{1}-\rho_{2,0}(0)\right)\left(\gamma_{n}, 1 A^{-1 / 2}\right)^{*} \zeta\right) \\
-\mu_{1}^{1 / 2} \alpha_{1}^{1 / 2} A_{1}^{1 / 2} \boldsymbol{u}_{1}+\beta_{1} \boldsymbol{w}_{1} \\
-\mu_{1}^{1 / 2} \alpha_{2}^{1 / 2} A_{1}^{1 / 2} \boldsymbol{u}_{1}+\beta_{2} \boldsymbol{w}_{2} \\
-g \mu_{1}^{-1 / 2}\left(\rho_{1}-\rho_{2,0}(0)\right) \gamma_{n, 1} \boldsymbol{u}_{1}+b \zeta
\end{array}\right) .
$$

Доказательство. В доказательстве нуждается лишь свойство максимальности оператора $\overline{B_{11, b}}$. Однако этот факт следует из того, что в представлении (1.6.14) каждая операторная матрица ограниченно обратима в $\tilde{\mathcal{H}}_{1}$, в частности, для $\overline{E_{b}}$ это следует из того факта, что неравенство (1.6.11) сохраняется и для $\overline{B_{11, b}}$, и потому область значений $\mathcal{R}\left(\overline{B_{11, b}}\right)$ есть все пространство $\tilde{\mathcal{H}}_{1}$.

Из леммы 1.9 получаем следующий важный вывод.

Лемма 1.10. Оператор - $\overline{B_{11, b}}$ является генератором сжимающей полугруппы $U_{1}(t)$, причем $\left\|U_{1}(t)\right\| \leqslant e^{-c t}, c>0$ (см. (1.6.11), $\left.(\square)\right)$. При этом оператор $-\overline{B_{11}}:=-\left(\overline{B_{11, b}}-b P_{4}\right)$ такэе является генератором сжимающей полугруппы операторов.

Перейдем теперь к изучению свойств оператора $B_{22}$ из (1.6.6), отвечающего движению газа в исследуемой системе. Этот оператор действует в пространстве $\tilde{\mathcal{H}}_{2}=\boldsymbol{G}\left(\Omega_{2} ; \rho_{2,0}\right) \oplus L_{2}\left(\Omega_{2} ; \rho_{2,0}^{-1}\right)$ с элементами $z_{2}=\left(\nabla \Phi_{21} ; p_{2}\right)^{\tau}$. Очевидно, это оператор неограничен, и его область значений должна совпадать с $\tilde{\mathcal{H}}_{2}$. Поэтому далее будем считать, что

$$
\begin{array}{r}
\mathcal{D}\left(B_{22}\right)=\left\{\nabla \Phi_{21} \in \boldsymbol{G}\left(\Omega_{2} ; \rho_{2,0}\right): \operatorname{div}\left(\rho_{2,0} \nabla \Phi_{21}\right) \in L_{2}\left(\Omega_{2} ; \rho_{2,0}^{-1}\right), \rho_{2,0} \frac{\partial \Phi_{21}}{\partial n}=0 \text { на } \partial \Omega_{2}\right\} \oplus \\
\oplus\left\{p_{2} \in L_{2}\left(\Omega_{2} ; \rho_{2,0}^{-1}\right): \nabla\left(\rho_{2,0}^{-1} p_{2}\right) \in \boldsymbol{G}\left(\Omega_{2} ; \rho_{2,0}\right)\right\} .
\end{array}
$$

Лемма 1.11. Оператор $B_{22}: \mathcal{D}\left(B_{22}\right) \subset \tilde{\mathcal{H}}_{2} \rightarrow \tilde{\mathcal{H}}_{2}$ является кососамосопряљсенным, т.е.

$$
B_{22}^{*}=-B_{22} \text {. }
$$

Доказательство. Пусть $z_{2}=\left(\nabla \Phi_{21} ; p_{2}\right)^{\tau} \in \mathcal{D}\left(B_{22}\right), y_{2}=\left(\nabla \Psi_{21} ; q_{2}\right)^{\tau} \in \mathcal{D}\left(B_{22}\right)$. Тогда, используя формулу вида $\operatorname{div}(\varphi \boldsymbol{A})=\varphi \operatorname{div} \boldsymbol{A}+\nabla \varphi \cdot \boldsymbol{A}$, вычислим выражение

$$
\begin{aligned}
\left(B_{22} z_{2}, y_{2}\right)_{\tilde{\mathcal{H}}_{2}}= & \int_{\Omega_{2}} \rho_{2,0} \nabla \Phi_{21} \cdot \overline{\nabla \Psi_{21}} d \Omega_{2}+\int_{\Omega_{2}} \rho_{2,0}^{-1} \operatorname{div}\left(\rho_{2,0} \nabla \Phi_{21}\right) \overline{q_{2}} d \Omega_{2}= \\
& =\left\{\int_{\Omega_{2}} \operatorname{div}\left(\rho_{2,0}^{-1} p_{2} \rho_{2,0} \overline{\nabla \Psi_{21}}\right) d \Omega_{2}-\int_{\Omega_{2}} \rho_{2,0}^{-1} p_{2} \operatorname{div}\left(\rho_{2,0} \overline{\nabla \Psi_{21}}\right) d \Omega_{2}\right\}+
\end{aligned}
$$




$$
\begin{aligned}
& +\left\{\int_{\Omega_{2}} \operatorname{div}\left(\rho_{2,0}^{-1} \overline{q_{2}} \rho_{2,0} \nabla \Phi_{21}\right) d \Omega_{2}-\int_{\Omega_{2}} \rho_{2,0} \nabla \Phi_{21} \cdot \nabla\left(\rho_{2,0}^{-1} \overline{q_{2}}\right) d \Omega_{2}\right\}= \\
& =-\left[\int_{\Omega_{2}} \rho_{2,0} \nabla \Phi_{21} \cdot \overline{\nabla\left(\rho_{2,0}^{-1} q_{2}\right)} d \Omega_{2}+\int_{\Omega_{2}} \rho_{2,0}^{-1} p_{2} \overline{\operatorname{div}\left(\rho_{2,0} \nabla \Psi_{21}\right)} d \Omega_{2}\right]=-\left(z_{2}, B_{22} y_{2}\right)_{\tilde{\mathcal{H}}_{2}}
\end{aligned}
$$

для всех $z_{2}, y_{2} \in \mathcal{D}\left(B_{22}\right)$ (здесь при выводе использовано граничное условие на $\partial \Omega_{2}$, см. (1.6.16), и теорема Гаусса-Остроградского). Отсюда и следует свойство (1.6.17).

Рассмотрим подробнее другие свойства оператора $B_{22}$ и его связь с оператором задачи Неймана (1.3.5).

Лемма 1.12. Oператор $B_{22}: \mathcal{D}\left(B_{22}\right) \subset \tilde{\mathcal{H}}_{2} \rightarrow \tilde{\mathcal{H}}_{2}$ обратим.

Доказательство. Рассмотрим уравнение $B_{22} z_{2}=y_{2} \in \tilde{\mathcal{H}}_{2}, z_{2}=\left(\nabla \Phi_{21} ; p_{2}\right)^{\tau}$, причем $y_{2}$ представим в виде $y_{2}=\left(\nabla\left(\rho_{2,0}^{-1} \psi_{2}\right) ; \rho_{2,0} q_{2}\right)^{\tau}$, с заданными произвольными функциями $\psi_{2}$ и $q_{2}$. Тогда будем иметь соотношения

$$
\nabla\left(\rho_{2,0}^{-1} p_{2}\right)=\nabla\left(\rho_{2,0}^{-1} \psi_{2}\right)
$$

и задачу

$$
\operatorname{div}\left(\rho_{2,0} \nabla \Phi_{21}\right)=\rho_{2,0} q_{2} \quad \text { в } \Omega_{2}, \quad \rho_{2,0} \frac{\partial \Phi_{21}}{\partial n}=0 \quad \text { на } \partial \Omega_{2} .
$$

Отсюда в силу нормировки

$$
\int_{\Omega_{2}} \rho_{2,0}\left(\rho_{2,0}^{-1} \psi_{2}\right) d \Omega_{2}=0
$$

(см. (1.2.9)) для элементов из $\boldsymbol{G}\left(\Omega_{2} ; \rho_{2,0}\right)$ получаем $p_{2}=\psi_{2}$, а в задаче (1.6.18) возникает проблема

$$
\begin{aligned}
& -\Delta_{0} \Phi_{21}=-\rho_{2,0}^{-1} \operatorname{div}\left(\rho_{2,0} \nabla \Phi_{21}\right)=-q_{2} \quad \text { в } \Omega_{2}, \\
& \rho_{2,0} \frac{\partial \Phi_{21}}{\partial n}=0 \quad \text { на } \partial \Omega_{2}, \quad \int_{\Omega_{2}} \rho_{2,0} \Phi_{21} d \Omega_{2}=0
\end{aligned}
$$

(см. (1.3.8)), равносильная уравнению

$$
A_{2} \Phi_{21}=-q_{2} \in L_{2, \Omega_{2}, \rho_{2,0}}
$$

(см. (1.3.7)), где $A_{2}$-оператор гильбертовой пары $\left(H_{\Omega_{2}}^{1}\left(\Omega_{2} ; \rho_{2,0}\right) ; L_{2, \Omega_{2}, \rho_{2,0}}\right)$, изученный в п. 1.3 . Отсюда следует, что задача (1.6.19) однозначно разрешима: $\Phi_{21}=-A_{2}^{-1} q_{2} \in H_{\Omega_{2}}^{1}\left(\Omega_{2} ; \rho_{2,0}\right)$ и потому $\nabla \Phi_{21} \in \boldsymbol{G}\left(\Omega_{2} ; \rho_{2,0}\right)$ (между элементами из $\boldsymbol{G}\left(\Omega_{2} ; \rho_{2,0}\right)$ и $H_{\Omega_{2}}^{1}\left(\Omega_{2} ; \rho_{2,0}\right)$ имеется изоморфизм, осуществляемый операторами $\nabla$ и $\left.\nabla^{-1}\right)$.

Лемма 1.13. Спектр оператора $B_{22}$ дискретен и расположен на мнимой оси. Его собственные значения образуют множество $\left\{\lambda_{k}^{ \pm}\left(B_{22}\right)\right\}_{k=1}^{\infty}$ с двумя ветвями:

$$
\lambda_{k}^{ \pm}\left(B_{22}\right)= \pm i \lambda_{k}^{1 / 2}\left(A_{2}\right), \quad k=1,2, \ldots,
$$

где $\left\{\lambda_{k}\left(A_{2}\right)\right\}_{k=1}^{\infty}$-собственные значения оператора $A_{2}$ (см. (1.6.19)):

$$
\begin{gathered}
0<\lambda_{1}\left(A_{2}\right) \leqslant \lambda_{2}\left(A_{2}\right) \leqslant \ldots \leqslant \lambda_{k}\left(A_{2}\right) \leqslant \ldots, \\
\lambda_{k}\left(A_{2}\right)=\left(\frac{\left|\Omega_{2}\right|}{6 \pi^{2}}\right)^{-2 / 3} k^{2 / 3}[1+o(1)], \quad k \rightarrow \infty .
\end{gathered}
$$

Собственные элементы

$$
\left\{z_{k}^{ \pm}\right\}_{k=1}^{\infty}=\left\{\left(\nabla \Phi_{21, k}^{ \pm} ; p_{2, k}^{ \pm}\right)^{\tau}\right\}_{k=1}^{\infty}
$$


отвечающие собственным значениям оператора $B_{22}$, образуют ортогональный базис в $\tilde{\mathcal{H}}_{2}$ и въраэсаются через ортонормированные собственные элементы оператора $A_{2}$, т.е. решения спектральной задачи

$$
-\Delta_{0} \Phi_{21}=\lambda \Phi_{21} \quad{ } \Omega_{2}, \quad \rho_{2,0} \frac{\partial \Phi_{21}}{\partial n}=0 \quad \text { на } \partial \Omega_{2}, \quad \int_{\Omega_{2}} \rho_{2,0} \Phi_{21} d \Omega_{2}=0,
$$

по формулам

$$
z_{k}^{ \pm}=\left(\nabla \Phi_{21, k} ; \lambda_{k}^{ \pm} \rho_{2,0} \Phi_{21, k}\right)^{\tau}, \quad k=1,2, \ldots
$$

При этом для ортонормированных в $L_{2, \Omega_{2}, \rho_{2,0}}$ собственных элементов задачи (1.6.21) выполнены следующие формулы ортогональности для ортонормированных элементов $\left\{z_{k}^{ \pm}\right\}_{k=1}^{\infty}$ :

$$
\left(z_{k}^{ \pm}, z_{l}^{ \pm}\right)_{\tilde{\mathcal{H}}_{2}}=\left(\frac{1+( \pm 1)( \pm 1)}{2}\right)^{1 / 2} \delta_{k l}
$$

где учтены все четыре варианта знаков слева.

Доказательство проводится непосредственной проверкой всех связей возникающих задач для операторов $B_{22}$ и $A_{2}$. Асимптотическая формула (1.6.20) - это известная асимптотика Вейля в спектральной задаче Неймана для оператора Лапласа при $\rho_{2,0}=$ const.

Подводя итоги исследования свойств оператора $\tilde{B}=\operatorname{diag}\left(B_{11}, B_{22}\right)$ в задаче Коши (1.6.4), отметим, что первый блок $B_{11}$ характеризует диссипативную вязкоупругую часть исследуемой гидросистемы (жидкость), а второй блок $B_{22}$ - консервативную часть (газ). В итоге приходим к следующему выводу.

Лемма 1.14. После замыкания (оператора $B_{11}$ ) оператор $\overline{\tilde{B}}$ является максималъным аккретивным оператором, действующим в $\tilde{\mathcal{H}}=\tilde{\mathcal{H}}_{1} \oplus \tilde{\mathcal{H}}_{2}$ и заданным на области определения

$$
\mathcal{D}(\overline{\tilde{B}})=\mathcal{D}\left(\overline{\tilde{B}}_{11}\right) \oplus \mathcal{D}\left(B_{22}\right), \quad \mathcal{D}\left(\bar{B}_{11}\right)=\mathcal{D}\left(\bar{B}_{11, b}\right), \quad \mathcal{R}(\overline{\tilde{B}})=\tilde{\mathcal{H}} .
$$

Доказательство. Так как $B_{22}$ кососамосопряжен в $\tilde{\mathcal{H}}_{2}$, то он является генератором группы унитарных операторов, действующих в $\tilde{\mathcal{H}}_{2}$. Поэтому вместе с утверждениями лемм 1.9 и 1.10 получаем утверждение леммы 1.14.

1.7. О разрешимости исходной начально-краевой задачи. Опираясь на установленные факты, докажем утверждение о разрешимости задачи Коши (1.5.3), к которой была приведена исходная начально-краевая задача (1.1.5)-(1.1.13).

Определение 1.1. Будем говорить, что задача Коши (1.5.3) имеет сильное по переменной $t$ решение $z(t)=\left(\boldsymbol{u}_{1} ; \zeta ; \nabla \Phi_{21} ; p_{2}\right)^{\tau}$ на отрезке $[0, T]$, если все слагаемые в уравнении (1.5.3) являются непрерывными функциями $t$ со значениями в пространстве

$$
\mathcal{H}:=\boldsymbol{J}_{0, S_{1}}\left(\Omega_{1}\right) \oplus L_{2, \Gamma} \oplus \boldsymbol{G}\left(\Omega_{2} ; \rho_{2,0}\right) \oplus L_{2}\left(\Omega_{2} ; \rho_{2,0}^{-1}\right)
$$

и выполнено начальное условие $z(0)=z^{0}$.

Согласно этому определению для сильного решения задачи все слагаемые в уравнении (1.4.13) должны быть элементами из $C\left([0, T] ; \boldsymbol{J}_{0, S_{1}}\left(\Omega_{1}\right)\right)$, в уравнении $(1.4 .14)$ - элементами из $C\left([0, T] ; L_{2, \Gamma}\right)$, в уравнении $(1.4 .15)$ - элементами из $C\left([0, T] ; \boldsymbol{G}\left(\Omega_{2} ; \rho_{2,0}\right)\right)$, а в уравнении (1.4.16) - элементами из $C\left([0, T] ; L_{2}\left(\Omega_{2} ; \rho_{2,0}^{-1}\right)\right)$.

Теорема 1.1. Пусть в задаче (1.5.3) выполнены условия

$$
\begin{gathered}
\boldsymbol{u}_{1}^{0} \in \mathcal{D}\left(A_{1}\right) \subset \mathcal{D}\left(A_{1}^{1 / 2}\right)=\boldsymbol{J}_{0, S_{1}}^{1}\left(\Omega_{1}\right), \quad \zeta^{0} \in \mathcal{D}(Q)=H_{\Gamma}^{1 / 2}, \\
\Phi_{21}^{0} \in \mathcal{D}\left(A_{2}\right) \subset \mathcal{D}\left(A_{2}^{1 / 2}\right)=H_{\Omega_{2}}^{1}\left(\Omega_{2} ; \rho_{2,0}\right), \quad \nabla\left(\rho_{2,0}^{-1} p_{2}^{0}\right) \in \boldsymbol{G}\left(\Omega_{2} ; \rho_{2,0}\right),
\end{gathered}
$$

а также условия

$$
\boldsymbol{f}_{1}(t, x) \in C^{1}\left([0, T] ; \boldsymbol{L}_{2}\left(\Omega_{1}\right)\right), \quad \boldsymbol{f}_{2}(t, x) \in C^{1}\left([0, T] ; \boldsymbol{L}_{2}\left(\Omega_{2}\right)\right) .
$$


Тогда задача (1.5.3) имеет сильное по переменной $t$ решение $z(t)$ на отрезке $[0, T]$ (в смысле определения 1.1).

Доказательство состоит из нескольких этапов.

1. Рассмотрим задачу Коши вида (1.6.4), однако с оператором $\overline{\tilde{B}}=\operatorname{diag}\left(\overline{B_{11}} ; B_{22}\right)$, который, согласно леммам 1.9-1.11, 1.14, является максимальным аккретивным:

$$
\tilde{C} \frac{d \tilde{z}}{d t}+\tilde{\tilde{B}} \tilde{z}=\tilde{f}(t), \quad \tilde{z}(0)=\tilde{z}^{0}
$$

При этом начальные данные выберем в виде (1.7.1), т.е. из области определения оператора $B$ (см. (1.5.3)), и дополним их тривиальными начальными условиями

$$
\boldsymbol{w}_{k}(0)=\mathbf{0}, \quad k=1,2
$$

(см. (1.6.1), (1.6.2)). Тогда эти условия порождают начальный элемент

$$
\tilde{z}^{0}=\left(\boldsymbol{u}_{1}^{0} ; \mathbf{0} ; \mathbf{0} ; \zeta^{0} ; \nabla \Phi_{21}^{0} ; p_{2}^{0}\right)^{\tau} \in \mathcal{D}(\tilde{B}) \subset \mathcal{D}(\overline{\tilde{B}}) .
$$

Аналогично проверяем, что если выполнено условие (1.7.2), то заданная функция $\tilde{f}(t)$ обладает свойством

$$
\tilde{f}(t) \in C^{1}([0, T] ; \tilde{\mathcal{H}})
$$

(см. (1.6.5)). Воспользуемся теперь тем фактом, что оператор $\tilde{C}$ в (1.7.3) положительно определен и ограничен в $\tilde{\mathcal{H}}$, и введем в $\tilde{\mathcal{H}}$ эквивалентную норму, порожденную скалярным произведением

$$
(\tilde{z}, \tilde{y})_{\tilde{C}}:=(\tilde{C} \tilde{z}, \tilde{y})_{\tilde{\mathcal{H}}} \cdot
$$

Перепишем затем задачу (1.7.3) в виде

$$
\frac{d \tilde{z}}{d t}=-\tilde{C}^{-1} \bar{B} \tilde{z}+\tilde{C}^{-1} \tilde{f}(t), \quad \tilde{z}(0)=\tilde{z}^{0}
$$

и заметим, что оператор $-\tilde{C}^{-1} \overline{\tilde{B}}$ является максимальным диссипативным в пространстве $\tilde{\mathcal{H}}_{\tilde{C}}$ со скалярным произведением (1.7.6). Поэтому (см., например, [12, с. 166]) при условиях (1.7.4), (1.7.5) задача (1.7.7) имеет единственное сильное решение $\tilde{z}(t)$, т.е. такое, что все слагаемые в $(1.7 .7)$ являются элементами из $C\left([0, T] ; \tilde{\mathcal{H}}_{\tilde{C}}\right)$. Отсюда следует, что $\tilde{z}(t)$ является также сильным решением задачи Коши (1.7.3), где все слагаемые являются непрерывными функциями $t$ со значениями в $\tilde{\mathcal{H}}$.

2. Выпишем теперь эту систему уравнений построчно с учетом представления операторной матрицы $\bar{B}$ в факторизованном виде (см. (1.6.7), (1.6.8), (1.6.13)-(1.6.15)). Имеем задачу

$$
\begin{gathered}
\frac{d}{d t}\left\{\left[\rho_{1} \boldsymbol{u}_{1}+\left(\rho_{2,0}(0)\right)^{2}\left(Q \gamma_{2}\right)\left(V \gamma_{n, 1}\right) \boldsymbol{u}_{1}\right]-\rho_{2,0}(0)\left(Q P_{\Gamma} \gamma_{2} \nabla^{-1}\right)\left(\nabla \Phi_{21}\right)\right\}+ \\
+\mu_{1}^{1 / 2} A_{1}^{1 / 2}\left\{\mu_{1}^{1 / 2} A_{1}^{1 / 2} \boldsymbol{u}_{1}+\sum_{k=1}^{2} \alpha_{k}^{1 / 2} \boldsymbol{w}_{k}+g \mu_{1}^{-1 / 2}\left(\rho_{1}-\rho_{2,0}(0)\right)\left(\gamma_{n, 1} A_{1}^{-1 / 2}\right)^{*} \zeta\right\}= \\
=\rho_{1} P_{0, S_{1}} \boldsymbol{f}_{1}-\rho_{2,0}(0) Q P_{\Gamma} \gamma_{2} F_{2}, \\
\nabla F_{2}=P_{G, 2} \boldsymbol{f}_{2}, \quad \boldsymbol{u}_{1}(0)=\boldsymbol{u}_{1}^{0}, \\
\frac{\boldsymbol{w}_{1}}{d t}-\mu_{1}^{1 / 2} \alpha_{1}^{1 / 2} A_{1}^{1 / 2} \boldsymbol{u}_{1}+\beta_{1} \boldsymbol{w}_{1}=\mathbf{0}, \quad \boldsymbol{w}_{1}(0)=\mathbf{0}, \\
\frac{\boldsymbol{w}_{2}}{d t}-\mu_{1}^{1 / 2} \alpha_{2}^{1 / 2} A_{1}^{1 / 2} \boldsymbol{u}_{1}+\beta_{1} \boldsymbol{w}_{2}=\mathbf{0}, \quad \boldsymbol{w}_{2}(0)=\mathbf{0}, \\
g\left(\rho_{1}-\rho_{2,0}(0)\right) \frac{d \zeta}{d t}-g\left(\rho_{1}-\rho_{2,0}(0)\right) \gamma_{n, 1} \boldsymbol{u}_{1}=0, \quad \zeta(0)=\zeta^{0} \\
\frac{d}{d t}\left(\nabla \Phi_{21}-\rho_{2,0}(0) \nabla\left(V \gamma_{n, 1} \boldsymbol{u}_{1}\right)\right)+\nabla\left(\rho_{2,0}(0)^{-1} p_{2}\right)=P_{G, 2} \boldsymbol{f}_{2}=: \nabla F_{2}, \quad \nabla \Phi_{21}(0)=\nabla \Phi_{21}^{0}, \\
a^{-2} \frac{d p_{2}}{d t}+\operatorname{div}\left(\rho_{2,0} \nabla \Phi_{21}\right)=0, \quad p_{2}(0)=p_{2} .
\end{gathered}
$$


Заметим, что система уравнений, отвечающая задаче (1.6.4) с незамкнутым оператором $\tilde{B}$, отличается от (1.7.8) лишь тем, что в первом уравнении (1.7.8а) слева раскрыты вторые скобки, а также оператор $\left(\gamma_{n, 1} A_{1}^{-1 / 2}\right)^{*}$ заменен на $A_{1}^{-1} Q$ (см. лемму 1.8). Таким образом, теперь возникает проблема доказать, что при условиях данной теоремы (см. (1.7.1), (1.7.2)) можно раскрыть упомянутые скобки, и тогда все полученные слагаемые в первом уравнении (1.7.8а) будут элементами из $C\left([0, T] ; \boldsymbol{J}_{0, S_{1}}\left(\Omega_{1}\right)\right)$.

3. Переходя к доказательству этого факта, отметим, что для сильного решения задачи (1.7.3) функция переменной $t$ в упомянутых скобках является элементом из $C\left([0, T] ; \mathcal{D}\left(A_{1}^{1 / 2}\right)\right)=$ $C\left([0, T] ; \boldsymbol{J}_{0, S_{1}}^{1}\left(\Omega_{1}\right)\right)$. Но тогда

$$
\begin{aligned}
\boldsymbol{v}_{1}:=\left\{\boldsymbol{u}_{1}+\sum_{k=1}^{2} \alpha_{k}^{1 / 2} \mu_{1}^{-1 / 2} A_{1}^{-1 / 2} \boldsymbol{w}_{k}+g \mu_{1}^{-1 / 2}\left(\rho_{1}-\rho_{2,0}(0)\right) A_{1}^{-1 / 2}\left(\gamma_{n, 1} A_{1}^{-1 / 2}\right)^{*} \zeta\right\} \in & \\
\in & \in C\left([0, T] ; \mathcal{D}\left(A_{1}\right)\right) .
\end{aligned}
$$

Однако из уравнений $(1.7 .8 \mathrm{~b}),(1.7 .8 \mathrm{c}),(1.7 .8 \mathrm{~d})$ задачи (1.7.8) следует (см. (1.6.1), (1.6.2)), что

$$
\boldsymbol{w}_{k}(t)=\mu_{1}^{1 / 2} \alpha_{k}^{1 / 2} A_{1}^{1 / 2} \int_{0}^{t} e^{-\beta_{k}(t-s)} \boldsymbol{u}_{1}(s) d s, \quad k=1,2, \quad \zeta(t)=\int_{0}^{t} \gamma_{n, 1} \boldsymbol{u}_{1}(s) d s+\zeta^{0} .
$$

Подставляя эти выражения в (1.7.9), приходим к соотношению

$$
\begin{aligned}
\boldsymbol{u}_{1}(t)+\sum_{k=1}^{2} \alpha_{k}^{1 / 2} \int_{0}^{t} e^{-\beta_{k}(t-s)} \boldsymbol{u}_{1}(s) d s+ & \\
& +g \mu_{1}^{-1}\left(\rho_{1}-\rho_{2,0}(0)\right) A_{1}^{-1 / 2}\left(\gamma_{n, 1} A_{1}^{-1 / 2}\right)^{*}\left(\int_{0}^{t} \gamma_{n, 1} \boldsymbol{u}_{1}(s) d s+\zeta^{0}\right)= \\
& =\boldsymbol{v}_{1}(t) \in C\left([0, T] ; \mathcal{D}\left(A_{1}\right)\right) .
\end{aligned}
$$

Рассмотрим (1.7.11) как интегральное уравнение Вольтерра в пространстве $\mathcal{H}\left(A_{1}\right)$ с нормой графика оператора $A_{1}$. Тогда в силу леммы 1.8 имеем для $\boldsymbol{u}_{1}(t) \in C\left([0, T] ; \mathcal{H}\left(A_{1}\right)\right)=$ $C\left([0, T] ; \mathcal{D}\left(A_{1}\right)\right)$ соотношение

$$
A_{1}^{-1 / 2}\left(\gamma_{n, 1} A_{1}^{-1 / 2}\right)^{*}\left(\gamma_{n, 1} \boldsymbol{u}_{1}\right)=A_{1}^{-1 / 2}\left(A_{1}^{-1 / 2} Q\right)\left(\gamma_{n, 1} \boldsymbol{u}_{1}\right)=A_{1}^{-1}\left(Q \gamma_{n, 1} \boldsymbol{u}_{1}\right) \in C\left([0, T] ; \mathcal{D}\left(A_{1}\right)\right)
$$

(см. лемму 1.2 и свойство (1.5.4)). Отсюда следует, что в интегральном уравнении Вольтерра (1.7.11) ядро является непрерывной по $t, s$ оператор функцией в треугольнике $\Delta_{T}:=\{(t, s)$ : $0 \leqslant s \leqslant t \leqslant T\}$, принимающей значения из $\mathcal{L}\left(\mathcal{H}\left(A_{1}\right)\right)$. Поэтому уравнение (1.7.11) имеет единственное решение $\boldsymbol{u}_{1}(t) \in C\left([0, T] ; \mathcal{D}\left(A_{1}\right)\right)$, причем все остальные слагаемые в (1.7.11) также принадлежат этому пространству.

4. Но тогда в уравнении (1.7.8a) можно раскрыть скобки во втором слагаемом слева, а затем из системы исключить функции $\boldsymbol{w}_{k}(t), k=1,2$ (см. (1.7.10)). В итоге возникает система уравнений (1.4.13)-(1.4.16), где все слагаемые являются непрерывными функциями $t$ со значениями в соответствующих пространствах. Значит, задача (1.4.13)-(1.4.16), т.е. задача (1.5.3), имеет единственное сильное решение в смысле определения 1.1.

В качестве замечания к теореме 1.1 отметим, что для сильного решения задачи (1.5.3) при условиях (1.7.1), (1.7.2) имеют место следующие свойства:

$$
\begin{gathered}
\boldsymbol{u}_{1} \in C\left([0, T] ; \mathcal{D}\left(A_{1}\right)\right) \cap C^{1}\left([0, T] ; \boldsymbol{J}_{0, S_{1}}\left(\Omega_{1}\right)\right) ; \\
\nabla \Phi_{21} \in C^{1}\left([0, T] ; \boldsymbol{G}\left(\Omega_{2} ; \rho_{2,0}\right)\right) ; \quad \zeta \in C^{1}\left([0, T] ; H_{\Gamma}^{1 / 2}\right) ; \\
p_{2} \in C^{1}\left([0, T] ; L_{2, \Omega_{2}, \rho_{2,0}^{-1}}\right) \cap C\left([0, T] ; H_{\Omega_{2}}^{1}\left(\Omega_{2} ; \rho_{2,0}\right)\right) .
\end{gathered}
$$


Опираясь на теорему 1.1, можно получить утверждение об однозначной разрешимости исходной начально-краевой задачи (1.1.5)-(1.1.13) на произвольном отрезке времени $[0, T]$.

\subsection{K проблеме нормальных колебаний системы «вязкоупругая жидкость-} баротропный газ». Рассмотрим решения однородной задачи (1.7.3), зависящие от времени по закону

$$
\tilde{z}(t)=\tilde{z} e^{-\lambda t} .
$$

Функции такого вида называют нормальными движениями исследуемой системы, числа $\lambda$ - комплексными декрементами затухания нормальных движений, а $\tilde{z} \neq 0$-амплитудными элементами.

Для нахождения амплитудных элементов и чисел $\lambda$ возникает из (1.7.3) спектральная задача

$$
\overline{\tilde{B}} \tilde{z}=\lambda \tilde{C} \tilde{z}, \quad \tilde{z} \in \mathcal{D}(\overline{\tilde{B}}) \subset \tilde{\mathcal{H}},
$$

которая, в частности, получается из системы уравнений (1.7.8) и выглядит следующим образом:

$$
\begin{gathered}
\mu_{1}^{1 / 2} A_{1}^{1 / 2}\left\{\mu_{1}^{1 / 2} A_{1}^{1 / 2} \boldsymbol{u}_{1}+\alpha_{1}^{1 / 2} \boldsymbol{w}_{1}+\alpha_{2}^{1 / 2} \boldsymbol{w}_{2}+g \mu_{1}^{-1 / 2}\left(\rho_{1}-\rho_{2,0}(0)\right)\left(\gamma_{n, 1} A_{1}^{-1 / 2}\right)^{*} \zeta\right\}= \\
\left.=\lambda\left[\rho_{1} \boldsymbol{u}_{1}+\left(\rho_{2,0}(0)\right)^{2}\left(Q \gamma_{2}\right)\left(V \gamma_{n, 1}\right) \boldsymbol{u}_{1}\right]-\rho_{2,0}(0) Q P_{\Gamma} \gamma_{2} \Phi_{21}\right), \\
-\mu_{1}^{1 / 2} \alpha_{1}^{1 / 2} A_{1}^{1 / 2} \boldsymbol{u}_{1}+\beta_{1} \boldsymbol{w}_{1}=\lambda \boldsymbol{w}_{1} \\
-\mu_{1}^{1 / 2} \alpha_{2}^{1 / 2} A_{1}^{1 / 2} \boldsymbol{u}_{1}+\beta_{2} \boldsymbol{w}_{2}=\lambda \boldsymbol{w}_{2} \\
-g\left(\rho_{1}-\rho_{2,0}(0)\right) \gamma_{n, 1} \boldsymbol{u}_{1}=\lambda g\left(\rho_{1}-\rho_{2,0}(0)\right) \zeta \\
\nabla\left(\rho_{2,0}(0)^{-1} p_{2}\right)=\lambda\left(\nabla \Phi_{21}-\rho_{2,0}(0) \nabla\left(V \gamma_{n, 1} \boldsymbol{u}_{1}\right)\right) \\
\operatorname{div}\left(\rho_{2,0} \nabla \Phi_{21}\right)=\lambda a^{-2} p_{2} .
\end{gathered}
$$

Наша дальнейшая цель - исключить из системы уравнений (1.8.1) часть неизвестных, перейти к спектральной задаче для операторного пучка (оператор-функции от $\lambda$ ) с ограниченными операторными коэффициентами и исследовать ее методами спектральной теории (см., например, [15]).

Установим сначала следующий простой факт.

Лемма 1.15. Числа $\lambda=0, \lambda=\beta_{k}, k=1,2$, не являются собственными значениями спектральной задачи (1.8.1).

Доказательство. 1. Положим $\lambda=0$ в (1.8.1). Тогда из предпоследнего уравнения (1.8.1e) получаем, что $\nabla\left(\rho_{2,0}(0)^{-1} p_{2}\right)=\mathbf{0}$. Так как это элемент из $\boldsymbol{G}\left(\Omega_{2} ; \rho_{2,0}\right)($ см. $(1.2 .9))$, то $p_{2}=0$. Далее, из последнего уравнения (1.8.1f) и граничных условий для $\Phi_{21}$ возникает задача

$$
-\Delta_{0} \Phi_{21}=0 \quad \text { в } \Omega_{2}, \quad \rho_{2,0} \frac{\partial \Phi_{21}}{\partial n}=0 \quad \text { на } \partial \Omega_{2}, \quad \int_{\Omega_{2}} \rho_{2,0} \Phi_{21} d \Omega_{2}=0,
$$

которая имеет лишь тривиальное решение: $\Phi_{21}=0$.

Из уравнений $(1.8 .1 \mathrm{~b})-(1.8 .1 \mathrm{~d})$ имеем также связи

$$
\beta_{k} \boldsymbol{w}_{k}=\mu_{1}^{1 / 2} \alpha_{k} \beta_{k}^{-1} A_{1}^{1 / 2} \boldsymbol{u}_{1}, \quad k=1,2, \quad \gamma_{n, 1} \boldsymbol{u}_{1}=0,
$$

а тогда из первого уравнения (1.8.1а) получаем соотношение

$$
\mu_{1}^{1 / 2}\left(1+\sum_{k=1}^{2} \frac{\alpha_{k}}{\beta_{k}}\right) A_{1}^{1 / 2} \boldsymbol{u}_{1}+g \mu_{1}^{-1 / 2}\left(\rho_{1}-\rho_{2,0}(0)\right)\left(\gamma_{n, 1} A_{1}^{-1 / 2}\right)^{*} \zeta=\mathbf{0} .
$$

Отсюда следует, что

$$
\left(1+\sum_{k=1}^{2} \frac{\alpha_{k}}{\beta_{k}}\right)\left\|\mu_{1}^{1 / 2} A_{1}^{1 / 2} \boldsymbol{u}_{1}\right\|_{\boldsymbol{L}_{2}\left(\Omega_{1}\right)}^{2}=0
$$


так как

$$
\left(\left(\gamma_{n, 1} A_{1}^{-1 / 2}\right)^{*} \zeta, A_{1}^{1 / 2} \boldsymbol{u}_{1}\right)_{\boldsymbol{L}_{2}\left(\Omega_{1}\right)}=\left(\zeta,\left(\gamma_{n, 1} A_{1}^{-1 / 2}\right) A_{1}^{1 / 2} \boldsymbol{u}_{1}\right)_{L_{2, \Gamma}}=\left(\zeta, \gamma_{n, 1} \boldsymbol{u}_{1}\right)_{L_{2, \Gamma}}=0
$$

Значит, $\boldsymbol{u}_{1}=\mathbf{0}$.

Наконец, из оставшейся связи $\left(\gamma_{n, 1} A_{1}^{-1 / 2}\right)^{*} \zeta=0$ при любом $\boldsymbol{\eta} \in \boldsymbol{J}_{0, S_{1}}\left(\Omega_{1}\right)$ имеем

$$
\left(\boldsymbol{\eta},\left(\gamma_{n, 1} A_{1}^{-1 / 2}\right)^{*} \zeta\right)_{\boldsymbol{J}_{0, S_{1}}\left(\Omega_{1}\right)}=\left(\gamma_{n, 1} A_{1}^{-1 / 2} \boldsymbol{\eta}, \zeta\right)_{L_{2, \Gamma}}=0
$$

и так как совокупность элементов вида $\gamma_{n, 1} A_{1}^{-1 / 2} \eta$ при $\boldsymbol{\eta} \in \boldsymbol{J}_{0, S_{1}}\left(\Omega_{1}\right)$ образует множество $H_{\Gamma}^{1 / 2}$, плотное в $L_{2, \Gamma}$, то $\zeta=0$. Это доказывает первое утверждение леммы.

2. Положим теперь $\lambda=\beta_{1}$ в (1.8.1). Тогда из второго уравнения (1.8.1b) получаем $A_{1}^{1 / 2} \boldsymbol{u}_{1}=\mathbf{0}$ и потому $\boldsymbol{u}_{1}=\mathbf{0}$. Поэтому из третьего уравнения $(1.8 .1 \mathrm{c})$ следует $\left(\beta_{1} \neq \beta_{2}\right)$, что $\boldsymbol{w}_{2}=\mathbf{0}$, а из четвертого (1.8.1d) имеем $\zeta=0$. Далее, для функций $\Phi_{21}$ и $p_{2}$ возникает задача

$$
\begin{gathered}
\nabla\left(\rho_{2,0}(0)^{-1} p_{2}\right)=\beta_{1} \nabla \Phi_{21}, \quad \operatorname{div}\left(\rho_{2,0} \nabla \Phi_{21}\right)=\beta_{1} a^{-2} p_{2} \quad \text { в } \Omega_{2}, \\
\rho_{2,0} \frac{\partial \Phi_{21}}{\partial n}=0 \quad \text { на } \partial \Omega_{2}, \quad \int_{\Omega_{2}} \rho_{2,0} \Phi_{21} d \Omega_{2}
\end{gathered}
$$

которая приводится к задаче на собственные значения

$$
A_{2} \Phi_{21}=-\beta_{1}^{2} \Phi_{21}, \quad \Phi_{21} \in H_{\Omega_{2}}^{1}\left(\Omega_{2} ; \rho_{2,0}\right)
$$

(см. (1.3.4), (1.3.5), (1.3.12)). Поскольку оператор $A_{2}$ имеет дискретный положительный спектр, то эта задача имеет лишь нулевое решение, и потому $\nabla \Phi_{21}=0, p_{2}=0$. Теперь из первого уравнения (1.8.1а) получаем, что $\alpha_{1}^{1 / 2} \boldsymbol{w}_{1}=\mathbf{0}$, т.е. $\boldsymbol{w}_{1}=\mathbf{0}$.

Случай $\lambda=\beta_{2}$ рассматривается аналогично, что и завершает доказательство.

Опираясь на лемму 1.15 , исключим при $\lambda \neq 0, \lambda \neq \beta_{k}, k=1,2$, в уравнениях (1.8.1) все искомые элементы, кроме $\boldsymbol{u}_{1}$ и $\nabla \Phi_{21}$. Из соотношений $(1.8 .1 \mathrm{~b})-(1.8 .1 \mathrm{~d})$ имеем

$$
\boldsymbol{w}_{k}=\frac{\mu_{1}^{1 / 2} \alpha_{k}^{1 / 2}}{\beta_{k}-\lambda} A_{1}^{1 / 2} \boldsymbol{u}_{1}, \quad k=1,2, \quad \zeta=-\lambda^{-1} \gamma_{n, 1} \boldsymbol{u}_{1}
$$

Подставляя их в первое уравнение (1.8.1а) и осуществляя замену

$$
A_{1}^{1 / 2} \boldsymbol{u}_{1}=\boldsymbol{\eta}_{1} \in \boldsymbol{J}_{0, S_{1}}\left(\Omega_{1}\right)
$$

приходим к уравнению

$$
\begin{array}{r}
\mu_{1} e_{2}(\lambda) \boldsymbol{\eta}_{1}=\lambda\left\{A_{1}^{-1 / 2}\left(\rho_{1} I_{1}+\left(\rho_{2,0}(0)\right)^{2}\left(Q \gamma_{2}\right)\left(V \gamma_{n, 1}\right)\right) A_{1}^{-1 / 2} \boldsymbol{\eta}_{1}-\rho_{2,0}(0) A_{1}^{-1 / 2} Q P_{\Gamma} \gamma_{2} \Phi_{21}\right\}+ \\
+\lambda^{-1} g\left(\rho_{1}-\rho_{2,0}(0)\right)\left(\gamma_{n, 1} A_{1}^{-1 / 2}\right)^{*}\left(\gamma_{n, 1} A_{1}^{-1 / 2}\right) \boldsymbol{\eta}_{1}
\end{array}
$$

где

$$
e_{2}(\lambda):=1+\sum_{k=1}^{2} \frac{\alpha_{k}}{\beta_{k}-\lambda}
$$

Уравнения (1.8.1е) и (1.8.1f) приводят к связи

$$
A_{2} \Phi_{21}=-\lambda^{2} a^{-2}\left(\Phi_{21}-\rho_{2,0} V\left(\gamma_{n, 1} A_{1}^{-1 / 2}\right) \eta_{1}\right) .
$$

Осуществляя еще здесь замену

$$
A_{2}^{1 / 2} \Phi_{21}=\varphi_{2} \in L_{2, \Omega_{2}, \rho_{2,0}}
$$

(см. (1.3.12), (1.3.13)), получим окончательно из (1.8.2)-(1.8.4) задачу на собственные значения

$$
\mu_{1} e_{2}(\lambda) \boldsymbol{\eta}_{1}=\lambda\left(A_{11} \boldsymbol{\eta}_{1}+A_{12} \varphi_{2}\right)+\lambda^{-1} B_{1} \boldsymbol{\eta}_{1}, \quad \varphi_{2}=-\lambda^{2} a^{-2}\left(A_{21} \boldsymbol{\eta}_{1}+A_{22} \varphi_{2}\right),
$$


где

$$
\begin{gathered}
A_{11}:=A_{1}^{-1 / 2}\left(\rho_{1} I_{1}+\left(\rho_{2,0}(0)\right)^{2}\left(Q \gamma_{2}\right)\left(V \gamma_{n, 1}\right)\right) A_{1}^{-1 / 2}, \quad A_{22}:=A_{2}^{-1}, \\
A_{12}:=-\rho_{2,0}(0) A_{1}^{-1 / 2} Q P_{\Gamma} \gamma_{2} A_{1}^{-1 / 2}, \quad A_{21}:=-\rho_{2,0}(0)\left(A_{2}^{-1 / 2} V\right)\left(\gamma_{n, 1} A_{1}^{-1 / 2}\right), \\
B_{1}:=g\left(\rho_{1}-\rho_{2,0}(0)\right)\left(\gamma_{n, 1} A_{1}^{-1 / 2}\right)^{*}\left(\gamma_{n, 1} A_{1}^{-1 / 2}\right) .
\end{gathered}
$$

Перепишем задачу (1.8.5)-(1.8.6) в векторно-матричном виде в двух формах. Первая форма такова:

$$
\left(\begin{array}{cc}
\mu_{1} e_{2}(\lambda) I_{1} & 0 \\
0 & 0
\end{array}\right)\left(\begin{array}{l}
\boldsymbol{\eta}_{1} \\
\varphi_{2}
\end{array}\right)=\lambda\left(\begin{array}{ll}
A_{11} & A_{12} \\
A_{21} & A_{22}
\end{array}\right)\left(\begin{array}{l}
\boldsymbol{\eta}_{1} \\
\varphi_{2}
\end{array}\right)+\lambda^{-1}\left(\begin{array}{cc}
B_{1} & 0 \\
0 & a^{2} I_{2}
\end{array}\right)\left(\begin{array}{l}
\boldsymbol{\eta}_{1} \\
\varphi_{2}
\end{array}\right)
$$

Вторая форма выглядит следующим образом:

$$
\begin{aligned}
\left(\begin{array}{cc}
\mu_{1} e_{2}(\lambda) I_{1} & 0 \\
0 & I_{2}
\end{array}\right)\left(\begin{array}{l}
\boldsymbol{\eta}_{1} \\
\varphi_{2}
\end{array}\right)=\lambda\left(\begin{array}{cc}
A_{11} & A_{12} \\
0 & 0
\end{array}\right) & \left(\begin{array}{l}
\boldsymbol{\eta}_{1} \\
\varphi_{2}
\end{array}\right)- \\
& -\lambda^{2} a^{-2}\left(\begin{array}{cc}
0 & 0 \\
A_{21} & A_{22}
\end{array}\right)\left(\begin{array}{l}
\boldsymbol{\eta}_{1} \\
\varphi_{2}
\end{array}\right)+\lambda^{-1}\left(\begin{array}{cc}
B_{1} & 0 \\
0 & 0
\end{array}\right)\left(\begin{array}{l}
\boldsymbol{\eta}_{1} \\
\varphi_{2}
\end{array}\right)
\end{aligned}
$$

где $\left(\boldsymbol{\eta}_{1} ; \varphi_{2}\right)^{\tau} \in \boldsymbol{J}_{0, S_{1}}\left(\Omega_{1}\right) \oplus L_{2, \Omega_{2}, \rho_{2,0}}$.

Лемма 1.16. Операторные коэфбициенты $A_{j k}, j, k=1,2$, компактнъ, причем $A_{j k}=A_{k j}^{*}$, $A_{k k} \geqslant 0$. Кроме того, операторный коэффичиент $B_{1}$ - компактный неотрицательный оператор, действующий в $\boldsymbol{J}_{0, S_{1}}\left(\Omega_{1}\right)$.

Доказательство. Эти свойства следуют непосредственно из определений (1.8.6) операторов и лемм 2.3, 1.2-1.4, 1.8, 1.13, а также свойств, описанных в (1.3.13), (1.3.17), (1.4.10).

Опираясь на эти факты, укажем на некоторые простые свойства спектра исследуемой задачи (1.8.5):

I. Спектр задачи (1.8.5) симметричен относительно вещественной оси.

II. Спектр задачи (1.8.5) дискретен, т.е. состоит из счетного множества конечнократных собственных значений с возможными предельным точками $\lambda=0, \lambda=\infty, \lambda=\beta_{k}, k=1,2$, а также точками $\lambda=\delta_{k}, e_{2}\left(\delta_{k}\right)=0, k=1,2$, т.е. нулями функции $e_{2}(\lambda)$.

III. Спектр задачи (1.8.5)-(1.8.6) расположен в правой комплексной полуплоскости.

Доказательство. І. Перепишем задачу (1.8.7) в кратком виде

$$
L_{1}(\lambda) \xi=0, \quad \xi:=\left(\boldsymbol{\eta}_{1} ; \varphi_{2}\right)^{\tau}
$$

и заметим, что операторный пучок $L_{1}(\lambda)$ самосопряжен (см. [15]), т.е. $\left(L_{1}(\lambda)\right)^{*}=L_{1}(\lambda)$. Отсюда и следует данное утверждение.

II. Задачу (1.8.8) перепишем в виде

$$
L_{2}(\lambda) \xi=0, \quad \xi=\left(\boldsymbol{\eta}_{1} ; \varphi_{2}\right)^{\tau}
$$

и заметим, что она приводится к спектральной проблеме

$$
(I+\Phi(\lambda)) \xi=0
$$

где $I$ - единичный оператор в $\boldsymbol{J}_{0, S_{1}}\left(\Omega_{1}\right) \oplus L_{2, \Omega_{2}, \rho_{2,0}}$, а $\Phi(\lambda)$ - аналитическая оператор-функция, принимающая компактные значения. Заметим теперь, что оператор $\left.(I+\Phi(\lambda))\right|_{\lambda=-1}$ обратим, так каK

$$
L_{1}(-1)=\left(\begin{array}{cc}
\mu_{1} e_{2}(-1) I_{1} & 0 \\
0 & a^{-2} I_{2}
\end{array}\right)+\left(\begin{array}{cc}
A_{11} & A_{12} \\
A_{21} & A_{22}
\end{array}\right)+a^{-2}\left(\begin{array}{cc}
B_{1} & 0 \\
0 & 0
\end{array}\right)
$$

- положительно определенный оператор, поскольку операторная матрица $\left(A_{j k}\right)_{j k=1}^{2}$-компактный положительный оператор (факт положительности проверяется непосредственно), а $e_{2}(-1)>0$. Поэтому по теореме Келдыша (см. [7]; иногда ее называют теоремой Гохберга, см. [6]) получаем доказываемое утверждение.

III. Доказательство основано на неравенстве 


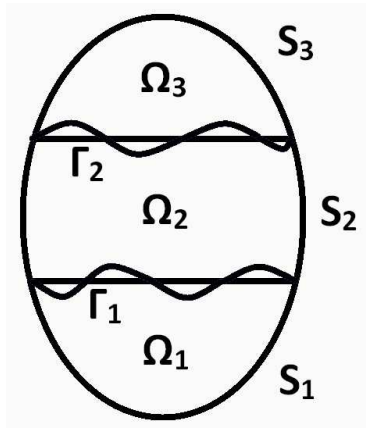

Рис. 2

$$
\begin{aligned}
\operatorname{Re} \lambda=\left\{\mu_{1}\left(1+\sum_{k=1}^{2} \frac{\alpha_{k} \beta_{k}}{\left|\beta_{k}-\lambda\right|^{2}}\right)\left\|\boldsymbol{\eta}_{1}\right\|_{\boldsymbol{L}_{2}\left(\Omega_{1}\right)}^{2}+\|\varphi\|_{L_{2, \Omega_{2}, \rho_{2}, 0}}^{2}\right\} / \\
/\left\{\sum_{k=1}^{2} \frac{\alpha_{k}}{\left|\beta_{k}-\lambda\right|^{2}}\left\|\boldsymbol{\eta}_{1}\right\|_{\boldsymbol{L}_{2}\left(\Omega_{1}\right)}^{2}+(A \xi, \xi)+|\lambda|^{-2}\left(B_{a} \xi, \xi\right)\right\}>0,
\end{aligned}
$$

которое можно вывести из уравнения (1.8.7) либо (1.8.9), и том факте, что квадратичные формы операторных матриц $A$ и $B_{a}$ в правой части (1.8.7) являются соответственно положительной и неотрицательной.

Дальнейшее подробное исследование свойств решений задачи (1.8.5)-(1.8.6) будет проведено в другой работе. Будут получены свойства полноты и базисности системы корневых (собственных и присоединенных) элементов этой задачи, наличие шести ветвей собственных значений, их асимптотическое поведение и физический смысл. Будут рассмотрены также и другие близкие вопросы.

\section{2. О МАЛЫХ ДВИЖЕНИЯХ ГИДРОСИСТЕМЫ ИЗ ТРЕХ НЕСМЕШИВАЮЩИХСЯ ЖИДКОСТЕЙ, ЗАПОЛНЯЮЩИХ НЕПОДВИЖНЫЙ СОСУД}

2.1. Постановка задачи. Закон баланса полной энергии системы. Рассмотрим неподвижный сосуд $\Omega$, полностью заполненный системой из трех несмешивающихся жидкостей. Жидкости предполагаются тяжелыми, и в силу этого действие капиллярных сил в этой задаче не учитывается. Область $\Omega_{1}$, нижняя по отношению к действию силы тяжести, заполнена несжимаемой вязкоупругой жидкостью обобщенной модели Олдройта (см., например, $[17,18,20,23])$. Далее, как и выше, $\rho_{1}, \mu_{1}$ - соответственно плотность и динамический коэффициент вязкости вязкоупругой жидкости. Области $\Omega_{2}$ и $\Omega_{3}$ заполнены идеальными несжимаемыми жидкостями с плотностями $\rho_{2}$ и $\rho_{3}$.

Обозначим через $\boldsymbol{n}_{k}$ единичный вектор, нормальный к $\partial \Omega_{k}$ и направленный вне $\Omega_{k}, k=\overline{1,3}$. Через $S_{k}$ обозначим часть стенки сосуда, граничащей с областью $\Omega_{k}, k=\overline{1,3}$. Горизонтальную границу раздела между вязкоупругой и идеальной жидкостями в состоянии равновесия обозначим через $\Gamma_{1}$, а между идеальными жидкостями - через $\Gamma_{2}$. Введем систему координат $O x_{1} x_{2} x_{3}$, жестко связанную с сосудом, таким образом, чтобы ось $O x_{3}$ была направлена против действия силы тяжести. Тогда ускорение гравитационного поля $\boldsymbol{g}=-g \boldsymbol{e}_{3}, g>0$, а в состоянии покоя поля давлений в жидкостях выражаются по законам

$$
P_{0, k}\left(x_{3}\right)=c_{k}-\rho_{k} g x_{3}, \quad k=\overline{1,3},
$$

где константы $c_{k}$ определяются из условия равенства давлений на границах раздела $\Gamma_{j}, j=1,2$, при $x_{3}=0$.

Теперь перейдем к уравнениям, описывающим движение гидросистемы. Обозначим через $\boldsymbol{u}_{k}$ поля скоростей жидкостей в $\Omega_{k}, k=\overline{1,3}$, а через $p_{k}(t, x)$ - отклонения полей давлений от их равновесных значений (см. (2.1.1)). Кроме того, полагаем, что на исследуемую гидродинамическую 
систему дополнительно к гравитационному полю действует малое поле внешних сил $\boldsymbol{f}=\boldsymbol{f}(t, x)$, $x \in \Omega$.

Тогда линеаризованные уравнения движения жидкостей имеют следующий вид (см., например, $[22,23]):$

$$
\begin{gathered}
\rho_{1} \frac{\partial \boldsymbol{u}_{1}}{\partial t}=-\nabla p_{1}+\mu_{1} \Delta \boldsymbol{v}_{1}+\rho_{1} \boldsymbol{f}_{1}(t, x), \quad \operatorname{div} \boldsymbol{u}_{1}=0 \quad \text { в } \Omega_{1}, \\
\boldsymbol{v}_{1}(t, x)=\boldsymbol{u}_{1}(t, x)+\sum_{r=1}^{m} \alpha_{r} \int_{0}^{t} e^{-\beta_{r}(t-s)} \boldsymbol{u}_{1}(s, x) d s=: I_{0,1}(t) \boldsymbol{u}_{1}, \\
\rho_{l} \frac{\partial \boldsymbol{u}_{l}}{\partial t}=-\nabla p_{l}+\rho_{l} \boldsymbol{f}_{l}(t, x), \quad \operatorname{div} \boldsymbol{u}_{l}=0 \quad \text { в } \Omega_{l}, \quad l=2,3,
\end{gathered}
$$

где $\alpha_{r} \geqslant 0, \beta_{r}>0, r=\overline{1, m},-$ коэффициенты, характеризующие свойства вязкоупругости жидкости обобщенной модели Олдройта, $\boldsymbol{f}_{k}(t, x):=\left.\boldsymbol{f}(t, x)\right|_{x \in \Omega_{k}}, k=\overline{1,3}$, а $\Delta$ - трехмерный оператор Лапласа.

Для вязкоупругой жидкости, как известно, на твердой стенке $S_{1}$ сосуда должно выполняться условие прилипания, т.е.

$$
\boldsymbol{u}_{1}=\mathbf{0} \quad \text { на } S_{1},
$$

а для идеальных на поверхностях $S_{l}, l=2,3,-$ условия непротекания

$$
\boldsymbol{u}_{l} \cdot \boldsymbol{n}_{l}=0 \quad \text { на } S_{l}, \quad l=2,3 .
$$

Будем описывать малые перемещения границ раздела между жидкостями с помощью функций вертикального отклонения

$$
x_{3}-h_{j}=: \zeta_{j}\left(t, x_{1}, x_{2}\right), \quad\left(x_{1}, x_{2}\right) \in \Gamma_{j}, \quad j=1,2,
$$

где $h_{j}$ - уровень равновесной поверхности $\Gamma_{j}, j=1,2$. Тогда на $\Gamma_{j}, j=1,2$, должны выполняться кинематические условия

$$
\frac{\partial \zeta_{j}}{\partial t}=\boldsymbol{u}_{j} \cdot \boldsymbol{n}_{j}=: \gamma_{n, j} \boldsymbol{u}_{j}=-\boldsymbol{u}_{j+1} \cdot \boldsymbol{n}_{j+1}=:-\gamma_{n, j+1} \boldsymbol{u}_{j+1}, \quad \boldsymbol{n}_{j}=\boldsymbol{e}_{3}=-\boldsymbol{n}_{j+1}, \quad j=1,2,
$$

а символом $\gamma_{n, j}, j=1,2$, обозначена операция взятия нормального следа на $\Gamma_{j}$, т.е. следа нормальной компоненты поля скорости. Заметим еще, что из условия сохранения объема каждой из жидкостей имеем интегральные связи

$$
\int_{\Gamma_{j}} \zeta_{j} d \Gamma_{j}=0, \quad j=1,2 .
$$

Сформулируем теперь динамические условия на $\Gamma_{j}, j=1,2$. Они состоят в том, что на движущейся границе раздела векторное поле напряжений при переходе от одной жидкости к другой изменяется непрерывно. Линеаризация этого условия и его снос на $\Gamma_{j}$ приводят к следующим соотношениям: на $\Gamma_{j}$ касательные напряжения (т.е. вдоль $\Gamma_{j}$ ) изменяются непрерывно, а нормальные напряжения (т.е. вдоль оси $O x_{3}$ ) компенсируются гравитационным скачком давлений. Имеем

$$
\begin{gathered}
\mu_{1} \tau_{q 3}\left(\boldsymbol{v}_{1}\right)=0, \quad q=1,2 ; \\
{\left[-p_{1}+\mu_{1} \tau_{33}\left(\boldsymbol{v}_{1}\right)\right]-\left[-p_{2}\right]=-g\left(\rho_{1}-\rho_{2}\right) \zeta_{1} \quad \text { на } \Gamma_{1},} \\
p_{2}-p_{3}=g\left(\rho_{2}-\rho_{3}\right) \zeta_{2} \quad \text { на } \Gamma_{2} .
\end{gathered}
$$

Здесь

$$
\tau_{q r}(\boldsymbol{u}):=\frac{\partial u_{q}}{\partial x_{r}}+\frac{\partial u_{r}}{\partial x_{q}}, \quad q, r=1,2,3,
$$

- удвоенный тензор скоростей деформаций. 
Наконец, для искомых функций $\boldsymbol{u}_{k}(t, x), k=\overline{1,3}$, и $\zeta_{j}\left(t, x_{1}, x_{2}\right), j=1,2$, необходимо еще задать начальные условия:

$$
\begin{gathered}
\boldsymbol{u}_{k}(0, x)=\boldsymbol{u}_{k}^{0}(x), \quad x \in \Omega_{k}, \quad \boldsymbol{u}_{j}^{0} \cdot \boldsymbol{n}_{j} \equiv-\boldsymbol{u}_{j+1}^{0} \cdot \boldsymbol{n}_{j+1}, \quad x \in \Gamma_{j}, \quad k=\overline{1,3}, \quad j=1,2, \\
\zeta_{j}(0, x)=\zeta_{j}^{0}(x), \quad x \in \Gamma_{j}, \quad j=1,2 .
\end{gathered}
$$

Закон баланса полной энергии. Будем считать, что задача (2.1.2)-(2.1.11) имеет классической решение, и выведем закон баланса полной энергии гидросистемы. Предварительно выпишем формулы Грина для векторных полей скоростей в областях $\Omega_{k}, k=\overline{1,3}$. Для дважды непрерывно дифференцируемых полей они имеют следующий вид:

$$
\begin{gathered}
\left.\mu_{1} E_{1}\left(\boldsymbol{\eta}_{1}, \boldsymbol{u}_{1}\right):=\frac{1}{2} \mu_{1} \int_{\Omega_{1}} \int_{q, r=1} \sum_{\Omega_{1}}^{3} \tau_{q r}\left(\boldsymbol{\eta}_{1}\right) \overline{\tau_{q r}\left(\boldsymbol{u}_{1}\right)}\right) d \Omega_{1}= \\
=\int_{\Omega_{1}} \boldsymbol{\eta}_{1} \cdot \overline{\left(-\mu_{1} \Delta \boldsymbol{u}_{1}+\nabla p_{1}\right)} d \Omega_{1}+\int \sum_{\Gamma_{1}}^{3} \eta_{1, q} \overline{\left(\mu_{1} \tau_{q, 3}\left(\boldsymbol{u}_{1}\right)-p_{1} \delta_{q 3}\right)} d \Gamma_{1}, \\
\operatorname{div} \boldsymbol{\eta}_{1}=\operatorname{div} \boldsymbol{u}_{1}=0 \quad \text { в } \Omega_{1}, \quad \boldsymbol{\eta}_{1}=\boldsymbol{u}_{1} \equiv \mathbf{0} \quad \text { на } S_{1}, \quad \boldsymbol{\eta}_{1}=\sum_{q=1}^{3} \eta_{1, q} \boldsymbol{e}_{q}, \\
\int_{\Omega_{2}} \boldsymbol{\eta}_{2} \cdot \overline{\nabla p_{2}} d \Omega_{2}=-\int_{\Gamma_{1}} \eta_{2,3} \overline{p_{2}} d \Gamma_{1}+\int_{\Gamma_{2}} \eta_{2,3} \overline{p_{2}} d \Gamma_{2}, \quad \int_{\Omega_{3}} \boldsymbol{\eta}_{3} \cdot \overline{\nabla p_{3}} d \Omega_{3}=-\int_{\Gamma_{2}} \eta_{3,3} \overline{p_{3}} d \Gamma_{2}, \\
\operatorname{div} \boldsymbol{\eta}_{l}=\operatorname{div} \boldsymbol{u}_{l}=0 \quad \text { в } \Omega_{l}, \quad \boldsymbol{\eta}_{l} \cdot \boldsymbol{\eta}_{l}=\boldsymbol{u}_{l} \cdot \boldsymbol{\eta}_{l} \equiv \mathbf{0} \quad \text { на } S_{l}, \quad l=2,3 .
\end{gathered}
$$

В этих формулах учтено, что направление внешних нормалей на $\Gamma_{j}, j=1,2$, для областей $\Omega_{k}$, $k=\overline{1,3}$, будет $\boldsymbol{n}_{j}=\boldsymbol{e}_{3}=-\boldsymbol{n}_{j+1}, j=1,2$.

Умножим обе части (2.1.2) и (2.1.4) слева на $\boldsymbol{u}_{k}, k=\overline{1,3}$, проинтегрируем по $\Omega_{k}$ соответственно и сложим результаты; будем иметь (для вещественнозначных полей):

$$
\sum_{k=1}^{3} \rho_{k} \int_{\Omega_{k}} \boldsymbol{u}_{k} \cdot \frac{\partial \boldsymbol{u}_{k}}{\partial t} d \Omega_{k}=-\sum_{k=1}^{3} \int_{\Omega_{k}} \boldsymbol{u}_{k} \cdot \nabla p_{k} d \Omega_{k}+\mu_{1} \int_{\Omega_{1}} \boldsymbol{u}_{1} \cdot\left(\Delta \boldsymbol{v}_{1}\right) d \Omega_{1}+\sum_{k=1}^{3} \rho_{k} \int_{\Omega_{k}} \boldsymbol{u}_{k} \cdot \boldsymbol{f}_{k} d \Omega_{k} .
$$

Используя формулы Грина (2.1.12), (2.1.13), а также граничные условия задачи (2.1.2)-(2.1.11), отсюда получаем соотношение

$$
\begin{aligned}
\frac{1}{2} \frac{d}{d t}\left\{\sum_{k=1}^{3} \rho_{k} \int_{\Omega_{k}}\left|\boldsymbol{u}_{k}\right|^{2} d \Omega_{k}\right\}= & -\mu_{1} E_{1}\left(\boldsymbol{u}_{1}, \boldsymbol{v}_{1}\right)+\sum_{k=1}^{3} \rho_{k} \int_{\Omega_{k}} \boldsymbol{u}_{k} \cdot \boldsymbol{f}_{k} d \Omega_{k}+ \\
& \left.+\int_{\Gamma_{1}} \sum_{q=1}^{3} u_{1, q}\left(\mu_{1} \tau_{q 3}\left(\boldsymbol{u}_{1}\right)-\left(p_{1}-p_{2}\right) \delta_{q 3}\right) d \Gamma_{1}+\int_{\Gamma_{2}} u_{2,3}\left(p_{3}-p_{2}\right)\right) d \Gamma_{2} .
\end{aligned}
$$

Учитывая еще соотношения (2.1.9) и (2.1.10), окончательно приходим к выводу, что

$$
\begin{aligned}
\frac{1}{2} \frac{d}{d t}\left\{\sum_{k=1}^{3} \rho_{k} \int_{\Omega_{k}}\left|\boldsymbol{u}_{k}\right|^{2} d \Omega_{k}+g\left(\rho_{1}-\rho_{2}\right) \int_{\Gamma_{1}}\left|\zeta_{1}\right|^{2} d \Gamma_{1}\right. & \left.+g\left(\rho_{2}-\rho_{3}\right) \int_{\Gamma_{2}}\left|\zeta_{2}\right|^{2} d \Gamma_{2}\right\}= \\
& =-\mu_{1} E_{1}\left(\boldsymbol{u}_{1}, \boldsymbol{v}_{1}\right)+\sum_{k=1}^{3} \rho_{k} \int_{\Omega_{k}} \boldsymbol{u}_{k} \cdot \boldsymbol{f}_{k} d \Omega_{k} .
\end{aligned}
$$


Это тождество есть закон баланса полной энергии системы в дифференциальной форме. Здесь в фигурных скобках стоит удвоенная полная (кинетическая плюс потенциальная) энергия гидросистемы, а справа - мощность диссипативных вязкоупругих сил и мощность дополнительных внешних сил, действующих на систему. После интегрирования (2.1.14) по $t$ в пределах от 0 до $t$ получаем закон баланса полной энергии в интегральной форме, т.е. на произвольном отрезке времени $(0, t)$.

2.2. Метод ортогонального проектирования. Переход к системе операторных уравнений. Опираясь на (2.1.14), выберем функциональные пространства, адекватные задаче, и проведем ее исследование по схеме, уже использованной в разделе 1.

Проектирование уравнений Эйлера. Воспользуемся далее разложением пространства векторных полей $\boldsymbol{L}_{2}\left(\Omega_{l}\right)$ в ортогональную сумму (см. [10, с. 113]):

$$
\boldsymbol{L}_{2}\left(\Omega_{l}\right)=\boldsymbol{J}_{0}\left(\Omega_{l}\right) \oplus \boldsymbol{G}_{h, S_{l}}\left(\Omega_{l}\right) \oplus \boldsymbol{G}_{0, \widetilde{\Gamma}_{l}}\left(\Omega_{l}\right), \quad l=2,3,
$$

где

$$
\begin{aligned}
\boldsymbol{J}_{0}\left(\Omega_{l}\right) & :=\left\{\boldsymbol{u} \in \boldsymbol{L}_{2}\left(\Omega_{l}\right): \operatorname{div} \boldsymbol{u}=0 \text { в } \Omega_{l}, u_{n}=0 \text { на } \partial \Omega_{l}\right\}, \\
\boldsymbol{G}_{h, S_{l}}\left(\Omega_{l}\right) & :=\left\{\boldsymbol{v}=\nabla \varphi \in \boldsymbol{L}_{2}\left(\Omega_{l}\right): \Delta \varphi=0 \text { в } \Omega_{l}, \frac{\partial \varphi}{\partial n}=0 \text { на } S_{l}\right\}, \\
\boldsymbol{G}_{0, \widetilde{\Gamma}_{l}}\left(\Omega_{l}\right) & :=\left\{\boldsymbol{w}=\nabla \psi \in \boldsymbol{L}_{2}\left(\Omega_{l}\right): \Delta \psi=0 \text { в } \Omega_{l}, \psi=0 \text { на } \widetilde{\Gamma}_{l}\right\} .
\end{aligned}
$$

Здесь $\widetilde{\Gamma}_{2}:=\Gamma_{1} \cup \Gamma_{2}, \widetilde{\Gamma}_{3}:=\Gamma_{2}$.

Введем ортопроекторы на соответствующие подпространства: $P_{0, l}, P_{h, S_{l}}, P_{02, \widetilde{\Gamma}_{l}}, l=2,3$.

Основываясь на разложении (2.2.1), применим метод ортогонального проектирования к уравнениям движения начально-краевой задачи (2.1.2)-(2.1.11). В силу условия соленоидальности, условия непротекания на твердых стенках $S_{l}$ и условия сохранения объема на свободных границах $\Gamma_{j}, j=1,2$ (так как жидкости несжимаемы), считаем, что

$$
\boldsymbol{u}_{l} \in \boldsymbol{J}_{0}\left(\Omega_{l}\right) \oplus \boldsymbol{G}_{h, S_{l}}\left(\Omega_{l}\right)=: \boldsymbol{J}_{0, S_{l}}\left(\Omega_{l}\right), \quad l=2,3 .
$$

Поля $\nabla p_{l}, l=2,3$, потенциальны и поэтому

$$
\nabla p_{l} \in \boldsymbol{G}\left(\Omega_{l}\right)=\boldsymbol{G}_{h, S_{l}}\left(\Omega_{l}\right) \oplus \boldsymbol{G}_{0, \widetilde{\Gamma}_{l}}\left(\Omega_{l}\right), \quad l=2,3 .
$$

Представим поля $\boldsymbol{u}_{l}$ и $\nabla p_{l}, l=2,3$, в следующем виде:

$$
\begin{gathered}
\boldsymbol{u}_{l}=\boldsymbol{w}_{l}+\nabla \Phi_{l}, \quad \boldsymbol{w}_{l} \in \boldsymbol{J}_{0}\left(\Omega_{l}\right), \quad \nabla \Phi_{l} \in \boldsymbol{G}_{h, S_{l}}\left(\Omega_{l}\right), \quad l=2,3 ; \\
\nabla p_{l}=\nabla \tilde{p}_{l}+\nabla \varphi_{l}, \quad \nabla \tilde{p}_{l} \in \boldsymbol{G}_{h, S_{l}}\left(\Omega_{l}\right), \quad \nabla \varphi_{l} \in \boldsymbol{G}_{0, \widetilde{\Gamma}_{l}}\left(\Omega_{l}\right), \quad l=2,3 .
\end{gathered}
$$

Подставив эти представления в уравнения (2.1.4) для идеальных жидкостей и применив к ним ортопроекторы, отвечающие разложению (2.2.1), получим

$$
\begin{gathered}
\frac{\partial \boldsymbol{w}_{l}}{\partial t}=P_{0, l} \boldsymbol{f}_{l} \quad \text { в } \Omega_{l}, \quad l=2,3, \\
\rho_{l} \frac{\partial}{\partial t} \nabla \Phi_{l}=-\nabla \tilde{p}_{l}+\rho_{l} P_{h, S_{l}} \boldsymbol{f}_{l} \quad \text { в } \Omega_{l}, \quad l=2,3, \\
\nabla \varphi_{l}=\rho_{l} P_{0, \widetilde{\Gamma}_{l}} \boldsymbol{f}_{l} \quad \text { в } \Omega_{l}, \quad l=2,3 .
\end{gathered}
$$

Из (2.2.5) с учетом начальных условий (2.1.11) сразу получаем

$$
\boldsymbol{w}_{l}(t, x)=\int_{0}^{t} P_{0, l} \boldsymbol{f}_{l}(\tau, x) d \tau+P_{0, l} \boldsymbol{u}_{l}^{0}, \quad l=2,3 .
$$

Члены в уравнении (2.2.7) - это составляющие градиентов давлений в подпространстве $\boldsymbol{G}_{0, \widetilde{\Gamma}_{l}}\left(\Omega_{l}\right), l=2,3$. Потенциал этого поля $\varphi_{l}$ обращается в нуль на $\widetilde{\Gamma}_{l}$ и поэтому в граничных условиях не участвует. Далее соотношения (2.2.7) не рассматриваем. 
Условимся называть решения уравнений (2.2.5), а также составляющие градиентов давлений из (2.2.7) тривиалъными решениями. Итак, основные уравнения, которые будем рассматривать для идеальных жидкостей, это уравнения (2.2.6).

Проектирование уравнения Навъе-Cтокса. Для области $\Omega_{1}$ введем аналогичное разложение пространства векторных полей $\boldsymbol{L}_{2}\left(\Omega_{1}\right)$ в ортогональную сумму (см. [10], а также п. 1.4):

$$
\boldsymbol{L}_{2}\left(\Omega_{1}\right)=\boldsymbol{J}_{0, S_{1}}\left(\Omega_{1}\right) \oplus \boldsymbol{G}_{0, \Gamma_{1}}\left(\Omega_{1}\right), \quad \boldsymbol{J}_{0, S_{1}}\left(\Omega_{1}\right)=\boldsymbol{J}_{0}\left(\Omega_{1}\right) \oplus \boldsymbol{G}_{h, S_{1}}\left(\Omega_{1}\right) .
$$

Введем ортопроекторы $P_{0, S_{1}}$ и $P_{01, \Gamma_{1}}$ на подпространства $\boldsymbol{J}_{0, S_{1}}\left(\Omega_{1}\right)$ и $\boldsymbol{G}_{0, \Gamma_{1}}\left(\Omega_{1}\right)$ соответственно. В силу условия соленоидальности и условия прилипания на $S_{1}$ для $\boldsymbol{u}_{1}$ считаем, что поле $\boldsymbol{u}_{1}$ принадлежит пространству векторных полей с конечной скоростью диссипации энергии в жидкости:

$$
\boldsymbol{J}_{0, S_{1}}^{1}\left(\Omega_{1}\right):=\left\{\boldsymbol{u}_{1} \in \boldsymbol{H}^{1}\left(\Omega_{1}\right): \operatorname{div} \boldsymbol{u}_{1}=0 \text { в } \Omega_{1}, \boldsymbol{u}_{1}=\mathbf{0} \text { на } S_{1}\right\} \subset \boldsymbol{J}_{0, S_{1}}\left(\Omega_{1}\right) .
$$

Здесь скалярное произведение определяется по формуле

$$
\left(\boldsymbol{u}_{1}, \boldsymbol{v}_{1}\right)_{\boldsymbol{J}_{0, S_{1}}^{1}\left(\Omega_{1}\right)}:=E_{1}\left(\boldsymbol{u}_{1}, \boldsymbol{v}_{1}\right)=\frac{1}{2} \int_{\Omega_{1}}\left(\sum_{q, r=1}^{3} \tau_{q r}\left(\boldsymbol{u}_{1}\right) \overline{\tau_{q r}\left(\boldsymbol{v}_{1}\right)}\right) d \Omega_{1}
$$

(см. $(1.2 .12),(2.1 .12))$. Отметим, что $\boldsymbol{J}_{0, S_{1}}^{1}\left(\Omega_{1}\right)$ плотно вложено в $\boldsymbol{J}_{0, S_{1}}\left(\Omega_{1}\right)$. Подействуем введенными операторами $P_{0, S_{1}}$ и $P_{01, \Gamma_{1}}$ на обе части уравнения для вязкоупругой жидкости (2.1.2); в результате получим:

$$
\begin{gathered}
\rho_{1} \frac{\partial \boldsymbol{u}_{1}}{\partial t}=-\nabla \tilde{p}_{1}+\mu_{1} P_{0, S_{1}} \Delta \boldsymbol{v}_{1}+\rho_{1} P_{0, S_{1}} \boldsymbol{f}_{1} \quad \text { в } \Omega_{1}, \\
-\mu_{1} P_{01, \Gamma_{1}} \Delta \boldsymbol{v}_{1}+P_{01, \Gamma_{1}} \nabla p_{1}=\rho_{1} P_{01, \Gamma_{1}} \boldsymbol{f}_{1} \quad \text { в } \Omega_{1} .
\end{gathered}
$$

Здесь через $\nabla \tilde{p}_{1}$ обозначено поле $P_{0, S_{1}} \nabla p_{1}=: \nabla \tilde{p}_{1} \in \boldsymbol{G}_{h, S_{1}}\left(\Omega_{1}\right)$ в силу разложения пространства $\boldsymbol{L}_{2}\left(\Omega_{1}\right)$. Потенциал поля $P_{01, \Gamma_{1}} \nabla p_{1}$ в граничных условиях не участвует, так как обращается в нуль на $\Gamma_{1}$. Поэтому для вязкоупругой жидкости рассматриваем только уравнение $(2.2 .8)$.

Формулировка задачи после отделения тривиалъных решений. Заметим, что в силу представлений (2.2.3) полей $\boldsymbol{u}_{l}, l=2,3$, кинематические условия (2.1.8) можно записать в виде

$$
\frac{\partial \zeta_{j}}{\partial t}=\gamma_{n, j} \boldsymbol{u}_{j}=\frac{\partial \Phi_{j+1}}{\partial n_{j}} \quad \text { на } \Gamma_{j}, \quad j=1,2
$$

В свою очередь динамические условия (2.1.10) в силу представлений (2.2.4) для $\nabla p_{l}, l=2,3$, примут вид

$$
\begin{aligned}
\mu_{1} \tau_{q 3}\left(\boldsymbol{v}_{1}\right)=0, \quad q=1,2 & \text { на } \Gamma_{1}, \\
{\left[-\tilde{p}_{1}+\mu_{1} \tau_{33}\left(\boldsymbol{v}_{1}\right)\right]-\left[-\tilde{p}_{2}\right]=-g\left(\rho_{1}-\rho_{2}\right) \zeta_{1} } & \text { на } \Gamma_{1}, \\
{\left[\tilde{p}_{2}-\tilde{p}_{3}\right]=g\left(\rho_{2}-\rho_{3}\right) \zeta_{2} } & \text { на } \Gamma_{2} .
\end{aligned}
$$

Введем еще ортопроекторы $\theta_{j}$ на $L_{2, \Gamma_{j}}:=L_{2}\left(\Gamma_{j}\right) \ominus\left\{1_{\Gamma_{j}}\right\}, j=1,2$. Тогда итогом проведенных выше преобразований является следующая теорема.

Теорема 2.1. Пусть $\boldsymbol{u}_{k}, \nabla p_{k}, k=\overline{1,3}, \zeta_{j}, j=1,2,-$ классическое решение начально-краевой задачи (2.1.2)-(2.1.11). Тогда функиии $\boldsymbol{u}_{1}, \nabla \tilde{p}_{1}, \nabla \Phi_{l}, \nabla \tilde{p}_{l}, l=2,3, \zeta_{j}, j=1,2$, являются решением следующей начально-краевой задачи:

$$
\begin{aligned}
& \rho_{1} \frac{\partial \boldsymbol{u}_{1}}{\partial t}=-\nabla \tilde{p}_{1}+\mu_{1} P_{0, S_{1}} \Delta \boldsymbol{v}_{1}+\rho_{1} P_{0, S_{1}} \boldsymbol{f}_{1} \quad{ }_{8} \Omega_{1}, \\
& \operatorname{div} \boldsymbol{u}_{1}=0 \quad \text { в } \Omega_{1}, \quad \boldsymbol{u}_{1}=\mathbf{0} \quad \text { на } S_{1}, \\
& \rho_{l} \frac{\partial}{\partial t} \nabla \Phi_{l}+\nabla \tilde{p}_{l}=\rho_{l} P_{h, S_{l}} \boldsymbol{f}_{l} \quad{ }_{8} \Omega_{l}, \quad l=2,3, \\
& \Delta \Phi_{l}=0 \quad \text { в } \Omega_{l}, \quad \frac{\partial \Phi_{l}}{\partial n_{l}}=0 \quad \text { на } S_{l},
\end{aligned}
$$




$$
\begin{aligned}
& \frac{\partial \Phi_{l}}{\partial n_{l}}=-\frac{\partial \zeta_{l-1}}{\partial t}=-\gamma_{n, l-1} \boldsymbol{u}_{l-1} \quad \text { на } \Gamma_{l-1}, \quad l=2,3, \\
& \left\{\begin{aligned}
\mu_{1} \tau_{q 3}\left(\boldsymbol{v}_{1}\right)=0, \quad q=1,2, & \text { на } \Gamma_{1}, \\
{\left[-\theta_{1} \tilde{p}_{1}+\mu_{1} \tau_{33}\left(\boldsymbol{v}_{1}\right)\right]-\left[-\theta_{1} \tilde{p}_{2}\right]=-g\left(\rho_{1}-\rho_{2}\right) \zeta_{1} } & \text { на } \Gamma_{1}, \\
{\left[\theta_{2} \tilde{p}_{2}-\theta_{2} \tilde{p}_{3}\right]=g\left(\rho_{2}-\rho_{3}\right) \zeta_{2} } & \text { на } \Gamma_{2},
\end{aligned}\right. \\
& \boldsymbol{u}_{1}(0, x)=P_{0, S_{1}} \boldsymbol{u}_{1}^{0}(x)=\boldsymbol{u}_{1}^{0}(x), \quad x \in \Omega_{1}, \quad \nabla \Phi_{l}(0, x)=P_{h, S_{l}} \boldsymbol{u}_{l}^{0}(x), \quad x \in \Omega_{l}, \quad l=2,3, \\
& \zeta_{j}(0, x)=\zeta_{j}^{0}(x), \quad x \in \Gamma_{j}, \quad j=1,2 .
\end{aligned}
$$

Замечание 2.1. Отметим, что введение ортопроекторов $\theta_{j}, j=1,2$, обусловлено стремлением избежать произвола в нормировке функций $\tilde{p}_{2}$ на границах $\Gamma_{1}$ и $\Gamma_{2}$. Действительно, в динамических условиях $(2.2 .9)$ имеем $\zeta_{j} \in L_{2, \Gamma_{j}}, j=1,2, \tau_{33}\left(\boldsymbol{v}_{1}\right) \in L_{2, \Gamma_{1}}$ (см. [10, с. 114]), откуда следует, что

$$
\int_{\Gamma_{1}}\left(\tilde{p}_{1}-\tilde{p}_{2}\right) d \Gamma_{1}=0, \quad \int_{\Gamma_{2}}\left(\tilde{p}_{2}-\tilde{p}_{3}\right) d \Gamma_{2}=0 .
$$

Из определения ортопроекторов $\theta_{j}, j=1,2$, следует, что $\tilde{p}_{1}=\theta_{1} \tilde{p}_{1}+c_{1}, \tilde{p}_{3}=\theta_{2} \tilde{p}_{3}+c_{3}$. Константы $c_{1}, c_{3}$ позволяют ввести однозначную нормировку для $\tilde{p}_{2}$ на границах $\Gamma_{1}$ и $\Gamma_{2}$ :

$$
c_{1}=\frac{1}{\left|\Gamma_{1}\right|} \int_{\Gamma_{1}} \tilde{p}_{2} d \Gamma_{1}, \quad c_{3}=\frac{1}{\left|\Gamma_{2}\right|} \int_{\Gamma_{2}} \tilde{p}_{2} d \Gamma_{2} .
$$

Вспомогателъные краевые задачи $u$ их операторы. Как и в разделе 1, считаем, что области $\Omega_{k}$ имеют липшицевы границы $\partial \Omega_{k}, k=\overline{1,3}$, разбитые на липшицевы куски. Для перехода к операторной формулировке исследуемой задачи рассмотрим ряд вспомогательных краевых задач.

Вспомогательная задача I:

$$
\begin{gathered}
-P_{0, S_{1}} \Delta \boldsymbol{u}+\mu^{-1} \nabla p=\boldsymbol{f}, \quad \operatorname{div} \boldsymbol{u}=0 \quad \text { в } \Omega_{1}, \quad \boldsymbol{u}=\mathbf{0} \quad \text { на } S_{1}, \\
\mu \tau_{q 3}(\boldsymbol{u})=0, \quad q=1,2, \quad-p+\mu \tau_{33}(\boldsymbol{u})=0 \quad \text { на } \Gamma_{1} .
\end{gathered}
$$

Это так называемая первая вспомогательная задача С. Г. Крейна (см. [10, с. 116], а также (1.4.4)). Она имеет единственное обобщенное решение $\boldsymbol{u}=A_{1}^{-1} \boldsymbol{f}$ для любого вектора $\boldsymbol{f}$ из $\boldsymbol{J}_{0, S_{1}}\left(\Omega_{1}\right)$. Область определения $\mathcal{D}\left(A_{1}\right)$ оператора $A_{1}$ плотна в пространстве $\boldsymbol{J}_{0, S_{1}}^{1}\left(\Omega_{1}\right)$ и $\mathcal{D}\left(A_{1}^{1 / 2}\right)=\boldsymbol{J}_{0, S_{1}}^{1}\left(\Omega_{1}\right)$. Оператор $A_{1}^{-1}$ является положительным и компактным в $\boldsymbol{J}_{0, S_{1}}\left(\Omega_{1}\right)$.

Вспомогательная задача II:

$$
\Delta p=0 \quad \text { в } \Omega_{1}, \quad \frac{\partial p}{\partial n_{1}}=0 \quad \text { на } S_{1}, \quad p=\tau_{1} \quad \text { на } \Gamma_{1}, \quad \int_{\Gamma_{1}} \tau_{1} d \Gamma_{1}=0 .
$$

Это известная задача Зарембы для уравнения Лапласа; она имеет единственное решение (см. [10, c. 45]) $p=G_{1} \tau_{1} \in H_{\Gamma_{1}}^{1}\left(\Omega_{1}\right)$ при $\tau_{1} \in H_{\Gamma_{1}}^{1 / 2}$,

$$
H_{\Gamma_{1}}^{1}\left(\Omega_{1}\right):=\left\{\varphi \in H^{1}\left(\Omega_{1}\right): \int_{\Gamma_{1}} \varphi d \Gamma_{1}=0\right\}, \quad\|\varphi\|_{H_{\Gamma_{1}}^{1}\left(\Omega_{1}\right)}^{2}:=\int_{\Omega_{1}}|\nabla \varphi|^{2} d \Omega_{1} .
$$

(Определение пространств $H_{\partial \Omega}^{1 / 2}, H_{\partial \Omega}^{-1 / 2}$ для областей $\Omega$ с липшицевой границей $\partial \Omega$, а также соответствующие теоремы вложения и продолжения с границы см. в [21], а также в п. 1.3.)

Вспомогательная задача III:

$$
\Delta \Phi=0 \quad \text { в } \Omega_{3}, \quad \frac{\partial \Phi}{\partial n_{3}}=0 \quad \text { на } S_{3}, \quad \frac{\partial \Phi}{\partial n_{3}}=\psi_{3} \quad \text { на } \Gamma_{2}, \quad \int_{\Gamma_{2}} \Phi d \Gamma_{2}=\int_{\Gamma_{2}} \psi_{3} d \Gamma_{2}=0 .
$$


Это задача Неймана для уравнения Лапласа. Если $\psi_{3} \in \tilde{H}_{\Gamma_{2}}^{-1 / 2}$, то задача имеет единственное решение $\Phi=V_{3} \psi_{3} \in H_{\Gamma_{2}}^{1}\left(\Omega_{3}\right)$. Здесь символом обозначен класс функций из $H_{\Gamma_{2}}^{-1 / 2}$, продолжимых нулем на всю границу $\partial \Omega_{3}$ в классе $H^{-1 / 2}\left(\partial \Omega_{3}\right)$ (см. $\left.[1,8,19]\right)$. Введем по решению задачи III оператор

$$
\left.\theta_{2} \Phi\right|_{\Gamma_{2}}=\theta_{2} \hat{C}_{3} \psi_{3}=: C_{3} \psi_{3}, \quad \hat{C}_{3}:=\gamma_{\Gamma_{2}} V_{3}, \quad \gamma_{\Gamma_{2}} \psi_{3}:=\left.\psi_{3}\right|_{\Gamma_{2}} .
$$

Отметим, что $C_{3}$ - самосопряженный, положительный и компактный оператор в $L_{2, \Gamma_{2}}$.

Вспомогательная задача IV.1:

$$
\begin{gathered}
\Delta \Phi_{21}=0 \quad \text { в } \Omega_{2}, \quad \frac{\partial \Phi_{21}}{\partial n_{2}}=0 \quad \text { на } S_{2} \cup \Gamma_{2}, \quad \frac{\partial \Phi_{21}}{\partial n_{2}}=\psi_{1} \quad \text { на } \Gamma_{1}, \\
\int_{\Gamma_{1}} \Phi_{21} d \Gamma_{1}=\int_{\Gamma_{1}} \psi d \Gamma_{1}=0 .
\end{gathered}
$$

Это снова задача Неймана для уравнения Лапласа. Если $\psi_{1} \in \tilde{H}_{\Gamma_{1}}^{-1 / 2}$, то задача имеет единственное решение $\Phi_{21}=V_{21} \psi_{1} \in H_{\Gamma_{1}}^{1}\left(\Omega_{2}\right)$.

Вспомогательная задача IV.2:

$$
\begin{gathered}
\Delta \Phi_{22}=0 \quad \text { в } \Omega_{2}, \quad \frac{\partial \Phi_{22}}{\partial n_{2}}=0 \quad \text { на } S_{2} \cup \Gamma_{1}, \quad \frac{\partial \Phi_{22}}{\partial n_{2}}=\psi_{2} \quad \text { на } \Gamma_{2}, \\
\int_{\Gamma_{2}} \Phi_{22} d \Gamma_{2}=\int_{\Gamma_{2}} \psi d \Gamma_{2}=0 .
\end{gathered}
$$

Это тоже задача Неймана для уравнения Лапласа. Если $\psi_{2} \in \tilde{H}_{\Gamma_{2}}^{-1 / 2}$, то задача имеет единственное решение $\Phi_{22}=V_{22} \psi_{2} \in H_{\Gamma_{2}}^{1}\left(\Omega_{2}\right)$.

Введем по решению задач IV.1 и IV.2 операторы

$$
\begin{array}{lll}
\left.\theta_{1} \Phi_{21}\right|_{\Gamma_{1}}=\theta_{1} \hat{C}_{11} \psi_{1}=: C_{11} \psi_{1}, & \hat{C}_{11}:=\gamma_{\Gamma_{1}} V_{21}, & \gamma_{\Gamma_{1}} \psi_{1}:=\left.\psi_{1}\right|_{\Gamma_{1}} \\
\left.\theta_{1} \Phi_{22}\right|_{\Gamma_{1}}=\theta_{1} \hat{C}_{12} \psi_{2}=: C_{12} \psi_{2}, & \hat{C}_{12}:=\gamma_{\Gamma_{1}} V_{22}, & \gamma_{\Gamma_{1}} \psi_{2}:=\left.\psi_{2}\right|_{\Gamma_{1}} ; \\
\left.\theta_{2} \Phi_{21}\right|_{\Gamma_{2}}=\theta_{2} \hat{C}_{21} \psi_{1}=: C_{21} \psi_{1}, & \hat{C}_{21}:=\gamma_{\Gamma_{2}} V_{21}, & \gamma_{\Gamma_{2}} \psi_{1}:=\left.\psi_{1}\right|_{\Gamma_{2}} \\
\left.\theta_{2} \Phi_{22}\right|_{\Gamma_{2}}=\theta_{2} \hat{C}_{22} \psi_{2}=: C_{22} \psi_{2}, & \hat{C}_{22}:=\gamma_{\Gamma_{2}} V_{22}, & \gamma_{\Gamma_{2}} \psi_{2}:=\left.\psi_{2}\right|_{\Gamma_{2}}
\end{array}
$$

Отметим, что компактные операторы $C_{q r}, q, r=1,2$, образуют самосопряженную, положительную матрицу $\left\{C_{q r}\right\}_{q, r=1}^{2}$ в $L_{2, \Gamma_{1}} \oplus L_{2, \Gamma_{2}}$ (см., например, [8]).

Вспомогательная задача V:

$$
\Delta p=0 \quad \text { в } \Omega_{2}, \quad \frac{\partial p}{\partial n_{2}}=0 \quad \text { на } S_{2} \cap \Gamma_{1}, \quad p=\tau_{2} \quad \text { на } \Gamma_{2}, \quad \int_{\Gamma_{2}} \tau_{2} d \Gamma_{2}=0 .
$$

Это, как и II, задача Зарембы для уравнения Лапласа. Она имеет единственное решение (см. [10, c. 45]) $p=G_{2} \tau_{2} \in H_{\Gamma_{2}}^{1}\left(\Omega_{2}\right)$ при $\tau_{2} \in H_{\Gamma_{2}}^{1 / 2}$.

Вывод системы операторных уравнений. Переходя к формулировке исходной задачи (2.1.2)-(2.1.11) в операторной форме, представим поле $\nabla \tilde{p}_{1}$ в виде $\nabla \tilde{p}_{1}=\nabla \tilde{p}_{11}+\nabla \tilde{p}_{12}$ и подберем поле $\nabla \tilde{p}_{11}$ таким образом, чтобы поле $\boldsymbol{v}_{1}$ являлось решением следующей краевой задачи:

$$
\begin{gathered}
-P_{0, S_{1}} \Delta \boldsymbol{v}_{1}+\mu_{1}^{-1} \nabla \tilde{p}_{11}=\mu_{1}^{-1}\left(-\rho_{1} \frac{\partial \boldsymbol{u}_{1}}{\partial t}+\rho_{1} P_{0, S_{1}} \boldsymbol{f}_{1}-\nabla \tilde{p}_{12}\right) \quad \text { в } \Omega_{1}, \\
\operatorname{div} \boldsymbol{v}_{1}=0 \quad \text { в } \Omega_{1}, \quad \boldsymbol{v}_{1}=\mathbf{0} \quad \text { на } S_{1}, \\
\mu_{1} \tau_{q 3}\left(\boldsymbol{v}_{1}\right)=0, \quad q=1,2, \quad-\theta_{1} \tilde{p}_{11}+\mu \tau_{33}\left(\boldsymbol{v}_{1}\right)=0 \quad \text { на } \Gamma_{1} .
\end{gathered}
$$


Используя вспомогательную задачу I, заключаем, что краевая задача (2.2.18)-(2.2.20) имеет единственное обобщенное решение

$$
\boldsymbol{v}_{1}=\mu_{1}^{-1} A_{1}^{-1}\left(-\rho_{1} \frac{\partial \boldsymbol{u}_{1}}{\partial t}+\rho_{1} P_{0, S_{1}} \boldsymbol{f}_{1}-\nabla \tilde{p}_{12}\right)
$$

для правой части из $\boldsymbol{J}_{0, S_{1}}\left(\Omega_{1}\right)$. Тогда можно записать

$$
\rho_{1} \frac{\partial \boldsymbol{u}_{1}}{\partial t}+\mu_{1} A_{1} \boldsymbol{v}_{1}+\nabla \tilde{p}_{12}=\rho_{1} P_{0, S_{1}} \boldsymbol{f}_{1} \quad \text { в } \Omega_{1} .
$$

Заметим, что при расщеплении условия для нормального напряжения на $\Gamma_{1}$ из (2.2.15) осталось условие

$$
\theta_{1} \tilde{p}_{12}=\theta_{1} \tilde{p}_{2}+g\left(\rho_{1}-\rho_{2}\right) \zeta_{1} \quad \text { на } \Gamma_{1} \text {. }
$$

Учитывая принадлежность $\nabla \tilde{p}_{12} \in \boldsymbol{G}_{h, S_{1}}\left(\Omega_{1}\right)$, найдем, что потенциал $\theta_{1} \tilde{p}_{12}$ удовлетворяет вспомогательной задаче II при $\tau_{1}=\theta_{1} \tilde{p}_{2}+g\left(\rho_{1}-\rho_{2}\right) \zeta_{1}$. Поэтому можно считать, что

$$
\tilde{p}_{12}=G_{1}\left(\theta_{1} \tilde{p}_{2}+g\left(\rho_{1}-\rho_{2}\right) \zeta_{1}\right) .
$$

Оператор $G_{1}$ ограниченно действует из пространства $H_{\Gamma_{1}}^{1 / 2}$ в пространство $\boldsymbol{G}_{h, S_{1}}\left(\Omega_{1}\right)$.

Подставляя последнее представление для градиента давления в (2.2.21), получим

$$
\rho_{1} \frac{\partial \boldsymbol{u}_{1}}{\partial t}+\mu_{1} A_{1} \boldsymbol{v}_{1}+G_{1} \theta_{1} \tilde{p}_{2}+g\left(\rho_{1}-\rho_{2}\right) G_{1} \zeta_{1}=\rho_{1} P_{0, S_{1}} \boldsymbol{f}_{1} \quad \text { в } \Omega_{1} .
$$

Далее, в силу принадлежности $\nabla \Phi_{2}$ пространству $\boldsymbol{G}_{h, S_{2}}\left(\Omega_{2}\right)$ потенциал $\Phi_{2}$ с помощью решений вспомогательных задач IV.1 и IV.2 можно представить в виде

$$
\Phi_{2}=\Phi_{21}+\Phi_{22} \quad \text { при } \quad \psi_{1}=-\gamma_{n, 1} \boldsymbol{u}_{1}, \quad \psi_{2}=\gamma_{n, 2} \boldsymbol{u}_{2} .
$$

Рассмотрим уравнения (2.2.12) для идеальных жидкостей. Из них следуют интегралы КошиЛагранжа

$$
\rho_{l} \frac{\partial \Phi_{l}}{\partial t}+\tilde{p}_{l}=F_{l}+c_{l}(t) \quad \text { в } \Omega_{l}, \quad l=2,3,
$$

где через $F_{l}$ обозначены потенциалы полей $\rho_{l} P_{h, S_{l}} \boldsymbol{f}_{l}$, а $c_{l}(t)$ - произвольные функции переменной $t, l=2,3$. Рассмотрим эти уравнения на $\Gamma_{2}$ и спроектируем на $L_{2, \Gamma_{2}}$; тогда динамическое условие (см. третье уравнение (2.2.15)) можно преобразовать к следующему виду:

$$
\theta_{2} \tilde{p}_{2}=\theta_{2} \tilde{p}_{3}+g\left(\rho_{2}-\rho_{3}\right) \zeta_{2}=\rho_{3} \theta_{2} F_{3}+\rho_{3} \theta_{2} \frac{\partial \Phi_{3}}{\partial t}+g\left(\rho_{2}-\rho_{3}\right) \zeta_{2} \quad \text { на } \Gamma_{2} .
$$

Выразим теперь $\left.\theta_{2} \Phi_{3}\right|_{\Gamma_{2}}$ с помощью представления (2.2.17) и оператора $C_{3}$ :

$$
\left.\theta_{2} \Phi_{3}\right|_{\Gamma_{2}}=-C_{3} \gamma_{n, 2} \boldsymbol{u}_{2} \quad \text { на } \Gamma_{2} .
$$

Тогда вместо (2.2.24) получим

$$
\theta_{2} \tilde{p}_{2}=\rho_{3} \theta_{2} F_{3}-\rho_{3} \frac{\partial}{\partial t} C_{3} \gamma_{n, 2} \boldsymbol{u}_{2}+g\left(\rho_{2}-\rho_{3}\right) \zeta_{2} \quad \text { на } \Gamma_{2} .
$$

Значит, согласно вспомогательной задаче $\mathrm{V}$,

$$
\left.\tilde{p}_{2}\right|_{\Omega_{2}}=G_{2}\left(\rho_{3} \theta_{2} F_{3}-\rho_{3} \frac{\partial}{\partial t} C_{3} \gamma_{n, 2} \boldsymbol{u}_{2}+g\left(\rho_{2}-\rho_{3}\right) \zeta_{2}\right) .
$$

Выразим при $l=2$ функцию $\tilde{p}_{2}$ из (2.2.23); тогда уравнение $(2.2 .22)$ можно переписать в виде

$$
\begin{aligned}
\rho_{1} \frac{\partial \boldsymbol{u}_{1}}{\partial t}+\mu_{1} A_{1} \boldsymbol{v}_{1}+G_{1} \theta_{1}\left(\rho_{2} F_{2}-\rho_{2} \frac{\partial}{\partial t}\left(C_{11} \gamma_{n, 1} \boldsymbol{u}_{1}+C_{12} \gamma_{n, 2} \boldsymbol{u}_{2}\right)\right)+g\left(\rho_{1}-\rho_{2}\right) G_{1} \zeta_{1}= & =\rho_{1} P_{0, S_{1}} \boldsymbol{f}_{1} \quad \text { в } \Omega_{1} .
\end{aligned}
$$

Далее для всех функций переменной $t$ со значениями в гильбертовых пространствах их производные по $t$ будем обозначать $d / d t$ вместо $\partial / \partial t$.

Проведенные в этом пункте рассуждения можно сформулировать в виде следующей леммы. 
Лемма 2.1. Пусть $\boldsymbol{u}_{1}, \nabla \tilde{p}_{1}, \nabla \Phi_{l}, \nabla \tilde{p}_{l}, l=2,3, \zeta_{j}, j=1,2,-$ классическое решение начальнокраевой задачи (2.2.10)-(2.2.16). Тогда функиии $\boldsymbol{u}_{k}, k=1,2, \zeta_{j}, j=1,2$ являются решением следующей задачи Коши:

$$
\begin{aligned}
& \begin{aligned}
\frac{d}{d t}\left(\rho_{1} \boldsymbol{u}_{1}+\rho_{2}\left(G_{1} C_{11} \gamma_{n, 1} \boldsymbol{u}_{1}+G_{1} C_{12} \gamma_{n, 2} \boldsymbol{u}_{2}\right)\right)+\mu_{1} A_{1} \boldsymbol{v}_{1} & +g\left(\rho_{1}-\rho_{2}\right) G_{1} \zeta_{1}= \\
& =\rho_{1} P_{0, S_{1}} \boldsymbol{f}_{1}-\rho_{2} G_{1} \theta_{1} F_{2},
\end{aligned} \\
& \begin{array}{r}
\frac{d}{d t}\left(\rho_{2}\left(G_{2} C_{21} \gamma_{n, 1} \boldsymbol{u}_{1}+G_{2} C_{22} \gamma_{n, 2} \boldsymbol{u}_{2}\right)+\rho_{3} G_{2} C_{3} \gamma_{n, 2} \boldsymbol{u}_{2}\right)+g\left(\rho_{2}-\rho_{3}\right) G_{2} \zeta_{2}= \\
=\rho_{2} G_{2} \theta_{2} F_{2}-\rho_{3} G_{2} \theta_{2} F_{3},
\end{array} \\
& \begin{aligned}
\frac{d}{d t}\left(g\left(\rho_{1}-\rho_{2}\right) \zeta_{1}\right)=g\left(\rho_{1}-\rho_{2}\right) \gamma_{n, 1} \boldsymbol{u}_{1}, \quad \frac{d}{d t}\left(g\left(\rho_{2}-\rho_{3}\right) \zeta_{2}\right)=g\left(\rho_{2}-\rho_{3}\right) \gamma_{n, 2} \boldsymbol{u}_{2}, \\
\boldsymbol{u}_{k}(0, x)=\boldsymbol{u}_{k}^{0}(x), \quad x \in \Omega_{k}, \quad k=1,2, \quad \zeta_{j}(0, x)=\zeta_{j}^{0}(x), \quad x \in \Gamma_{j}, \quad j=1,2 .
\end{aligned}
\end{aligned}
$$

Введем операторы $\hat{G}_{1}:=g\left(\rho_{1}-\rho_{2}\right) G_{1}, \hat{G}_{2}:=g\left(\rho_{2}-\rho_{3}\right) G_{2}$. Справедлива следующая лемма.

Лемма 2.2. Имеют место соотношения

$$
\begin{aligned}
& \hat{G}_{1}^{*}=g\left(\rho_{1}-\rho_{2}\right) \gamma_{n, 1}, \quad \gamma_{n, 1} \in \mathcal{L}\left(\boldsymbol{J}_{0, S_{1}}\left(\Omega_{1}\right) ; \tilde{H}_{\Gamma_{1}}^{-1 / 2}\right), \\
& \hat{G}_{2}^{*}=g\left(\rho_{2}-\rho_{3}\right) \gamma_{n, 2}, \quad \gamma_{n, 2} \in \mathcal{L}\left(\boldsymbol{J}_{0, S_{2}}\left(\Omega_{2}\right) ; \tilde{H}_{\Gamma_{2}}^{-1 / 2}\right) .
\end{aligned}
$$

Доказательство аналогично доказательству лемм 1.1 и 1.2 .

\section{3. Преобразование задачи к стандартному виду.}

Переход к системе обыкновенных дифференциальных уравнений. В задаче (2.2.25)(2.2.28) введем новые искомые функции по формулам

$$
\boldsymbol{w}_{r}(t):=\alpha_{r}^{1 / 2} \int_{0}^{t} e^{-\beta_{r}(t-s)} \boldsymbol{u}_{1}(s) d s \in \boldsymbol{J}_{0, S_{1}}^{1}\left(\Omega_{1}\right), \quad r=\overline{1, m} .
$$

Тогда

$$
\frac{d \boldsymbol{w}_{r}}{d t}=\alpha_{r}^{1 / 2} \boldsymbol{u}_{1}-\beta_{r} \alpha_{r}^{1 / 2} \int_{0}^{t} e^{-\beta_{r}(t-s)} \boldsymbol{u}_{1}(s) d s=\alpha_{r}^{1 / 2} \boldsymbol{u}_{1}-\beta_{r} \boldsymbol{w}_{r}, \quad r=\overline{1, m} .
$$

Теперь с учетом (2.3.1), (2.3.2) задача (2.2.25)-(2.2.28) переписывается в виде задачи Коши для системы обыкновенных дифференциальных уравнений первого порядка:

$$
\begin{gathered}
\frac{d}{d t}\left(\left(\rho_{1} I+\rho_{2} G_{1} C_{11} \gamma_{n, 1}\right) \boldsymbol{u}_{1}+\rho_{2} G_{1} C_{12} \gamma_{n, 2} \boldsymbol{u}_{2}\right)+\mu_{1} A_{1}\left(\boldsymbol{u}_{1}+\sum_{r=1}^{m} \alpha_{r} \boldsymbol{w}_{r}\right)+g\left(\rho_{1}-\rho_{2}\right) G_{1} \zeta_{1}= \\
=\rho_{1} P_{0, S_{1}} \boldsymbol{f}_{1}-\rho_{2} G_{1} F_{2} \\
\frac{d \boldsymbol{w}_{r}}{d t}-\alpha_{r}^{1 / 2} \boldsymbol{u}_{1}+\beta_{r} \boldsymbol{w}_{r}=0, \quad r=\overline{1, m}, \\
\frac{d}{d t}\left(\rho_{2} G_{2} C_{21} \gamma_{n, 1} \boldsymbol{u}_{1}+\left(\rho_{2} G_{2} C_{22} \gamma_{n, 2}+\rho_{3} G_{2} C_{3} \gamma_{n, 2}\right) \boldsymbol{u}_{2}\right)+g\left(\rho_{2}-\rho_{3}\right) G_{2} \zeta_{2}=\rho_{2} G_{2} F_{2}-\rho_{3} G_{2} F_{3}, \\
\frac{d}{d t}\left(g\left(\rho_{1}-\rho_{2}\right) \zeta_{1}\right)-\hat{G}_{1}^{*} \boldsymbol{u}_{1}=0, \quad \frac{d}{d t}\left(g\left(\rho_{2}-\rho_{3}\right) \zeta_{2}\right)-\hat{G}_{2}^{*} \boldsymbol{u}_{2}=0, \\
\boldsymbol{u}_{k}(0, x)=\boldsymbol{u}_{k}^{0}(x), \quad x \in \Omega_{k}, \quad k=1,2, \quad \zeta_{j}(0, x)=\zeta_{j}^{0}(x) \quad x \in \Gamma_{j}, \quad j=1,2, \\
\boldsymbol{w}_{r}(0, x)=\mathbf{0}, \quad r=\overline{1, m} .
\end{gathered}
$$


Коротко эта задача может быть записана в следующем виде:

$$
\mathcal{T} \frac{d z}{d t}=-\mathcal{A} z+g(t), \quad z(0)=z^{0}
$$

где $\mathcal{T}:=\operatorname{diag}\{\mathcal{C}, \mathcal{B}\}$

$$
\begin{gathered}
\mathcal{C}:=\left(\begin{array}{ccc}
\rho_{1} I+\rho_{2} G_{1} C_{11} \gamma_{n, 1} & \hat{0} & \rho_{2} G_{1} C_{12} \gamma_{n, 2} \\
\hat{0} & \hat{I} & \hat{0} \\
\rho_{2} G_{2} C_{21} \gamma_{n, 1} & 0 & \rho_{2} G_{2} C_{22} \gamma_{n, 2}+\rho_{3} G_{2} C_{3} \gamma_{n, 2}
\end{array}\right), \\
\mathcal{B}:=\operatorname{diag}\left\{g\left(\rho_{2}-\rho_{1}\right) I, g\left(\rho_{3}-\rho_{2}\right) I\right\} \\
\mathcal{A}:=\left(\begin{array}{cc}
\mathcal{A}_{0} & \mathcal{G} \\
-\mathcal{G}^{*} & \mathcal{O}
\end{array}\right), \quad \mathcal{A}_{0}=\left(\begin{array}{ccc}
A & \hat{\alpha} & 0 \\
-\hat{\alpha} & \hat{\beta} & \hat{0} \\
0 & 0 & 0
\end{array}\right), \quad \mathcal{G}=\left(\begin{array}{cc}
g\left(\rho_{2}-\rho_{1}\right) G_{1} & 0 \\
\hat{0} & \hat{0} \\
0 & g\left(\rho_{3}-\rho_{2}\right) G_{2}
\end{array}\right), \\
\mathcal{O}=\left(\begin{array}{ll}
0 & 0 \\
0 & 0
\end{array}\right), \quad z=\left(\begin{array}{c}
\boldsymbol{u}_{1} \\
\hat{y} \\
\boldsymbol{u}_{2} \\
\zeta_{1} \\
\zeta_{2}
\end{array}\right), \quad g(t)=\left(\begin{array}{c}
\rho_{1} P_{0, S_{1}} \boldsymbol{f}_{1}-\rho_{2} G_{1} F_{2} \\
0 \\
\rho_{2} G_{2} F_{2}-\rho_{3} G_{2} F_{3} \\
0 \\
0
\end{array}\right)
\end{gathered}
$$

Здесь

$$
\begin{gathered}
A:=\mu_{1} A_{1}, \quad \hat{\alpha}:=\left(\alpha_{1}^{1 / 2}, \alpha_{2}^{1 / 2}, \ldots, \alpha_{m}^{1 / 2}\right)^{\tau}, \quad \hat{\beta}:=\operatorname{diag}\left\{\beta_{1} I, \beta_{2} I, \ldots, \beta_{m} I\right\}, \quad \boldsymbol{u}_{2}=\nabla \Phi_{22}, \\
\hat{y}:=\left(A^{1 / 2} \boldsymbol{w}_{1}, A^{1 / 2} \boldsymbol{w}_{2}, \ldots, A^{1 / 2} \boldsymbol{w}_{m}\right)^{\tau}, \quad \hat{0}:=(\underbrace{0,0, \ldots, 0}_{m \text { раз }})^{\tau}, \quad \hat{I}_{m}:=\operatorname{diag}\{\underbrace{0,0, \ldots, 0}_{m \text { раз }}\} .
\end{gathered}
$$

Операторная матрица $\mathcal{A}$ задана на области определения

$$
\mathcal{D}(\mathcal{A})=\mathcal{D}(A) \oplus \hat{\mathcal{D}}\left(A^{1 / 2}\right) \oplus W \oplus H_{\Gamma_{1}}^{1 / 2} \oplus H_{\Gamma_{2}}^{1 / 2},
$$

где

$$
\hat{\mathcal{D}}\left(A^{1 / 2}\right):=\bigoplus_{r=1}^{m} \mathcal{D}\left(A^{1 / 2}\right)
$$

$$
W:=\left\{\nabla \Phi_{22} \in \boldsymbol{G}_{h, S_{2}, \Gamma_{2}}\left(\Omega_{2}\right):\left(\boldsymbol{u}_{1}, \nabla \Phi_{22}, \nabla \Phi_{3}\right)^{\tau} \in \boldsymbol{J}_{0, S}(\Omega), \boldsymbol{u}_{1} \in \mathcal{D}(A),\right.
$$

$$
\left.\boldsymbol{u}_{1} \cdot \boldsymbol{n}_{1}=-\nabla \Phi_{22} \cdot \boldsymbol{n}_{2} \in H_{\Gamma_{1}}^{1 / 2}, \nabla \Phi_{22} \cdot \boldsymbol{n}_{2}=-\nabla \Phi_{3} \cdot \boldsymbol{n}_{3} \in H_{\Gamma_{2}}^{1 / 2}\right\}
$$

и действует в пространстве

$$
\mathcal{H}:=\boldsymbol{J}_{0, S_{1}}\left(\Omega_{1}\right) \oplus \hat{\boldsymbol{J}}_{0, S_{1}}\left(\Omega_{1}\right) \oplus \boldsymbol{G}_{h, S_{2}, \Gamma_{2}}\left(\Omega_{2}\right) \oplus L_{2, \Gamma_{1}} \oplus L_{2, \Gamma_{2}},
$$

где

$$
\hat{\boldsymbol{J}}_{0, S_{1}}\left(\Omega_{1}\right):=\bigoplus_{r=1}^{m} \boldsymbol{J}_{0, S_{1}}\left(\Omega_{1}\right), \quad \boldsymbol{G}_{h, S_{2}, \Gamma_{2}}\left(\Omega_{2}\right):=\left\{\boldsymbol{v}=\nabla \varphi \in \boldsymbol{G}_{h, S_{2}}\left(\Omega_{2}\right): \frac{\partial \varphi}{\partial n}=0 \text { в } \Gamma_{1}\right\} .
$$

Замечание 2.2. Необходимо отметить, что задача (2.3.4) получена после дополнительной симметризации путем замены

$$
\boldsymbol{w}_{r}=A^{-1 / 2} \boldsymbol{y}_{r}, \quad \boldsymbol{y}_{r} \in \boldsymbol{J}_{0, S_{1}}\left(\Omega_{1}\right), \quad r=\overline{1, m} .
$$

Тогда из (2.3.3) имеем соотношения

$$
\frac{d}{d t}\left(A^{-1 / 2} \boldsymbol{y}_{r}\right)=\alpha_{r}^{1 / 2} \boldsymbol{u}_{1}-\beta_{r} A^{-1 / 2} \boldsymbol{y}_{r}, \quad r=\overline{1, m}
$$

и если $\boldsymbol{u}_{1}(t)$ - непрерывная по $t$ функция со значениями в $\boldsymbol{J}_{0, S_{1}}^{1}\left(\Omega_{1}\right)$, а $\boldsymbol{y}_{r}(t), r=\overline{1, m},-$ со значениями в $\boldsymbol{J}_{0, S_{1}}\left(\Omega_{1}\right)$, то правая часть в $(2.3 .10)$ непрерывна по $t$ со значениями в $\boldsymbol{J}_{0, S_{1}}^{1}\left(\Omega_{1}\right)=\mathcal{D}\left(A^{1 / 2}\right)$. Поэтому к обеим частям в $(2.3 .10)$ можно применить оператор $A^{1 / 2}$. 
Свойства операторных коэффициентов. Изучим свойства операторов задачи (2.3.4).

Лемма 2.3. Оператор $\mathcal{T}$ из (2.3.5)-(2.3.6) ограничен, самосопряжен и положительно определен.

Доказательство. Свойство ограниченности следует из того, что все операторные коэффициенты матрицы $\mathcal{T}$ ограничены. Чтобы в этом убедиться, достаточно проверить, например, ограниченность оператора $G_{1} C_{11} \gamma_{n, 1}$. Он действует из $\boldsymbol{J}_{0, S_{1}}\left(\Omega_{1}\right)$ в $\boldsymbol{G}_{h, S_{1}}\left(\Omega_{1}\right)$. Действительно, для $\boldsymbol{\xi} \in \boldsymbol{J}_{0, S_{1}}\left(\Omega_{1}\right)$ элемент $\gamma_{n, 1} \boldsymbol{\xi} \in \tilde{H}_{\Gamma_{1}}^{-1 / 2}$. С помощью вспомогательной задачи IV.1 найдем функцию $\Phi_{21}$ при условии, что $\psi_{1}=\gamma_{n, 1} \boldsymbol{\xi}$. Тогда в силу свойств ограниченности операторов $C_{11}$ и $G_{1}$ оператор $G_{1} C_{11} \gamma_{n, 1}$ ограничен, а его действие следующее:

$$
G_{1} C_{11} \gamma_{n, 1} \boldsymbol{\xi}=\left.G_{1} \theta_{1} \Phi_{21}\right|_{\Gamma_{1}} \in \boldsymbol{G}_{h, S_{1}}\left(\Omega_{1}\right) \subset \boldsymbol{J}_{0, S_{1}}\left(\Omega_{1}\right) .
$$

Найдем квадратичную форму оператора $\mathcal{T}$ в комплексном гильбертовом пространстве $\mathcal{H}$. Для любого $z \in \mathcal{H}$ имеем

$$
\begin{aligned}
&(\mathcal{T} z, z)_{\mathcal{H}}=\rho_{1} \int_{\Omega_{1}}\left|\boldsymbol{u}_{1}\right|^{2} d \Omega_{1}+\rho_{2} \int_{\Omega_{1}} G_{1} C_{11} \gamma_{n, 1} \boldsymbol{u}_{1} \cdot \overline{\boldsymbol{u}_{1}} d \Omega_{1}+ \\
&+\sum_{r=1}^{m} \int_{\Omega_{1}}\left|\boldsymbol{y}_{r}\right|^{2} d \Omega_{1}+\rho_{2} \int_{\Omega_{1}} G_{1} C_{12} \gamma_{n, 2} \nabla \Phi_{22} \cdot \overline{\boldsymbol{u}_{1}} d \Omega_{1}+\rho_{2} \int_{\Omega_{2}} G_{2} C_{21} \gamma_{n, 1} \boldsymbol{u}_{1} \cdot \overline{\nabla \Phi_{22}} d \Omega_{2}+ \\
& \quad+\rho_{2} \int_{\Omega_{2}} G_{2} C_{22} \gamma_{n, 2} \nabla \Phi_{22} \cdot \overline{\nabla \Phi_{22}} d \Omega_{2}+\rho_{3} \int_{\Omega_{2}} G_{2} C_{3} \gamma_{n, 2} \nabla \Phi_{22} \cdot \overline{\nabla \Phi_{22}} d \Omega_{1}+ \\
&+g\left(\rho_{2}-\rho_{1}\right) \int_{\Gamma_{1}}\left|\xi_{1}\right|^{2} d \Gamma_{1}+g\left(\rho_{3}-\rho_{2}\right) \int_{\Gamma_{2}}\left|\xi_{2}\right|^{2} d \Gamma_{2} .
\end{aligned}
$$

Преобразуем второе и четвертое слагаемые в (2.3.11), используя свойства решения вспомогательных задач IV.1, IV.2, операторов $C_{11}, C_{12}$, взаимную сопряженность операторов $G_{1}$ и $\gamma_{n, 1}$ и свойства потенциала $\Phi_{21}$ :

$$
\begin{aligned}
\rho_{2} \int_{\Omega_{1}} G_{1} C_{11} \gamma_{n, 1} \boldsymbol{u}_{1} \cdot \overline{\boldsymbol{u}_{1}} d \Omega_{1}+\rho_{2} \int_{\Omega_{1}} G_{1} C_{12} \gamma_{n, 2} \nabla \Phi_{22} \cdot \overline{\boldsymbol{u}_{1}} d \Omega_{1}= \\
=\rho_{2} \int_{\Gamma_{1}} C_{11} \gamma_{n, 1} \boldsymbol{u}_{1} \cdot \overline{\gamma_{n, 1} \boldsymbol{u}_{1}} d \Gamma_{1}+\rho_{2} \int_{\Gamma_{1}} C_{12} \gamma_{n, 1} \nabla \Phi_{22} \cdot \overline{\gamma_{n, 1} \boldsymbol{u}_{1}} d \Gamma_{1}= \\
=\rho_{2} \int_{\Gamma_{1}} \theta_{1} \Phi_{21} \cdot \overline{\left(-\gamma_{n, 1} \boldsymbol{u}_{1}\right)} d \Gamma_{1}+\rho_{2} \int_{\Gamma_{1}} \theta_{1} \Phi_{22} \cdot \overline{\left(-\gamma_{n, 1} \boldsymbol{u}_{1}\right)} d \Gamma_{1}= \\
=\rho_{2} \int_{\Gamma_{1}} \Phi_{21} \cdot \overline{-\frac{\partial \Phi_{21}}{\partial n_{2}}} d \Gamma_{1}+\rho_{2} \int_{\Gamma_{1}} \Phi_{22} \cdot-\frac{\partial \Phi_{21}}{\partial n_{2}} d \Gamma_{1}=\rho_{2} \int_{\Gamma_{1}}\left(\Phi_{21}+\Phi_{22}\right) \cdot-\frac{\partial \Phi_{21}}{\partial n_{2}} d \Gamma_{1} .
\end{aligned}
$$

Аналогично с учетом свойств операторов $C_{21}$ и $C_{22}, G_{2}$ преобразуем пятое и шестое слагаемые; получим

$$
\rho_{2} \int_{\Omega_{2}} G_{2} C_{21} \gamma_{n, 1} \boldsymbol{u}_{1} \cdot \overline{\nabla \Phi_{22}} d \Omega_{2}+\rho_{2} \int_{\Omega_{2}} G_{2} C_{22} \gamma_{n, 2} \nabla \Phi_{22} \cdot \overline{\nabla \Phi_{22}} d \Omega_{2}=\rho_{2} \int_{\Gamma_{2}}\left(\Phi_{21}+\Phi_{22}\right) \cdot \frac{\overline{\partial \Phi_{22}}}{\partial n_{2}} d \Gamma_{2} .
$$

Кроме того, используя вспомогательную задачу III, преобразуем седьмое слагаемое в (2.3.11):

$$
\rho_{3} \int_{\Omega_{2}} G_{2} C_{3} \gamma_{n, 2} \boldsymbol{u}_{2} \cdot \overline{\nabla \Phi_{22}} d \Omega_{2}=\rho_{3} \int_{\Gamma_{2}} \Phi_{3} \cdot \overline{\left(-\frac{\partial \Phi_{3}}{\partial n_{2}}\right)} d \Gamma_{2} .
$$


Тогда взамен (2.3.11) получим

$$
\begin{array}{r}
(\mathcal{T} z, z)_{\mathcal{H}}=\rho_{1} \int_{\Omega_{1}}\left|\boldsymbol{u}_{1}\right|^{2} d \Omega_{1}+\sum_{r=1}^{m} \int_{\Omega_{1}}\left|\boldsymbol{y}_{r}\right|^{2} d \Omega_{1}+\rho_{2} \int_{\Omega_{2}}\left|\nabla \Phi_{2}\right|^{2} d \Omega_{2}+\rho_{3} \int_{\Omega_{3}}\left|\nabla \Phi_{3}\right|^{2} d \Omega_{3}+ \\
+g\left(\rho_{2}-\rho_{1}\right) \int_{\Gamma_{1}}\left|\zeta_{1}\right|^{2} d \Gamma_{1}+g\left(\rho_{3}-\rho_{2}\right) \int_{\Gamma_{2}}\left|\zeta_{2}\right|^{2} d \Gamma_{2}>0 .
\end{array}
$$

С учетом ограниченности оператора $\mathcal{T}$ из $(2.3 .12)$ можно установить, что он самосопряжен и положительно определен.

Изучим теперь общие свойства оператора $\mathcal{A}$ из (2.3.7), (2.3.9).

Лемма 2.4. Операторная матрица $\mathcal{A}$ допускает факторизацию в виде произведения трех матрии, с симметричным окаймлением средней матриць:

$$
\mathcal{A}=\left(\begin{array}{cc}
\tilde{\mathcal{A}} & 0 \\
0 & \hat{I}_{2}
\end{array}\right)\left(\begin{array}{cc}
\tilde{I} & \tilde{\mathcal{G}}^{+} \\
-\tilde{\mathcal{G}} & \mathcal{O}
\end{array}\right)\left(\begin{array}{cc}
\tilde{\mathcal{A}} & 0 \\
0 & \hat{I}_{2}
\end{array}\right)
$$

где

$$
\begin{gathered}
\tilde{\mathcal{G}}^{+}:=\left(\begin{array}{cc}
g\left(\rho_{2}-\rho_{1}\right) A^{-1 / 2} G_{1} & 0 \\
\hat{0} & \hat{0} \\
0 & g\left(\rho_{3}-\rho_{2}\right) G_{2}
\end{array}\right), \quad \tilde{\mathcal{G}}:=\left(\begin{array}{ccc}
g\left(\rho_{2}-\rho_{1}\right) G_{1}^{*} A^{-1 / 2} & 0 & 0 \\
0 & 0 & \\
0 & 0 & g\left(\rho_{3}-\rho_{2}\right) G_{2}^{*}
\end{array}\right), \\
\tilde{\mathcal{A}}:=\left(\begin{array}{ccc}
A^{1 / 2} & \hat{0}^{\tau} & 0 \\
\hat{0} & \hat{I}_{m} & \hat{0} \\
0 & \hat{0}^{\tau} & I
\end{array}\right), \quad \tilde{I}:=\left(\begin{array}{ccc}
I & \hat{\alpha}^{\tau} & 0 \\
-\hat{\alpha} & \hat{\beta} & 0 \\
0 & 0 & 0
\end{array}\right), \quad \hat{I}_{2}:=\operatorname{diag}\{I, I\} .
\end{gathered}
$$

Лемма 2.5. Имеет место соотношение

$$
A^{-1 / 2} G_{1}=\left.\left(G_{1}^{*} A^{-1 / 2}\right)^{*}\right|_{\mathcal{D}\left(G_{1}\right)},
$$

причем замыкание по непрерывности оператора $A^{-1 / 2} G_{1}$ совпадает с $\left(G_{1}^{*} A^{-1 / 2}\right)^{*}$.

Доказательство аналогично доказательству леммы 1.8.

Из леммы 2.5 непосредственно получаем следующий результат.

Лемма 2.6. Справедливо соотношение $\overline{\tilde{\mathcal{G}}^{+}}=\tilde{\mathcal{G}}^{*}, \tilde{\mathcal{G}}^{+}=\left.\tilde{\mathcal{G}}^{*}\right|_{\mathcal{D}\left(G_{1}\right) \oplus \mathcal{D}\left(G_{2}\right)}$.

Лемма 2.7. Операторная матрица $\mathcal{A}$ из (2.3.7)-(2.3.9) является аккретивной в пространcmве $\mathcal{H}$, m.e.

$$
\operatorname{Re}(\mathcal{A} z, z)_{\mathcal{H}} \geqslant 0 \quad \forall z \in \mathcal{D}(\mathcal{A}) \subset \mathcal{H} .
$$

Доказательство. Действительно,

$$
\begin{aligned}
& (\mathcal{A} z, z)_{\mathcal{H}}=\left(A \boldsymbol{u}_{1}, \boldsymbol{u}_{1}\right)_{\boldsymbol{J}_{0, S_{1}}\left(\Omega_{1}\right)}+\left(\hat{\alpha}^{\tau} \hat{y}, \boldsymbol{u}_{1}\right)_{\hat{\boldsymbol{J}}_{0, S_{1}}\left(\Omega_{1}\right)}+b_{1}\left(G_{1} \zeta_{1}, \boldsymbol{u}_{1}\right)_{\boldsymbol{J}_{0, S_{1}}\left(\Omega_{1}\right)}-\left(\hat{\alpha} \boldsymbol{u}_{1}, \hat{y}\right)_{\hat{\boldsymbol{J}}_{0, S_{1}}\left(\Omega_{1}\right)}+ \\
& +(\hat{\beta} \hat{y}, \hat{y})_{\hat{\boldsymbol{J}}_{0, S_{1}}\left(\Omega_{1}\right)}+b_{2}\left(G_{2} \zeta_{2}, \nabla \Phi_{22}\right)_{\boldsymbol{G}_{h, S_{2}}\left(\Omega_{2}\right)}-b_{1}\left(\gamma_{n, 1} \boldsymbol{u}_{1}, \zeta_{1}\right)_{L_{2, \Gamma_{1}}}-b_{2}\left(\gamma_{n, 2} \nabla \Phi_{22}, \zeta_{2}\right)_{L_{2, \Gamma_{2}}}=
\end{aligned}
$$

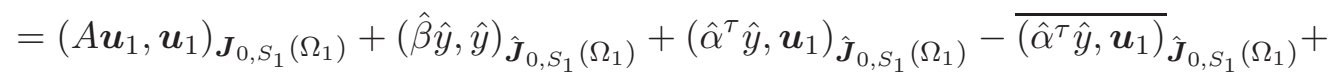

$$
\begin{aligned}
& +b_{1}\left(\zeta_{1}, \gamma_{n, 1} \boldsymbol{u}_{1}\right)_{L_{2, \Gamma_{1}}}-b_{1}{\overline{\left(\zeta_{1}, \gamma_{n, 1} \boldsymbol{u}_{1}\right)}}_{L_{2, \Gamma_{1}}}+b_{2}\left(\zeta_{2}, \gamma_{n, 2} \nabla \Phi_{22}\right)_{L_{2, \Gamma_{2}}}-b_{2}{\overline{\left(\zeta_{2}, \gamma_{n, 2} \nabla \Phi_{22}\right)_{L_{2, \Gamma_{2}}}}},
\end{aligned}
$$

откуда следует, что

$$
\operatorname{Re}(\mathcal{A} z, z)_{\mathcal{H}}=\left(A \boldsymbol{u}_{1}, \boldsymbol{u}_{1}\right)_{\boldsymbol{J}_{0, S_{1}}\left(\Omega_{1}\right)}+\sum_{r=1}^{m} \beta_{r}\left(y_{r}, y_{r}\right)_{\boldsymbol{J}_{0, S_{1}}\left(\Omega_{1}\right)} \geqslant 0 .
$$

Введем операторную матрицу

$$
\mathcal{J}_{a}:=\mathcal{J}_{0}+a \operatorname{diag}\left(0 ; \hat{0}^{\tau} ; I ; I ; I\right), \quad a>0, \quad \mathcal{J}_{0}:=\left(\begin{array}{cc}
\tilde{I} & \tilde{\mathcal{G}} \\
-\tilde{\mathcal{G}}^{*} & \mathcal{O}
\end{array}\right) .
$$


Тогда для $\mathcal{J}_{a}$ выполняется неравенство

$$
\operatorname{Re}\left(\mathcal{J}_{a} z, z\right)_{\mathcal{H}} \geqslant c\|z\|_{\mathcal{H}}^{2}, \quad c>0 .
$$

Из (2.3.14) следует, что операторная матрица $\mathcal{A}$ из (2.3.13) принимает вид

$$
\mathcal{A}=\operatorname{diag}\left(\tilde{\mathcal{A}} ; \hat{I}_{2}\right) \mathcal{J}_{a} \operatorname{diag}\left(\tilde{\mathcal{A}} ; \hat{I}_{2}\right)-a \operatorname{diag}\left(0 ; \hat{0}^{\tau} ; I ; I ; I\right)=: \mathcal{A}_{a}-a \operatorname{diag}\left(0 ; \hat{0}^{\tau} ; I ; I ; I\right) .
$$

При этом оператор $\mathcal{A}_{a}$ представлен в виде произведения трех сомножителей, каждый из которых имеет ограниченный обратный. Поэтому $\mathcal{A}_{a}$ допускает расширение путем замыкания среднего сомножителя, и в итоге возникает максимальный равномерно аккретивный оператор.

Лемма 2.8. Замыкание $\overline{\mathcal{A}}_{a}$ оператора $\mathcal{A}_{a}$ представляется в виде

$$
\overline{\mathcal{A}}_{a}=\operatorname{diag}\left(A^{1 / 2} ; \hat{I} ; I\right) \overline{\mathcal{J}}_{a} \operatorname{diag}\left(A^{1 / 2} ; \hat{I} ; I\right),
$$

где

$$
\overline{\mathcal{J}}_{a}=\left(\begin{array}{cc}
\tilde{I}_{a} & \tilde{\mathcal{G}}^{*} \\
-\tilde{\mathcal{G}} & a \hat{I}_{2}
\end{array}\right), \quad \tilde{I}_{a}:=\tilde{I}+a \operatorname{diag}\{0 ; \hat{0} ; I\}
$$

$\overline{\mathcal{J}}_{a}$ - равномерно аккретивный оператор, для которого выполнено свойство (2.3.15) (с заменой $\left.\mathcal{J}_{a} \rightarrow \overline{\mathcal{J}}_{a}\right)$. При этом

$$
\begin{aligned}
& \mathcal{D}\left(\overline{\mathcal{A}}_{a}\right)=\left\{y=\left(\boldsymbol{u}_{1} ; \hat{y} ; \nabla \Phi_{22} ; \zeta_{1} ; \zeta_{2}\right)^{\tau}: \boldsymbol{u}_{1} \in \mathcal{D}\left(A^{1 / 2}\right),\right. \\
&\left.A^{1 / 2} \boldsymbol{u}_{1}+\sum_{r=1}^{m} \alpha_{r}^{1 / 2} \boldsymbol{y}_{r}+g\left(\rho_{1}-\rho_{2}\right)\left(G_{1}^{*} A^{-1 / 2}\right)^{*} \zeta_{1} \in \mathcal{D}\left(A^{1 / 2}\right)\right\}, \quad \mathcal{R}\left(\overline{\mathcal{A}}_{a}\right)=\mathcal{H},
\end{aligned}
$$

и оператор $\overline{\mathcal{A}}_{a}$ действует на $\mathcal{D}\left(\overline{\mathcal{A}}_{a}\right)$ по закону

$$
\overline{\mathcal{A}}_{a} z=\left(\begin{array}{c}
A^{1 / 2}\left(A^{1 / 2} \boldsymbol{u}_{1}+\hat{\alpha}^{\tau} \hat{y}+g\left(\rho_{1}-\rho_{2}\right)\left(G_{1}^{*} A^{-1 / 2}\right)^{*} \zeta_{1}\right) \\
-\hat{\alpha} A^{1 / 2} \boldsymbol{u}_{1}+\hat{\beta} \hat{y} \\
a \nabla \Phi_{22}+g\left(\rho_{2}-\rho_{3}\right) G_{2} \zeta_{2} \\
-g\left(\rho_{1}-\rho_{2}\right) G_{1}^{*} \boldsymbol{u}_{1}+a \zeta_{1} \\
-g\left(\rho_{2}-\rho_{3}\right) G_{2}^{*} \nabla \Phi_{22}+a \zeta_{2}
\end{array}\right)
$$

2.4. О разрешимости начально-краевой задачи. Формулировка спектральной проблемы.

Теорема о сильной разрешимости исходной началъно-краевой задачи. Вернемся к задаче (2.3.7)-(2.3.9) и перепишем ее с учетом (2.3.16) в виде

$$
\begin{gathered}
\mathcal{T} \frac{d z}{d t}=-\left(\mathcal{A}_{a}-a \mathcal{P}\right) z+g(t), \quad z(0)=z^{0}:=\left(\boldsymbol{u}_{1}^{0} ; \hat{0} ; \boldsymbol{u}_{2}^{0} ; \zeta_{1}^{0} ; \zeta_{2}^{0}\right)^{\tau}, \\
z=\left(\boldsymbol{u}_{1} ; \hat{y} ; \nabla \Phi_{22} ; \zeta_{1} ; \zeta_{2}\right)^{\tau}, \quad \mathcal{P}:=\operatorname{diag}\left(0 ; \hat{0}^{\tau} ; I ; I ; I\right) .
\end{gathered}
$$

Рассмотрим также аналогичную задачу с замкнутым максимальным аккретивным оператором:

$$
\mathcal{T} \frac{d z}{d t}=-\left(\overline{\mathcal{A}}_{a}-a \mathcal{P}\right) z+g(t), \quad z(0)=z^{0} .
$$

Оператор $\mathcal{T}$ самосопряженный, положительно определенный и ограниченный в $\mathcal{H}$; значит, для него существует обратный оператор $\mathcal{T}^{-1}$, обладающий теми же свойствами. Тогда из (2.4.1) имеем:

$$
\frac{d z}{d t}=-\mathcal{T}^{-1}\left(\overline{\mathcal{A}}_{a}-a \mathcal{P}\right) z+\mathcal{T}^{-1} g(t), \quad z(0)=z^{0}
$$

Введем в $\mathcal{H}$ эквивалентную норму по формуле

$$
\langle z, z\rangle:=(\mathcal{T} z, z)_{\mathcal{H}}=\left(\mathcal{T}^{1 / 2} z, \mathcal{T}^{1 / 2} z\right)_{\mathcal{H}}
$$

Эквивалентность этой нормы стандартной норме следует из свойств оператора $\mathcal{T}$. Легко проверить, что оператор $\mathcal{T}^{-1}\left(\overline{\mathcal{A}}_{a}-a \mathcal{P}\right)$ будет максимальным аккретивным в новом скалярном произведении, и тогда $-\mathcal{T}^{-1}\left(\overline{\mathcal{A}}_{a}-a \mathcal{P}\right)$ является генератором сжимающей $C_{0}$-полугруппы. Поэтому по 
теореме Филлипса (см. [12, с.166]) задача Коши (2.4.2) имеет единственное сильное решение на отрезке $[0, T]$, если выполнены следующие условия:

$$
z^{0} \in \mathcal{D}(\mathcal{A}) \subset \mathcal{D}\left(\overline{\mathcal{A}}_{a}\right), \quad g(t) \in C^{1}([0, T] ; \mathcal{H}) .
$$

Из условий (2.4.3) получим соответствующие условия на начальные данные исходной задачи $(2.1 .2)-(2.1 .11)$.

Так, из принадлежности элемента $z^{0}$ области определения оператора $\mathcal{A}$ следует, что

$$
\boldsymbol{u}_{1}^{0} \in \mathcal{D}(A) \subset \mathcal{D}\left(A^{1 / 2}\right), \quad \zeta_{j}^{0} \in \mathcal{D}\left(G_{j}\right)=H_{\Gamma_{j}}^{1 / 2}, \quad j=1,2 .
$$

Аналогично получим условие для $\boldsymbol{f}_{k}(t)$ :

$$
\boldsymbol{f}_{k}(t) \in C^{1}\left([0, T] ; \boldsymbol{L}_{2}\left(\Omega_{k}\right)\right), \quad k=\overline{1,3} .
$$

Определение 2.1. Будем говорить, что исходная начально-краевая задача (2.1.2)-(2.1.11) имеет сильное решение $\left\{\boldsymbol{u}_{1}(t) ; \boldsymbol{u}_{2}(t) ; \boldsymbol{u}_{3}(t) ; \zeta_{1}(t) ; \zeta_{2}(t)\right\}$ на отрезке $[0, T]$, если выполнены следующие условия:

(1) $\boldsymbol{u}_{k}(t) \in C^{1}\left([0, T] ; \boldsymbol{J}_{0, S}\left(\Omega_{k}\right)\right), k=\overline{1,3}$;

(2) $\boldsymbol{v}_{1}(t)=I_{0,1}(t) \boldsymbol{u}_{1}(t)$ (см. (2.1.3)) обладает свойством $\boldsymbol{v}_{1}(t) \in C([0, T] ; \mathcal{D}(A))$;

(3) $\zeta_{j}(t) \in C^{1}\left([0, T] ; H_{\Gamma_{j}}^{1 / 2}\right), j=1,2$;

(4) для любого $t \in[0, T]$ выполнена система уравнений $(2.2 .25)-(2.2 .27)$, где все слагаемые в первом уравнении - элементы из $C\left([0, T] ; \boldsymbol{J}_{0, S_{1}}\left(\Omega_{1}\right)\right)$, во втором - элементы из $C\left([0, T] ; \boldsymbol{J}_{0, S_{2}}\left(\Omega_{2}\right)\right)$, а в третьем и четвертом - из $C\left([0, T] ; H_{\Gamma_{1}}^{1 / 2}\right)$ и $C\left([0, T] ; H_{\Gamma_{2}}^{1 / 2}\right)$ соответственно;

(5) выполнены начальные условия (2.2.28).

Сформулируем теперь теорему существования и единственности сильного решения задачи $(2.1 .2)-(2.1 .11)$.

Теорема 2.2. Пусть выполнены условия

$$
\begin{gathered}
\boldsymbol{u}_{1}^{0} \in \mathcal{D}(A) \subset \boldsymbol{J}_{0, S_{1}}^{1}\left(\Omega_{1}\right), \quad \boldsymbol{u}_{l} \in \boldsymbol{H}^{1}\left(\Omega_{l}\right) \cap \boldsymbol{J}_{0, S_{l}}\left(\Omega_{l}\right), \quad \zeta_{j}^{0} \in H_{\Gamma_{j}}^{1 / 2}, \quad j=1,2, \quad l=2,3 ; \\
\boldsymbol{f}_{k}(t) \in C^{1}\left([0, T] ; \boldsymbol{L}_{2}\left(\Omega_{k}\right)\right), \quad k=\overline{1,3} .
\end{gathered}
$$

Тогда начально-краевая задача (2.1.2)-(2.1.11) имеет единственное сильное решение на [0;T].

Доказательство, основанное на обратном переходе от задачи Коши (2.4.2) к начально-краевой задаче (2.1.2)-(2.1.11) с использованием результатов лемм 2.2, 2.3-2.8, проводится по схеме доказательства теорем 2, 3 и 5 работы [9].

К задаче о нормалъных колебаниях гидросистемы. Рассмотрим теперь постановку задачи о малых нормальных движениях исследуемой гидросистемы, т.е. о таких решениях однородной задачи (2.4.1) с замкнутым основным оператором, которые зависят от $t$ по закону

$$
z(t):=\left(\boldsymbol{u}_{1}(t) ; \hat{y}(t) ; \nabla \Phi_{22}(t) ; \zeta_{1}(t) ; \zeta_{2}(t)\right)^{\tau}=\left(\boldsymbol{u}_{1} ; \hat{y} ; \nabla \Phi_{22} ; \zeta_{1} ; \zeta_{2}\right)^{\tau} e^{-\lambda t},
$$

где $\lambda \in \mathbb{C}$ - комплексный декремент затухания, а $\left(\boldsymbol{u}_{1} ; \hat{y} ; \nabla \Phi_{22} ; \zeta_{1} ; \zeta_{2}\right)^{\tau}$ - амплитудный элемент. Тогда для отыскания амплитудных элементов возникает спектральная задача

$$
\begin{gathered}
A^{1 / 2}\left(A^{1 / 2} \boldsymbol{u}_{1}+\hat{\alpha}^{\tau} \hat{y}+g\left(\rho_{1}-\rho_{2}\right)\left(G_{1}^{*} A^{-1 / 2}\right)^{*} \zeta_{1}\right)=\lambda\left(\left(\rho_{1} I+\rho_{2} G_{1} C_{11} \gamma_{n, 1}\right) \boldsymbol{u}_{1}+\rho_{2} G_{1} C_{12} \gamma_{n, 2} \nabla \Phi_{22}\right), \\
-\alpha_{r}^{1 / 2} A^{1 / 2} \boldsymbol{u}_{1}+\beta_{r} \boldsymbol{y}_{r}=\lambda \boldsymbol{y}_{r}, \quad r=\overline{1, m}, \\
g\left(\rho_{2}-\rho_{3}\right) G_{2} \zeta_{2}=\lambda\left(\rho_{2} G_{2} C_{21} \gamma_{n, 1} \boldsymbol{u}_{1}+\left(\rho_{2} G_{2} C_{22} \gamma_{n, 2}+\rho_{3} G_{2} C_{3} \gamma_{n, 2}\right) \nabla \Phi_{22}\right), \\
-G_{1}^{*} \boldsymbol{u}_{1}=\lambda \zeta_{1}, \quad-G_{2}^{*} \nabla \Phi_{22}=\lambda \zeta_{2} .
\end{gathered}
$$


В случае $\lambda=0$ приходим к соотношениям

$$
\begin{gathered}
A^{1 / 2}\left(A^{1 / 2} \boldsymbol{u}_{1}+\hat{\alpha}^{\tau} \hat{y}+g\left(\rho_{1}-\rho_{2}\right)\left(G_{1}^{*} A^{-1 / 2}\right)^{*} \zeta_{1}\right)=\mathbf{0}, \\
\beta_{r} \boldsymbol{y}_{r}=\alpha_{r}^{1 / 2} A^{1 / 2} \boldsymbol{u}_{1}, \quad r=\overline{1, m}, \quad G_{2} \zeta_{2}=0, \quad G_{1}^{*} \boldsymbol{u}_{1}=\mathbf{0}, \quad G_{2}^{*} \nabla \Phi_{22}=\mathbf{0} .
\end{gathered}
$$

Домножив скалярно третье соотношение на элемент $\boldsymbol{u}_{2} \in \boldsymbol{J}_{0, S_{2}}\left(\Omega_{2}\right)$, будем иметь

$$
0=\left(G_{2} \zeta_{2}, \boldsymbol{u}_{2}\right)_{\boldsymbol{L}_{2}\left(\Omega_{2}\right)}=\left\langle\zeta_{2}, \gamma_{n, 2} \boldsymbol{u}_{2}\right\rangle_{L_{2, \Gamma_{2}}}
$$

откуда в силу произвольности $\boldsymbol{u}_{2}$ следует, что $\zeta_{2}=0$. Аналогично из последних двух связей получаем, что $\boldsymbol{u}_{1}=\mathbf{0}, \nabla \Phi_{22}=\mathbf{0}$; тогда из второго соотношения следует, что $\boldsymbol{y}_{r}=\mathbf{0}, r=\overline{1, m}$, и, наконец, из первого -

$$
A^{1 / 2}\left(g\left(\rho_{1}-\rho_{2}\right)\left(G_{1}^{*} A^{-1 / 2}\right)^{*} \zeta_{1}\right)=\mathbf{0} .
$$

Домножая это соотношение на $\left(g\left(\rho_{1}-\rho_{2}\right)\right)^{-1}$, а затем скалярно на элемент $\boldsymbol{u}_{1} \in \boldsymbol{J}_{0, S_{1}}\left(\Omega_{1}\right)$, получим

$$
0=\left(A^{1 / 2}\left(G_{1}^{*} A^{-1 / 2}\right)^{*} \zeta_{1}, \boldsymbol{u}_{1}\right)_{\boldsymbol{L}_{2}\left(\Omega_{1}\right)}=\left\langle\zeta_{1}, G_{1}^{*} A^{-1 / 2} A^{1 / 2} \boldsymbol{u}_{1}\right\rangle_{L_{2, \Gamma_{1}}}=\left\langle\zeta_{1}, \gamma_{n, 1} \boldsymbol{u}_{1}\right\rangle_{L_{2, \Gamma_{1}}} .
$$

Отсюда можем заключить, что $\zeta_{1}=0$ в силу произвольности элемента $\boldsymbol{u}_{1}$.

Таким образом, задача (2.4.5) имеет лишь тривиальное решение, т.е. $\lambda=0$ не является собственным значением задачи (2.4.4).

Рассмотрим еще случай, когда $\lambda=\beta_{s}, s=\overline{1, m}$. Вместо (2.4.4) теперь будем иметь систему уравнений

$$
\begin{gathered}
A^{1 / 2}\left(A^{1 / 2} \boldsymbol{u}_{1}+\hat{\alpha}^{\tau} \hat{y}+g\left(\rho_{1}-\rho_{2}\right)\left(G_{1}^{*} A^{-1 / 2}\right)^{*} \zeta_{1}\right)=\beta_{s}\left(\left(\rho_{1} I+\rho_{2} G_{1} C_{11} \gamma_{n, 1}\right) \boldsymbol{u}_{1}+\rho_{2} G_{1} C_{12} \gamma_{n, 2} \nabla \Phi_{22}\right) \\
-\alpha_{r}^{1 / 2} A^{1 / 2} \boldsymbol{u}_{1}+\beta_{r} \boldsymbol{y}_{r}=\beta_{s} \boldsymbol{y}_{r}, \quad r=\overline{1, m} \\
g\left(\rho_{2}-\rho_{3}\right) G_{2} \zeta_{2}=\beta_{s}\left(\rho_{2} G_{2} C_{21} \gamma_{n, 1} \boldsymbol{u}_{1}+\left(\rho_{2} G_{2} C_{22} \gamma_{n, 2}+\rho_{3} G_{2} C_{3} \gamma_{n, 2}\right) \nabla \Phi_{22}\right) \\
G_{1}^{*} \boldsymbol{u}_{1}=\beta_{s} \zeta_{1}, \quad G_{2}^{*} \nabla \Phi_{22}=\beta_{s} \zeta_{2} .
\end{gathered}
$$

Из второго соотношения при $r=s$ заключаем, что $\boldsymbol{u}_{1}=\mathbf{0}$ в силу обратимости оператора $A^{1 / 2}$. Тогда из четвертого уравнения получим, что $\zeta_{1}=0$, а из второго при $r \neq s-$ свойство $\boldsymbol{y}_{r}=\mathbf{0}$. Выразим из последнего соотношения $\zeta_{2}$ и подставим в третье. Получим

$$
\left(g\left(\rho_{2}-\rho_{3}\right) \beta_{s}^{-1} G_{2} G_{2}^{*}-\beta_{s}\left(\rho_{2} G_{2} C_{22} \gamma_{n, 2}+\rho_{3} G_{2} C_{3} \gamma_{n, 2}\right)\right) \nabla \Phi_{22}=\mathbf{0} .
$$

Домножив (2.4.6) скалярно на элемент $\nabla \Phi_{22} \in \boldsymbol{G}_{h, S_{2}, \Gamma_{2}}\left(\Omega_{2}\right)$, получим

$$
\begin{aligned}
0=g\left(\rho_{2}-\rho_{3}\right) \beta_{s}^{-1} & \left(G_{2} G_{2}^{*} \nabla \Phi_{22}, \nabla \Phi_{22}\right)_{\boldsymbol{L}_{2}\left(\Omega_{2}\right)}- \\
& -\beta_{s} \rho_{2}\left(G_{2} C_{22} \gamma_{n, 2} \nabla \Phi_{22}, \nabla \Phi_{22}\right)_{\boldsymbol{L}_{2}\left(\Omega_{2}\right)}-\beta_{s} \rho_{3}\left(G_{2} C_{3} \gamma_{n, 2} \nabla \Phi_{22}, \nabla \Phi_{22}\right)_{\boldsymbol{L}_{2}\left(\Omega_{2}\right)} .
\end{aligned}
$$

Используя свойства операторов $C_{22}, C_{3}$ и $G_{2}$ для каждого из слагаемых, получим следующие соотношения:

$$
\begin{gathered}
\int_{\Omega_{2}} G_{2} G_{2}^{*} \nabla \Phi_{22} \cdot \overline{\nabla \Phi_{22}} d \Omega_{2}=\int_{\Gamma_{2}} \gamma_{n, 2} \nabla \Phi_{22} \cdot \overline{\gamma_{n, 2} \nabla \Phi_{22}} d \Gamma_{2}=\int_{\Gamma_{2}}\left|\frac{\partial \Phi_{22}}{\partial n}\right|^{2} d \Gamma_{2}, \\
\int_{\Omega_{2}} G_{2} C_{22} \gamma_{n, 2} \nabla \Phi_{22} \cdot \overline{\nabla \Phi_{22}} d \Omega_{2}=\int_{\Gamma_{2}} C_{22} \gamma_{n, 2} \nabla \Phi_{22} \cdot \overline{\gamma_{n, 2} \nabla \Phi_{22}} d \Gamma_{2}= \\
=-\int_{\Gamma_{2}} \theta_{2} \Phi_{22} \cdot \overline{-\frac{\partial \Phi_{22}}{\partial n}} d \Gamma_{2}=-\int_{\Omega_{2}}\left|\nabla \Phi_{22}\right|^{2} d \Omega_{2}, \\
\int_{\Omega_{2}} G_{2} C_{3} \gamma_{n, 2} \nabla \Phi_{22} \cdot \nabla \Phi_{22} d \Omega_{2}=\int_{\Gamma_{2}} C_{3} \gamma_{n, 2} \nabla \Phi_{22} \cdot \overline{\gamma_{n, 2} \nabla \Phi_{22}} d \Gamma_{2}=
\end{gathered}
$$




$$
=-\int_{\Gamma_{2}} \theta_{2} \Phi_{3} \cdot \overline{\frac{\partial \Phi_{22}}{\partial n_{2}}} d \Gamma_{2}=-\int_{\Gamma_{2}} \theta_{2} \Phi_{3} \cdot \overline{-\frac{\partial \Phi_{3}}{\partial n_{2}}} d \Gamma_{2}=-\int_{\Omega_{3}}\left|\nabla \Phi_{3}\right|^{2} d \Omega_{3}
$$

Подставляя эти результаты в (2.4.7), находим

$$
0=g\left(\rho_{2}-\rho_{3}\right) \beta_{s}^{-1} \int_{\Gamma_{2}}\left|\frac{\partial \Phi_{22}}{\partial n}\right|^{2} d \Gamma_{2}+\beta_{s} \rho_{2} \int_{\Omega_{2}}\left|\nabla \Phi_{22}\right|^{2} d \Omega_{2}+\beta_{s} \rho_{3} \int_{\Omega_{3}}\left|\nabla \Phi_{3}\right|^{2} d \Omega_{3},
$$

откуда следует, что $\nabla \Phi_{22}=\mathbf{0}$, а значит, $\zeta_{2}=0$. С учетом этих равенств из первого уравнения приходим к выводу, что $\boldsymbol{y}_{s}=\mathbf{0}$, т.е. $\lambda=\beta_{s}, s=\overline{1, m}$, тоже не является собственным значением задачи (2.4.4).

Опираясь на эти факты, преобразуем при $\lambda \neq 0, \lambda \neq \beta_{s}, s=\overline{1, m}$, задачу (2.4.4) к спектральной проблеме, исключив $\boldsymbol{y}_{r}$ и $\zeta_{j}, r=\overline{1, m}, j=1,2$. Имеем

$$
\begin{aligned}
\lambda\left(\begin{array}{cc}
\rho_{1} I+\rho_{2} G_{1} C_{11} \gamma_{n, 1} & \rho_{2} G_{1} C_{12} \gamma_{n, 2} \\
\rho_{2} G_{2} C_{21} \gamma_{n, 1} & \rho_{2} G_{2} C_{22} \gamma_{n, 2}+\rho_{3} G_{2} C_{3} \gamma_{n, 2}
\end{array}\right)\left(\begin{array}{c}
\boldsymbol{u}_{1} \\
\nabla \Phi_{22}
\end{array}\right)+ \\
+\frac{1}{\lambda}\left(\begin{array}{cc}
g\left(\rho_{1}-\rho_{2}\right) G_{1} G_{1}^{*} & 0 \\
0 & g\left(\rho_{2}-\rho_{3}\right) G_{2} G_{2}^{*}
\end{array}\right)\left(\begin{array}{c}
\boldsymbol{u}_{1} \\
\nabla \Phi_{22}
\end{array}\right)+ \\
+\left(\begin{array}{cc}
A_{1}\left(I-\sum_{r=1}^{m} \frac{\alpha_{r}}{\lambda-\beta_{r}}\right) & 0 \\
0 & 0
\end{array}\right)\left(\begin{array}{c}
\boldsymbol{u}_{1} \\
\nabla \Phi_{22}
\end{array}\right)=\left(\begin{array}{l}
\mathbf{0} \\
\mathbf{0}
\end{array}\right) .
\end{aligned}
$$

Осуществляя замену

$$
A^{1 / 2} \boldsymbol{u}_{1}=: \varphi \in \boldsymbol{J}_{0, S_{1}}\left(\Omega_{1}\right)
$$

приходим к спектральной проблеме для элемента $x:=\left(\varphi ; \nabla \Phi_{22}\right)^{\tau}$ :

$$
L(\lambda) x:=\left(\operatorname{diag}\left\{e_{0}(\lambda) ; 0\right\}-\lambda \operatorname{diag}\left\{A^{1 / 2} ; 0\right\} \tilde{\mathcal{C}} \operatorname{diag}\left\{A^{1 / 2} ; 0\right\}-\lambda^{-1} \operatorname{diag}\left\{b_{1} Q Q^{*} ; b_{2} G_{2} G_{2}^{*}\right\}\right) x=0,
$$

где

$$
e_{0}(\lambda):=1+\sum_{r=1}^{m} \alpha_{r}\left(\beta_{r}-\lambda\right)^{-1}, \quad \tilde{\mathcal{C}}:=\left(\begin{array}{cc}
\rho_{1} I+\rho_{2} G_{1} C_{11} \gamma_{n, 1} & \rho_{2} G_{1} C_{12} \gamma_{n, 2} \\
\rho_{2} G_{2} C_{21} \gamma_{n, 1} & \rho_{2} G_{2} C_{22} \gamma_{n, 2}+\rho_{3} G_{2} C_{3} \gamma_{n, 2}
\end{array}\right)
$$

в пространстве $\boldsymbol{J}_{0, S_{1}}\left(\Omega_{1}\right) \oplus \boldsymbol{J}_{0, S_{2}}\left(\Omega_{2}\right)$ для операторного пучка $L(\lambda)$.

Задачу (2.4.8) можно исследовать методами спектральной теории операторных пучков (см. [15]). В частности, можно установить, что спектр этой задачи дискретен, расположен в правой комплексной полуплоскости и состоит из шести ветвей собственных значений, которым отвечают пограничные волны на границе раздела $\Gamma_{1}$, поверхностные волны на $\Gamma_{2}$, внутренние диссипативные волны в области $\Omega_{1}$, а также диссипативные волны, обусловленные вязкоупругостью жидкости.

Подробно эти свойства решений спектральной задачи (2.4.8), а также свойства полноты и базисности ее корневых (собственных и присоединенных) элементов будут изучены в другой работе.

\section{СПИСОК ЛИТЕРАТУРЫ}

1. Агранович M. C. Спектральные задачи для сильно эллиптических систем второго порядка в областях с гладкой и негладкой границей// Усп. мат. наук. - 2002. - 57, № 5 (347). - С. 3-78.

2. Азизов Т. Я., Копачевский Н. Д., Орлова Л. Д. Эволюционная и спектральная задачи, порожденные проблемой малых движений вязкоупругой жидкости// Тр. СПб. мат. о-ва. - 1998. - 6. - С. 5-33.

3. Аскеров H. K., Крейн С. Г., Лаптев Г. И. Задача о колебаниях вязкой жидкости связанные с ней операторные уравнения// Функц. анал. прилож. - 1968. - 2, № 2. - С. 21-32.

4. Вронский Б. М. Нормальные колебания частично диссипативной гидросистемы// Динам. сист. - 2012. -2 (30), № 1-2. - С. 53-56.

5. Вронский Б. М. О малых движениях системы «жидкость-газ» в ограниченной области// Укр. мат. ж. - 2006. - 58, № 10. - C. 1326-1334. 
6. Гохберг Н. Ц., Крейн М. Г. Введение в теорию линейных несамосопряженных операторов в гильбертовом пространстве. - М.: Наука, 1965.

7. Келдыи М. В. О полноте собственных функций некоторых классов несамосопряженных линейных операторов// Усп. мат. наук. - 1971. - 26, № 4 (100). - С. 15-41.

8. Копачевский Н. Д. Абстрактная формула Грина и некоторые ее приложения. - Симферополь: Форма, 2016.

9. Копачевский Н. Д. О малых движениях системы из двух вязкоупругих жидкостей, заполняющих неподвижный сосуд// Динам. сист. - 2017. - 7 (35), № 1-2. - С. 109-145.

10. Копачевский Н. Д., Крейн С. Г., Нго Зуй Кан. Операторные методы в линейной гидродинамике: Эволюционные и спектральные задачи. - М.: Наука, 1989.

11. Копачевский H. Д., Семкина E. В. О малых движениях гидросистемы «вязкоупругая жидкостьидеальная жидкость», заполняющей неподвижный сосуд// Динам. сист. - 2017. - 7 (35), № 3. C. 207-228.

12. Крейн С. Г. Линейные дифференциальные уравнения в банаховом пространстве. - М.: Наука, 1967.

13. Крейн С. Г. О колебаниях вязкой жидкости в сосуде// Докл. АН СССР. - 1964. - 159, № 2. C. $262-265$.

14. Крейн С. Г., Лаптев Г. И. К задаче о движении вязкой жидкости в открытом сосуде// Функц. анал. прилож. - 1968. - 2, № 1. - С. 40-50.

15. Маркус A. С. Введение в спектральную теорию полиномиальных операторных пучков. - Кишинев: Штиинца, 1986.

16. Милославский A. И. Спектральный анализ малых колебаний вязкоупругой жидкости в открытом контейнере/ Деп. рукопись № 1221. - Киев: Ин-т мат. НАН Украины, 1989.

17. Милославский А. И. Спектр малых колебаний вязкоупругой жидкости в открытом сосуде// Усп. мат. наук. -1989 . -44 , № 4.

18. Милославский А. И. Спектр малых колебаний вязкоупругой наследственной среды// Докл. АН СССР. - 1989. - 309, № 3. - C. 532-536.

19. Agranovich $M$. Remarks on potential spaces and Besov spaces in a Lipschitz domain and on Whitney arrays on its boundary// Russ. J. Math. Phys. — 2008. — 15, № 2. — P. 146-155.

20. Eirich F. Rheology. Theory and Applications. — New York: Academic Press, 1956.

21. Gagliardo E. Caratterizzazioni delle tracce sulla frontiera relative ad alcune classi di funzioni in $n$ variabili// Rend. Sem. Mat. Univ. Padova. — 1957. — 27. — P. 284-305.

22. Kopachevsky N., Krein S. Operator Approach to Linear Problems of Hydrodynamics. Vol. 1. Self-Adjoint Problems for an Ideal Fluid. - Basel-Boston-Berlin: Birkhäuser, 2001.

23. Kopachevsky N., Krein S. Operator Approach to Linear Problems of Hydrodynamics. Vol. 2. Non-SelfAdjoint Problems for Viscous Fluids. - Basel-Boston-Berlin: Birkhäuser, 2003.

24. Kopachevsky N., Padula M., Vronsky B. M. Small motions and eigenoscillations of a system "fluid-gas" in a bounded region// Uchen. Zap. Tavrich. Nats. Univ. im. V. I. Vernadskogo. Ser. Mat. Mekh. Inform. Kibern. - 2007. - 20, № 1. - P. 3-55.

25. Miloslavskii A. Stability of a viscoelastic isotropic medium// Sov. Phys. Dokl. — 1985. - 33.

26. Miloslavsky A. Stability of certain classes of evolution equations// Sib. Math. J. — 1985. — 26, № 5. P. 723-735.

27. Rychkov $V$. On restrictions and extensions of the Besov and Triebel-Lizorkin spaces with respect to Lipschitz domains// J. London Math. Soc. - 1999. - 60, № 1. - P. 237-257.

Копачевский Николай Дмитриевич

Крымский федеральный университет им. В. И. Вернадского, Симферополь

E-mail: kopachevsky@list.ru

Семкина Екатерина Владимировна

Крымский федеральный университет им. В. И. Вернадского, Симферополь

E-mail: kozirno@email.com 